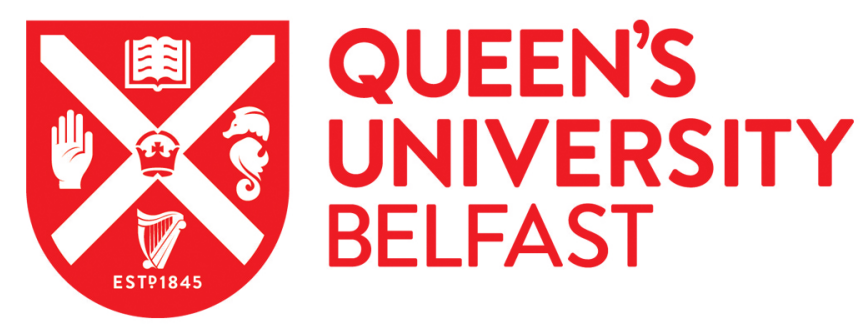

\title{
An overview and update on the epidemiology of flavonoid intake and cardiovascular disease risk: Flavonoid intake and cardiovascular disease risk
}

Parmenter, B. H., Croft, K. D., Hodgson, J. M., Dalgaard, F., Bondonno, C. P., Lewis, J. R., Cassidy, A., Scalbert, A., \& Bondonno, N. P. (2020). An overview and update on the epidemiology of flavonoid intake and cardiovascular disease risk: Flavonoid intake and cardiovascular disease risk. Food \& Function, 11(8), 67776806. https://doi.org/10.1039/DOFO01118E

Published in:

Food \& Function

Document Version:

Peer reviewed version

Queen's University Belfast - Research Portal:

Link to publication record in Queen's University Belfast Research Portal

Publisher rights

Copyright 2020 Royal Society of Chemistry. This work is made available online in accordance with the publisher's policies. Please refer to any applicable terms of use of the publisher.

\section{General rights}

Copyright for the publications made accessible via the Queen's University Belfast Research Portal is retained by the author(s) and / or other copyright owners and it is a condition of accessing these publications that users recognise and abide by the legal requirements associated with these rights.

\section{Take down policy}

The Research Portal is Queen's institutional repository that provides access to Queen's research output. Every effort has been made to ensure that content in the Research Portal does not infringe any person's rights, or applicable UK laws. If you discover content in the

Research Portal that you believe breaches copyright or violates any law, please contact openaccess@qub.ac.uk. 


\section{An overview and update on the epidemiology of flavonoid intake and cardiovascular disease risk}

Short title: Flavonoid intake and cardiovascular disease risk

Benjamin H. Parmenter, ${ }^{*}{ }^{*}$ Kevin D. Croft PhD,${ }^{1}$ Jonathan M. Hodgson PhD, ${ }^{2,4}$ Frederik

Dalgaard MD,${ }^{3}$ Catherine P. Bondonno PhD $, 2,4$ Joshua R. Lewis PhD, $, 2,4,5$ Aedín Cassidy

$\mathrm{PhD},{ }^{6}$ Augustin Scalbert PhD, ${ }^{7}$ Nicola P. Bondonno PhD,,${ }^{1,2,6}$

5 Research Foundation, Perth, Australia;

$6 \quad{ }^{2}$ School of Medical and Health Sciences, Edith Cowan University, Perth, Australia;

$7 \quad{ }^{3}$ Department of Cardiology, Herlev \& Gentofte University Hospital, Copenhagen, Denmark;

$8 \quad{ }^{4}$ Medical School, University of Western Australia, Perth, Australia;

$9{ }^{5}$ Centre for Kidney Research, School of Public Health, The University of Sydney, Sydney,

10 Australia;

$11{ }^{6}$ Institute for Global Food Security, Queen's University, Belfast, Northern Ireland;

$12{ }^{7}$ Biomarkers Group, International Agency for Research on Cancer, Lyon, France.

*Correspondence: Benjamin H Parmenter

16 Tel: 0473440405

17 Email: Benjamin.Parmenter@research.uwa.edu.au

Word count: 10,083; Total Tables: 4; Total Figures: 1

19 Key indexing terms: Flavonoids, epidemiology, cardiovascular disease, cerebrovascular

20 disease, stroke, ischemic heart disease, peripheral artery disease 
21 Acknowledgements: BHP is funded by an Australian Government Research Training Program

22 Scholarship. NPB is funded by a National Health and Medical Research Council Early Career

23 Fellowship (Grant number APP1159914), Australia. JRL is funded by a National Heart

24 Foundation Future Leader Fellowship (ID: 102817). The salary of JMH is supported by a

25 National Health and Medical Research Council of Australia Senior Research Fellowship (Grant

26 number APP1116937).

27 Conflicts of interest: The funding sources had no role in the design or writing of this

28 manuscript, or decision to submit the paper for publication; there are no conflicts of interest to

29 declare.

30 Disclaimer: Where authors are identified as personnel of the International Agency for

31 Research on Cancer / World Health Organization, the authors alone are responsible for the views expressed in this article and they do not necessarily represent the decisions, policy or views of the International Agency for Research on Cancer / World Health Organization. 


\section{Abstract}

There is an accumulating body of literature reporting on dietary flavonoid intake and the risk of cardiovascular disease (CVD) in prospective cohort studies. This makes apparent the need for an overview and update on the current state of the science. To date, at least 27 prospective cohorts (in 44 publications) have evaluated the association between estimated habitual flavonoid intake and CVD risk. At this time, the totality of evidence suggests long-term consumption of flavonoid-rich foods may be associated with a lower risk of fatal and non-fatal ischemic heart disease (IHD), cerebrovascular disease, and total CVD; disease outcomes which are principally, though not exclusively, composed of cases of atherosclerotic CVD (ASCVD).

44 To date, few studies have investigated outcome specific ASCVD, such as peripheral artery disease (PAD) or ischemic stroke. Of the flavonoid subclasses investigated, evidence more often implicates diets rich in anthocyanins, flavan-3-ols, and flavonols in lowering the risk of CVD. Although inferences are restricted by confounding and other inherent limitations of observational studies, causality appears possible based on biological plausibility, temporality, and the relative consistency of the reported associations. However, whether the associations observed represent a benefit of the isolated bioactives per se, or are a signal of the bioactives acting in concert with the co-occurring nutrient matrix within flavonoid-bearing foods, are issues of consideration. Thus, the simple interpretation, and the one most relevant for dietary advice, is that consumption of flavonoid-rich foods or diets higher in flavonoids, appear nutritionally beneficial in the prevention of CVD. 
59 Table of contents

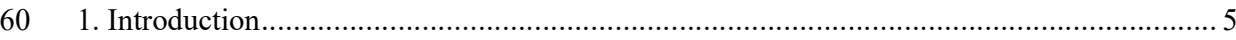

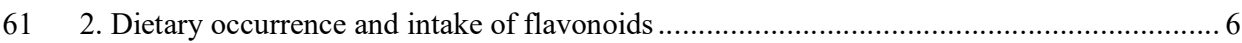

62 3. Calculation of flavonoid intake and how this has changed over the years ..................... 10

63 4. Biological mechanisms of flavonoids in cardiovascular disease............................... 15

64 5. Flavonoid intake and cardiovascular disease risk: epidemiological evidence.................17

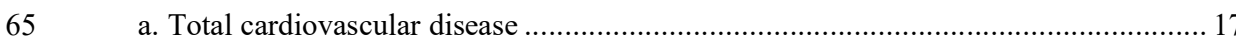

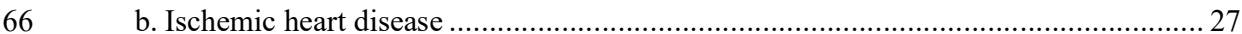

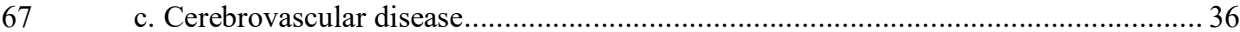

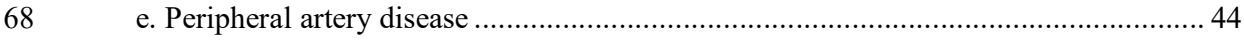

69 6. Discussion: current evidence —-future epidemiological frontiers.............................. 47

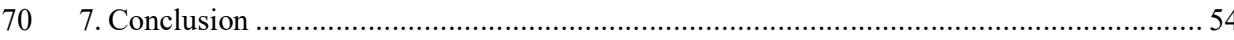

71

72

73

75

76

77

78

79

80 


\section{Introduction}

The 2017 Global Burden of Disease study observed that nutrition is a major contributor to cardiovascular morbidity and mortality, both in developed and developing countries. ${ }^{2}$ This highlights the need to shift global dietary trends for the health of humanity. ${ }^{1,2}$ Overall dietary patterns, generally higher in fruits, vegetables, wholegrains, nuts, seeds, legumes, seafood and yoghurt; moderate to modest in poultry, milk, cheese, eggs, alcohol and unprocessed red meat; and low to absent in processed meat, refined grains, and ultra-processed foods; have been identified as providing cardio-metabolic benefits, which translate into a lower risk of developing cardiovascular disease (CVD). ${ }^{3}$ The contribution of other regularly consumed items, such as tea and coffee, to cardio-metabolic health appears less certain. ${ }^{3}$ Attempts to determine the contribution of specific nutritive components, underpinning the benefits of such dietary patterns rich in plant foods, is underway. In addition to vitamins, minerals, and macronutrients, plant foods contain a wide variety of bioactive agents, such as (poly)phenols, terpenoids, and nitrogen containing compounds that might confer health benefits. ${ }^{4}$ Flavonoids, a class of (poly)phenolics, have gained specific interest as findings from epidemiological studies throughout the 1990's and 2000's indicate they may be responsible, at least in part, for the cardiovascular-protective benefits of diets rich in plant-foods.

In 1993, the first prospective study to evaluate the cardio-protective benefits of flavonoids observed that participants with the highest flavonoid intakes had a lower risk of ischemic heart disease (IHD) mortality. ${ }^{5}$ Since this time, a rapidly accumulating body of epidemiological evidence, supported by growing evidence from human intervention studies, has emerged. Comprehensively reviewed in 2005 by Arts and Hollman, ${ }^{6}$ as well as by Graf et al., ${ }^{7}$ and again in 2012 by Peterson et al., ${ }^{8}$ we have sought to provide an overview and update on the epidemiological evidence regarding the association between habitual flavonoid intake and CVD incidence and mortality in prospective cohorts. We begin by covering the dietary occurrence and intake of flavonoids. First, we describe the different flavonoid subclasses and 
their food sources before continuing with an overview of global consumption trends and intake differences between populations. The quantification of flavonoid intake follows, describing advances in composition databases, how these have changed over the years, and the potential impact this has for cohort studies. An overview of biological mechanisms, by which flavonoids mitigate CVD initiation and progression, precedes the synthesis of epidemiological evidence regarding the association of flavonoid intake with CVD risk. Here, we focus on total flavonoid intake and consumption of major flavonoid subclasses and their association with total CVD and CVD of atherosclerotic origin, before discussing major findings from outcome-specific prospective cohort studies on IHD, cerebrovascular disease and peripheral artery disease (PAD) spanning 1993 to March 2020. Following the update on the current state of the art, the discussion addresses broader issues of importance to flavonoid epidemiology research. We discuss the central hypothesis, its evidence and limitations, that flavonoid-rich dietary intake mediates intermediate endpoints of CVD, which modulates the trajectory of chronic CVD. Thereafter, we highlight critical gaps and needs for advancing flavonoid epidemiology, including the use of objective intake measures, cumulative measures of exposure, and the rationale for targeting ASCVD rather than all-cause CVD. Concluding the review is a summary of progress in the field over the past $\sim 25$ years and major future pathways for its continued development.

\section{Dietary occurrence and intake of flavonoids}

Flavonoids are the most common group of plant phenolics occurring ubiquitously throughout food plants and their products. ${ }^{9}$ To this end, a recent report estimates over 15,000 different flavonoids have been identified throughout the plant Kingdom, ${ }^{10}$ with at least several hundred occurring in edible components. ${ }^{11}$ In plants, flavonoids occur as secondary metabolites, which are not essential to the growth or life of the plant. ${ }^{12,13}$ Instead, flavonoids contribute to the protection of plants against pests and herbivores, as well as plant disease, season and climate, 
geography, and other local challenges, to increase the plant's overall ability to survive. ${ }^{12,13}$ Flavonoids, found in edible plants and their food products, are part of the human diet. ${ }^{13,14}$ They are not essential constituents in the classic nutrition sense; rather, they are non-nutrients, which can exert biologically relevant effects in vivo. ${ }^{13,14}$ Based on their underlying structure, flavonoids can be subdivided into six major subclasses, namely, the flavonols, flavones, flavanones, isoflavones, flavan-3-ols, and anthocyanins. ${ }^{15}$ Collectively, dietary sources are quite diverse and range from nuts, seeds, wholegrains, and legumes to fruits, herbs, and vegetables, and also include oils, tea, wine, and beer.

In foods, the flavonols, flavones, flavanones, isoflavones and anthocyanins occur mostly as glycosides (i.e., attached to a sugar moiety) rather than as aglycones (i.e., as free form compounds). ${ }^{16,17}$ Flavan-3-ol monomers on the other hand, are generally not present as glycosides; instead they occur most often in free form or as gallic acid esters. ${ }^{16,17}$ As the glycosidic flavonoids may be linked with many different sugars, ${ }^{17,18}$ to simplify analyses, these flavonoids are often quantified and reported as aglycone equivalents (abbreviated herein as AE). This is achieved by hydrolysing the flavonoid from their attached sugars and measuring the total content of the flavonoid aglycone present in a single food. ${ }^{17-19}$ Gallic acid esters of flavan-3-ols however, are often reported as such, without any conversion to aglycones; in turn, these values are often incorporated in total flavonoid intake calculations, while other subclasses are included as AE. ${ }^{19,20}$ When quantified exclusively as AE, it becomes apparent that only several dozen flavonoids are relatively common among foods and dietary intake. ${ }^{13,21}$ For example, in the French observational study SUpplémentation on VItamines et Minéraux AntioXydants (SU.VI.MAX), across the 4,942 participants, $>180$ different flavonoids were consumed by the cohort as part of their normal dietary intake, of which, $80 \%$ occurred as glycosides; the remainder occurring as aglycones or esters. ${ }^{21}$ Yet, when quantified entirely as AE, only 47 aglycones were commonly consumed. ${ }^{21}$ 
While the flavonoid subclass and compound make-up of plant foods differs within and between their genus, across species (e.g., apple varieties), the flavonoid subclasses present are more or less consistent. ${ }^{13}$ However, the absolute concentration of individual flavonoids in foods is strongly influenced by their growing, storage, and processing conditions. ${ }^{13}$ The flavonols are the most widely occurring of the flavonoids, being found in almost all fruits and vegetables. ${ }^{17}$ The richest flavonol sources include onions ( $>20 \mathrm{mg} / 100 \mathrm{~g} \mathrm{AE})$ and spinach $(>10 \mathrm{mg} / 100 \mathrm{~g}$ $\mathrm{AE})$; although, black tea ( $>3 \mathrm{mg} / 100 \mathrm{ml} \mathrm{AE})$ and apples $(>2 \mathrm{mg} / 100 \mathrm{~g} \mathrm{AE}$ with skin), are usually the more important dietary sources, as they are generally consumed in greater quantities. ${ }^{17,20}$ Anthocyanins are uniquely concentrated in berries (e.g., blueberries typically contain $>100 \mathrm{mg} / 100 \mathrm{~g} \mathrm{AE}$ ) however, are present in a several other foods (e.g., plum $>40$ to $>500 \mathrm{mg} / 100 \mathrm{~g}$ AE depending on species; kidney beans cooked $>3 \mathrm{mg} / 100 \mathrm{~g} \mathrm{AE}) .{ }^{17,20} \mathrm{On}$ the other hand, flavones are found in very limited quantities throughout foods. ${ }^{17}$ Examples of flavone food sources include whole grain wheat flour (e.g., sorghum flour $>2 \mathrm{mg} / 100 \mathrm{~g} \mathrm{AE}$ ), orange juice $(>5 \mathrm{mg} / 100 \mathrm{ml} \mathrm{AE})$ and extra virgin olive oil $(>1 \mathrm{mg} / 100 \mathrm{ml} \mathrm{AE}) .^{20}$ Flavanones are mostly concentrated in citrus fruits (e.g., orange $>40 \mathrm{mg} / 100 \mathrm{~g} \mathrm{AE}$ ) and their juices. ${ }^{19}$ Isoflavones are found in trace quantities throughout vegetables and grains; their only rich sources include soy bean products (e.g., edamame $>40 \mathrm{mg} / 100 \mathrm{~g} \mathrm{AE}) .{ }^{19,23}$ Lastly is the flavan3-ol subclass, which consist of several sub-groups including monomers (also termed catechins), as well as oligomers and polymers (also termed proanthocyanidins). Flavan-3-ols are also found in black tea in various oxidized oligomeric and polymeric forms, such as theaflavins and thearubigins, formed during tea leaf fermentation. ${ }^{24-26}$ Catechins and proanthocyanidins are present in high concentrations in dark chocolate $(>200 \mathrm{mg} / 100 \mathrm{~g})$, apples $(>100 \mathrm{mg} / 100 \mathrm{~g})$, red wine $(>50 \mathrm{mg} / 100 \mathrm{ml})$, nuts (e.g., almonds $>150 \mathrm{mg} / 100 \mathrm{~g})$ and berries (e.g., blueberry $>300 \mathrm{mg} / 100 \mathrm{~g}$ ) while, black tea is uniquely high in monomers, theaflavins and thearubigins $(>100 \mathrm{mg} / 100 \mathrm{ml}) \cdot{ }^{17,20,27}$ However, the measurement of thearubigins is often inaccurate,${ }^{21}$ and thus, they are often not included in intake estimates 
despite contributing most tea flavan-3-ols. ${ }^{20}$ As an aside, coffee is not a rich source of flavonoids ( $<0.5 \mathrm{mg} / 100 \mathrm{ml} \mathrm{AE}$ total flavonoids), however, it is abundant in phenolic acids. ${ }^{17}$ Ultimately, an individual's flavonoid exposure is dependent on their routine diet.

Globally, the average intake of total flavonoids is estimated at $\sim 400 \mathrm{mg} /$ day (calculated principally as $\mathrm{AE}$ though, not including thearubigins). ${ }^{28}$ However, dietary patterns and regional food consumption differs greatly between countries and continents so there can be large variability in the daily intake of flavonoids across the globe. ${ }^{28}$ While limitations exist in diet data collection methods and the estimation of flavonoid intake, ${ }^{13}$ some general estimates can be made. Yet, to preface these estimates, researchers tend to broadly estimate total flavonoid intake in terms of $\mathrm{AE}$, however, this includes free form flavan-3-ols and sometimes esterified flavan-3-ols as well. In the United States of America, the mean intake of total flavonoids varies from $\sim 250$ to $\sim 400 \mathrm{mg} /$ day $\mathrm{AE}$ (including thearubigins) while, in the United Kingdom, the average intake is reported to be $>1000 \mathrm{mg} /$ day $\mathrm{AE}$ (including thearubigins), which is more similar to intakes reported in Australia AE ( $\sim 650$ to $\sim 700 \mathrm{mg}$ /day including thearubigins). ${ }^{13,28}$ The differences likely reflect a high intake of black tea in the latter two countries where black tea may provide 60 to $80 \%$ of total flavonoid intake. ${ }^{13,28}$ Across mainland Europe, in Mediterranean countries (defined here as Greece, Spain, Italy, and Southern France), the mean intake of total flavonoids is $\sim 250$ to $\sim 400 \mathrm{mg}$ /day AE (not including thearubigins); the major contributor being fruit. ${ }^{28}$ In Nordic countries, such as Finland, average total flavonoid intake is $\sim 200$ to $\sim 250 \mathrm{mg} /$ day AE (not including thearubigins) while, to the Far East in China, $\sim 225$ $\mathrm{mg} /$ day AE (not including thearubigins) is generally consumed; the major contributor being tea, ${ }^{28}$ of which, green tea is a popular variety. ${ }^{29}$

As total daily flavonoid intake varies across the globe, so too does the consumption of flavonoid subclasses and their respective individual compound make-up. That being, in general, the flavan-3-ols are the most commonly consumed subclass, typically contributing 
upwards of $\sim 80 \%$ towards total flavonoid intake, followed by flavanones $(\sim 8-10 \%)$, anthocyanins ( 7-10\%), flavonols ( 7-9\%), flavones $(\sim 1-2 \%)$, and isoflavones which, at the population level, are generally (except in Asia) consumed in trace quantities. ${ }^{28}$ Yet, even within populations, differences in flavonoid intake occurs among subgroups. For example, in the European Prospective Investigation into Cancer, across Europe, higher flavonoid intake was associated with increasing age, education and physical activity as well as being female and a health-food consumer. ${ }^{30}$ Thus, across geographic regions and socio-demographic characteristics, differences in both the quantity and proportion of flavonoid-rich foods consumed results in a wide variability of flavonoid exposure among populations. ${ }^{28}$

\section{Calculation of flavonoid intake and how this has changed over the years}

Flavonoid intake in nutritional epidemiology has historically been estimated from food frequency questionnaires (FFQ) and diet histories using food composition tables. By the mid1970 's, emerging composition data, detailing a small range of foods, were used to calculate the first estimate of daily flavonoid intake from dietary origin. ${ }^{31,32}$ However, advances in analytical methods by the early 1990's enabled a more accurate measure of the flavonoid content of foods and up-dated composition tables were published by Hertog et al. in 1992 and 1993.18,33,34 These tables provide information on AEs of two flavones (i.e., luteolin and apigenin) and three flavonols (i.e., quercetin, myricetin and kaempferol) across $\sim 60$ foods and beverages. ${ }^{18,33,34}$

The food composition tables by Hertog et al. were used to estimate flavonoid intake in the Zutphen Elderly cohort in order to examine the association between flavonoid intake and CVD risk - the first prospective cohort study with this aim. ${ }^{5}$ This prompted an interest in assessing associations between habitual flavonoid consumption and CVD health outcomes and the data on flavonoid intakes developed by Hertog et al. were used as the cornerstone to estimate flavonoid intake in several prospective studies on CVD throughout the later 90's and early 2000 's. ${ }^{35-42}$ Interest in flavonoids other than flavones or flavonols was building, yet, at the turn 
of the century (1999 to 2000), accurate data on the flavan-3-ol content of foods was largely unavailable. ${ }^{43}$ At this time, Arts et al. measured the content of six flavan-3-ol monomers found in $>60$ foods and beverages. ${ }^{43,44}$ This spurred the investigation of flavan-3-ol intake and CVD risk in several prospective cohorts. ${ }^{45,46}$ However, a database detailing the gamut of flavonoids was lacking and studies were starting to integrate several smaller reports on the flavonoid composition of foods into their analyses. ${ }^{47}$

In 2003 the United States Department of Agriculture (USDA) released their database on the flavonoid content of 225 foods and beverages covering 26 of the most commonly occurring flavonoids (expressed as $\mathrm{AE}$, except for flavan-3-ols which were reported as free form aglycones and esters without conversion) throughout five subclasses (i.e., flavonols, flavones, flavanones, anthocyanins and flavan-3-ols (including catechins, theaflavins and thearubigins)). ${ }^{16}$ Around the same time, they also released their database on the isoflavone content of 128 foods covering three aglycones (i.e., daidzein, genistein, and glycitein) and in 2004 they released their database on the proanthocyanidin content of 205 foods. ${ }^{27,48}$ Following their initial release, the USDA databases have undergone several major updates. In 2007, the flavonoids database was updated to include 392 foods (Release 2.0), and in 2011, again expanded to include 500 foods (Release 3.0), although no other monomeric compounds were incorporated..$^{20}$ In 2008, the isoflavone database was updated to contain 557 foods (Release $2.0),{ }^{23}$ and in 2015 the proanthocyanidin database (Release 2.0) was updated to contain 283 foods; again neither incorporating further compounds. ${ }^{28}$ In 2014 the USDA released their extended database, which covers $\sim 2900$ foods and six major flavonoid subclasses except proanthocyandins ${ }^{49}$ Since their initial release, the USDA databases have been used regularly for estimating flavonoid intake in prospective cohort studies. ${ }^{50-57}$ However, different flavonoid glycosides are known to vary in their biological activity and a database detailing the range of flavonoids naturally occurring in foods was thus developed. 
The Phenol-Explorer database was released online in 2009. ${ }^{19}$ This database differs to the USDA databases in that it contains information on individual flavonoids as they are found in food (i.e., mainly as glycosides). ${ }^{19}$ Phenol-Explorer also contains information on other (poly)phenol classes, such as stilbenes, lignans and phenolic acids in addition to several other subclasses (e.g., curcuminoids), collectively providing information on 502 (poly)phenols in 452 foods. ${ }^{19}$ With regards to flavonoids, Phenol-Explorer compiles data that was (largely) measured utilising chromatography without hydrolysis (in which each individual flavonoid form, as found in food, is quantified) while, the USDA databases (are generally) compiled from analysis utilising chromatography after hydrolysis (which removes the glycosides enabling total quantification of the aglycones). ${ }^{16,19}$ To obtain AE using Phenol-Explorer, the user converts the glycoside or ester via molecular weight conversion into total aglycones. ${ }^{21}$ Alternatively, Phenol-Explorer also contains content values as measured by chromatography after hydrolysis, ${ }^{19}$ but, these data have been seldom used in cohort studies.

The first release of Phenol-Explorer contained information on $>270$ unique flavonoids. ${ }^{19}$ Importantly, unlike the USDA database, Phenol-Explorer does not include data on thearubigins however, both contain information on theaflavins. Phenol-Explorer was updated in 2012 (version 2.0) and again in 2013 (version 3.0) to contain information on flavonoid pharmacokinetics as well as the effect of food processing on flavonoid retention throughout food preparation. ${ }^{58,59}$ Like the USDA databases, Phenol-Explorer has been widely used to estimate flavonoid intake in epidemiological studies. ${ }^{60-64}$ Of note, Knaze et al. used PhenolExplorer to calculate the flavonoid content of foods and meals consumed in the European Prospective Investigation into Cancer; ${ }^{65}$ using retention factors, they generated the (poly)phenol composition of 19,899 raw and prepared, generic and multi-ingredient food items - the database of which is accessible online. ${ }^{65}$ While other databases detailing the flavonoid content of foods exist (e.g., EuroFIR-BASIS),${ }^{66}$ these have been much less frequently incorporated in epidemiological research. ${ }^{67}$ 
While there has been substantial advances in the completeness of flavonoid databases, such food composition tables are known to be limited by their coverage of food items and nutrients within foods..$^{13}$ In addition, the flavonoid composition of foods is variable as they are dependent on their variety, growing, storage, and processing or cooking conditions; thus such data are generally regarded as approximations of the contents of food. ${ }^{13,68}$ To this end, limited attention has been paid to the potential differences between flavonoid databases and the impact this may have on flavonoid intake estimates. In a recent method-comparison study, Ivey et al. studied the congruence between Phenol-Explorer (version 2.1) and both the USDA database for estimating flavonoid (Release 3.1) and proanthocyanidin (Release 1.0) consumption. ${ }^{69}$ From FFQs, flavonoid intake was estimated in AE, using all databases. ${ }^{69}$ The USDA and PhenolExplorer databases were highly correlated for total flavonoid intake and all subclasses $(r=$ $>0.8, P<0.001)$ except flavones $(r=0.34, P<0.001) .{ }^{69}$ However, the absolute mean intake values were significantly different between databases across all subclasses and total flavonoids. ${ }^{69}$ Despite these differences, when participants were classified as low, moderate and high consumers as estimated using both databases, in general, participants fell into the same intake categories. ${ }^{69}$ Thus, in epidemiology research, these issues need to be considered in systematic reviews and meta-analyses of data.

Regardless of the database used to calculate flavonoid intake, estimates can only be as accurate as the assessment tools used to quantify habitual diet. The quantification of habitual intake is complex and difficult because individuals eat a wide variety of different foods, in different combinations, in different amounts, in different frequencies. ${ }^{70}$ Until recent years, only a few main methods of assessing dietary intake have been available: the food diary, diet history, 24$\mathrm{hr}$ recall and FFQ. ${ }^{70,71}$ Of these, the FFQ is the most efficient and therefore most widely used. ${ }^{13,70}$ However, most FFQs used in prospective cohort investigations to date were constructed prior to the development of the USDA flavonoid databases and were not specifically designed for the measurement of flavonoid intake. ${ }^{13}$ As such, they may not 
optimally reflect the variability in flavonoid intake, due to the omission of some flavonoidbearing foods or the group of similar food items within the same question (e.g., berries), making it impossible to discern which specific food type/s the individual eats. ${ }^{13}$ Performance and validation studies of FFQs, specifically in regards to flavonoid intake, have seldom been conducted; while an FFQ may have been validated for other nutrients or food groups, this does not necessarily imply that it can reasonably assess intake of flavonoids. In this context, the extrapolation of absolute values from FFQs as accurate measures of intake is not usually justified. However, they may still provide a rank of usual consumption, allowing researchers to roughly separate out irregular (lower) vs regular (higher) consumers, in large samples, given the FFQ contains ample questions on major flavonoid bearing items. Ultimately, inaccurate dietary assessment biases results towards the null as noise in the data increases; the implications are that true associations are likely stronger than those reported. ${ }^{72}$ In more recent years, validation studies of FFQs for flavonoid intake have begun to emerge, ${ }^{73,74}$ however, it is hoped that nutrition science will look to shift to emerging technologies, such as biomarkers or smartphone methods, which may allow for a more robust assessment of dietary intake in the future. ${ }^{75}$

As described above, with advances in both knowledge and technology, methods used to estimate flavonoid intake in cohorts with data on habitual diet have changed over the last $\sim 25$ years (Figure 1). Prior to 2003 and the development of the USDA database and later, PhenolExplorer, total flavonoid intake reported in cohort studies ( $\sim 3$ to $25 \mathrm{mg} /$ day $)$ was calculated from the estimated intake of 5 to 10 compounds spanning 2 to 4 subclasses (flavonols, flavones, flavan-3-ols monomers and flavanones) $)^{5,36,46,47,76}$ This underestimation of flavonoid intake (and conceivable misclassification of participants) is a critical limitation of these early studies often not recognized in the literature. More recent studies present results for 5 to 7 flavonoid subclasses and have an average flavonoid intake ranging from approximately 130 to 700 $\mathrm{mg}$ /day. ${ }^{50,54,55,67,77,78}$ Further, flavonoid intake values between studies may not be directly comparable as the studies used different dietary assessment tools that were not necessarily 
validated for measuring flavonoids. These differences in calculating flavonoid intake must be

kept in mind; comparing findings from studies prior to development of the USDA and PhenolExplorer databases, to findings from studies post their release, is inherently difficult. In this review, we refer to a study as estimating total flavonoid intake, only if the estimate is a composite of $\geq 5$ subclasses; often, studies do not include isoflavones in their composite scores due to their low dietary occurrence in most populations. Where a study has estimated the sum of $\leq 4$ subclasses, we describe the sum of subclasses (e.g., intake of flavones plus flavonols etc.).

\section{[Insert Figure 1]}

Figure 1. Timeline of development of landmark flavonoid-food composition databases and the cumulative number of prospective cohort publications reporting on the intake of flavonoid subclasses and/or total consumption and cardiovascular disease risk. USDA, United States Department of Agriculture.

\section{Biological mechanisms of flavonoids in cardiovascular disease}

Following their digestion and absorption, flavonoid metabolites enter the blood stream where they may interact with molecular mechanisms mediating CVD. ${ }^{14,15,79}$ Over 170 biological effects have been reported for flavonoids and flavonoid-rich foods, reviewed recently by Williamson et al. ${ }^{14}$ Regarding their CVD-protective actions, the most recognized activity of flavonoids is their positive effects on the vasculature. In randomised controlled trials (RCTs), flavonoids and flavonoid-rich foods have been shown to lower blood pressure, ${ }^{80}$ improve endothelial function, ${ }^{81}$ and augment arterial stiffness. ${ }^{82}$ Most evidence from RCTs is for the flavan-3-ol subclass, which, in most populations, is the greatest contributor to total flavonoid intake. ${ }^{51,83,84}$

Meta-analyses of human RCTs have shown beneficial effects of flavonoids on major CVD risk factors. Blood pressure is one well-established risk factor for CVD. ${ }^{85}$ A meta-analysis of 91 
RCTs comparing the effect of a flavan-3-ol intervention with a control on blood pressure showed a significant decrease in SBP $(-1.46 \mathrm{~mm} \mathrm{Hg}, 95 \% \mathrm{CI}:-2.27,-0.65 \mathrm{mmHg})$ and DBP $(-0.99 \mathrm{~mm} \mathrm{Hg}, 95 \% \mathrm{CI}:-1.50,-0.45 \mathrm{~mm} \mathrm{Hg}) .{ }^{86}$ Another independent risk factor of CVD, endothelial dysfunction (impaired nitric oxide-mediated vasodilation), predicts atherothrombotic clinical events. ${ }^{87}$ Aside from impaired vasodilation, a dysfunctional endothelium may be permeable to atherogenic lipoproteins and secrete vascular adhesion molecules; critical initial steps in the development of an atherosclerotic lesion. ${ }^{88}$ A metaanalysis comparing the effects of flavan-3-ols with controls showed a significant increase in acute FMD response measured from 1 to $6 \mathrm{~h}(1.70 \%, 95 \% \mathrm{CI}: 1.31 \%, 2.08 \% ; 24 \mathrm{RCTs})$ and a significant improvement in chronic FMD measured after a few weeks of intervention $(1.21 \%$, 95\% CI: $0.70 \%, 1.73 \%$; 23 RCTs). ${ }^{86}$ Additionally, several RCTs have reported a decrease in arterial stiffness after a flavonoid-based intervention, independent of blood pressure changes. ${ }^{82}$ The exact mechanisms behind these improvements are yet to be established but may include enhanced nitric oxide bioavailability, ${ }^{89}$ increased expression of heme oxygenase- $1,{ }^{90}$ and inhibition of angiotensin-converting enzyme activity. ${ }^{91}$

Quantifying the effect of flavonoids on atherosclerosis itself in RCTs is inherently difficult due to slow disease initiation and progression. However, flavonoid interventions have been reported to lower LDL-cholesterol and triglycerides, and increase HDL-cholesterol in human studies, ${ }^{86}$ while animal and in vitro studies provide evidence that flavonoids improve inflammatory-status via the interference of pro-oxidant enzyme-signalling cascades or adhesion molecule expression. ${ }^{92}$ Unfortunately, fluctuations in background cytokine production and difficulty in detecting subtle changes in inflammatory status make this very difficult to assess in human intervention studies; a meta-analysis of RCTs on inflammatory biomarkers found no difference between flavan-3-ols and controls. ${ }^{86}$ Thus, in the absence of long-term clinical trials allowing for the long preclinical phase or quantifiable progression of 
atherosclerosis, observational prospective cohort studies provide us with the strongest evidence for the protective role of habitual flavonoid intakes in the manifestation of CVD.

\section{Flavonoid intake and cardiovascular disease risk: epidemiological evidence}

\section{a. Total cardiovascular disease}

Disease overview. Currently, the global burden of CVD accounts for approximately one third of all deaths and is the leading cause of mortality and disability worldwide. ${ }^{93,94}$ Total CVD is an umbrella term for a broad range of diseases of the heart and circulatory system, of which, the main underlying pathology is atherosclerosis. ${ }^{95,96}$ The major clinical manifestations of atherosclerotic CVD (ASCVD) include IHD, PAD, and ischemic stroke. ${ }^{97}$ Other CVDs, not aetiologically linked to atherosclerosis, include: structural heart disease, congenital heart disease, rheumatic heart disease, arrhythmias, and other disease of the arterial and venous system. ${ }^{98}$ In 2015, the Global Burden of Disease study estimated the worldwide prevalence of CVD for the most common causes of CVD death. ${ }^{94}$ They found there was $\sim 422.7$ million prevalent cases of CVD worldwide, of which, ASCVD was the main contributor with $\sim 154.7$ million cases of PAD $(\sim 36.6 \%), \sim 110.5$ million cases of $\operatorname{IHD}(\sim 26.2 \%)$ and $\sim 24.9$ million cases of ischemic stroke $(\sim 5.9 \%)$; the other $\sim 31.3 \%$ of prevalent cases constituted haemorrhagic and other strokes, hypertensive heart disease, cardiomyopathy, atrial fibrillation, endocarditis and other cardiovascular and circulatory diseases. ${ }^{94}$ In the same year, $\sim 17.9$ million people died from CVD, wherein, it is estimated that $\sim 66 \%$ died from ischemic stroke and IHD. ${ }^{94}$ In highincome countries, such as within the continent of North America, the proportion of the population dying of IHD and ischemic stroke, reaches as high as $\sim 75 \%$ of total CVD..${ }^{94}$ A range of in vitro and in vivo studies suggest that flavonoids exert beneficial effects on the arterial vascular system suggesting that CVDs of atherosclerotic origin may be particularly amenable to a flavonoid intervention..$^{79,99,100}$ While the bulk of existing literature on flavonoid intake and 
risk of total CVD includes CVDs of both atherosclerotic and non-atherosclerotic origins, as

415 highlighted above, cases are dominated by ASCVD.

Cohort characteristics. To date, at least 19 prospective cohorts (in 21 publications) have estimated flavonoid intake and the risk of total CVD incidence and/or mortality (Table 1). Nearly all studies reporting on CVD incidence defined this as the first non-fatal or fatal event. However, one study restricted outcomes to first time events resulting in hospitalisation, ${ }^{62}$ while another defined incidence as the first non-fatal event only. ${ }^{67}$ Around half of the studies defined total CVD as any type of CVD, mostly using the International Classification of Diseases (ICD) $9^{\text {th }}$ Revision, codes $390-459$, and their equivalent ICD-10 codes I00-I99 to identify case events ( $n=10$ publications). However, all such studies reported on morality outcomes - which is dominated by ASCVD (Table 1). At the same time, studies reporting on total CVD incidence, tend to restrict analysis to events of cerebrovascular disease and IHD, ${ }^{51,60,101-103}$ with some further incorporating death from any CVD (Table 1). ${ }^{43,66,89}$ Thus, they too are principally reporting on ASCVD. However, given this outcome definition, studies on CVD incidence tend to overlook non-fatal events of PAD; although PAD is the most prevalent ASCVD, a large proportion of PAD goes undiagnosed and therefore, in a cohort study, PAD usually represents proportionally less events. Other studies exclusively analysed events of ASCVD ${ }^{62,63}$ while two studies did not provide complete outcome definitions. ${ }^{67,78}$ At least one study presented analyses of total CVD as per several outcome definitions, of which we report the most relevant for this review. ${ }^{42}$ Effectively, while the definition of total CVD is somewhat heterogeneous, the cases are primarily, but not exclusively, ASCVD-related.

Other cohort characteristics were also heterogeneous. The cohorts vary in size (from 774 to 98,469 participants) as well as their follow-up timeframe (from 4.3 to 25 years) and location (United States of America $(n=5)$, Australia $(n=2)$, Asia $(n=2)$, and Europe $(n=10))$. Most cohorts recruited middle-aged participants (e.g., $\sim 50$ years), while some recruited older 
individuals (e.g., 65+ years). Habitual diet has been mainly assessed using FFQs ( $n=14$ cohorts) and flavonoid intake mostly estimated using the USDA ( $n=9$ publications) and/or Phenol-Explorer ( $n=6$ publications) databases (Table 1 ). Only one study indicated flavonoid intake was estimated as glycosides (and other naturally occurring structures); 60 all others appear to report intake as $\mathrm{AE}$ (including free form and potentially esterified flavan-3-ols).

Total flavonoids. As previously mentioned, in this review, we discuss a study as assessing total flavonoid intake, only if this estimate was a composite of $\geq 5$ subclasses. The association between such total flavonoid intake and total CVD incidence has been studied in at least five cohorts, while at least nine cohorts report on total CVD mortality. In 2017, Grosso et al. metaanalysed five of these cohorts reporting on total CVD mortality $(n=137,629$, with 5,351 CVD deaths), and found a trend towards a lower risk of death with higher total flavonoid intake (multivariable adjusted risk estimate for highest vs lowest intake category: $0.83,95 \%$ CI: 0.68 , 1.03). ${ }^{105}$ While high heterogeneity was observed among these studies $\left(I^{2}=67 \%\right)$, a subsequent sensitivity analysis (by systematic exclusion of cohorts) reduced heterogeneity $(R=20 \%, P=$ 0.29) and highlighted a significant association between total flavonoid intake and CVD mortality ( $n=4$ cohorts, $n=136,566, n=5,273$ deaths; multivariable adjusted risk estimate for highest vs lowest intake category: $0.88,95 \%$ CI: $0.79,0.98) .{ }^{105}$ The excluded study by Ivey et al. reported the strongest association of all studies (multivariable adjusted risk estimate for highest vs lowest intake category: $0.32,95 \%$ CI: $0.16,0.61) .{ }^{77}$ Ivey et al. undertook analysis in the oldest cohort in the meta-analysis ( $n=1,063$, mean age: $80 \pm 3$ years at baseline) with $\sim 30 \%$ of the sample experiencing pre-existing CVD at baseline, which may explain why such a strong association was observed. That is, participants would have been at very high risk of dying of CVD (7.3\% died of CVD). ${ }^{77}$ The Grosso et al. meta-analysis found no evidence of publication bias, which was supported by a similar meta-analysis published in the same year. ${ }^{106}$ However, this finding may not be overly convincing as both meta-analyses included a relatively small number of cohorts. ${ }^{106}$ Grosso et al. also found an association, albeit non-significant, 
between CVD mortality and total flavonoid intake when the meta-analysis was confined exclusively to studies adjusting for dietary confounders in addition to sociodemographic factors $(n=3$ studies; multivariable adjusted risk estimate for highest vs lowest intake category: $0.71,95 \%$ CI: $\left.0.33,1.51 ; I^{2}=79 \%\right)$. Consequently, cofounding from unmeasured nutritional components may have biased results, although conclusions are limited due to a small sample size and wide confidence intervals. ${ }^{105}$ Since the Grosso et al. meta-analysis, a further three studies on total flavonoid intake and CVD mortality have been published with results trending towards a lower associated risk of CVD mortality with higher flavonoid intake. ${ }^{51,52,61}$ Unlike for total CVD mortality, a meta-analysis on total flavonoid intake and CVD incidence has not been published. However, significant inverse relationships emerge in three of the five cohorts reporting on this outcome. While other meta-analyses in the field have pooled heterogeneous flavonoid exposures and outcome measures, overall their findings suggest that flavonoid-rich dietary patterns are associated with a lower risk of CVD events. ${ }^{107,108}$

Flavonoid subclasses. The impact of individual flavonoid subclasses on total CVD risk has been studied across incidence ( $n=9$ cohorts) and mortality ( $n=14$ cohorts) outcomes. The results typically report an inverse association reaching statistical significance for all subclasses in at least one or more cohorts (Table 1). Grosso et al. meta-analysed several of the cohorts reporting on CVD mortality. ${ }^{105}$ A significantly lower risk of death was found for the highest compared to the lowest intake categories of anthocyanins $(n=5$ cohorts; $\mathrm{RR}=0.89,95 \% \mathrm{CI}$ : $0.83,0.95 ; I^{2}=0 \%$ ), catechins (termed flavan-3-ols in their manuscript; $n=7$ cohorts; $\mathrm{RR}=$ $\left.0.82,95 \% \mathrm{CI}: 0.71,0.95 ; I^{2}=53 \%\right)$, proanthocyanidins $(n=4$ cohorts; $\mathrm{RR}=0.89,95 \% \mathrm{CI}$ : $\left.0.81,0.97 ; I^{2}=0 \%\right)$, flavonols $\left(n=6\right.$ cohorts; $\mathrm{RR}=0.79,95 \%$ CI: $\left.0.63,0.99 ; I^{2}=67 \%\right)$, flavones $\left(n=5 ; \mathrm{RR}=0.85,95 \% \mathrm{CI}: 0.75,0.96 ; I^{2}=25 \%\right)$, and flavanones $(n=5$ cohorts; $\mathrm{RR}$ $=0.84,95 \%$ CI: $\left.0.73,0.96 ; I^{2}=34 \%\right)$ but not isoflavones $(n=6$ cohorts; $\mathrm{RR}=1.01,95 \% \mathrm{CI}$ : $0.94,1.08 ; R^{2}=0 \%$ ) in multivariable adjusted models. ${ }^{105}$ The high heterogeneity observed for flavonols and catechins was reportedly due to a study by Mursu et al., 55 which after removal, 
reduced heterogeneity without any material impact on the results. These findings were in agreement with two other recent meta-analyses on CVD mortality risk and intake of anthocyanins and flavan-3-ols. ${ }^{86,109}$ Since these meta-analyses, at least three further prospective cohorts have been published on each subclass (except isoflavones); results typically trending towards a beneficial association, though, reaching significance only for anthocyanin intake and lower CVD mortality risk in one cohort (Table 1). While less research has focused on CVD incidence, it is suspected that outcomes would echo CVD mortality findings.

Dose-response. The intake of flavonoids associated with significant benefit has been the subject of investigation. In a dose-response meta-analysis, Grosso et al. report a $4 \%$ lower risk of CVD mortality for every $\sim 100 \mathrm{mg} /$ day increment in total flavonoid intake ( $n=5$ cohorts, $n$ $=137,629$, total $\mathrm{CVD}$ deaths $=5,351$; multivariable adjusted risk estimate: $0.96,95 \%$ CI: 0.92 , $\left.1.00 ; P^{2}=68 \%\right) .{ }^{105}$ While a significant linear relationship emerged over the range of intakes studied $\left(P_{\text {linearity }}<0.001\right)$ it is unlikely that the true relationship maintains linearity indefinitely. It is more plausible that there is a plateau in the trend somewhere, where further consumption affords no added benefit, although the point of plateau likely differs in populations with different underlying risk. Nonetheless, it appears that only moderate intakes may be needed to positively affect health outcomes, and that higher intakes likely afford no added benefit. Results supporting this assertion can be seen in analyses from the Cancer Prevention Study II Nutrition Cohort $(n=98,469),{ }^{54}$ and the Danish Diet Cancer and Health Cohort $(n=56,048),{ }^{61}$ both of which show nonlinear dose-response thresholds, somewhere around $\sim 250$ to $\sim 500 \mathrm{mg} / \mathrm{day} \mathrm{AE}$ of total flavonoid intake. However, extrapolation of absolute values from FFQs is not usually possible unless a study has validation data. ${ }^{110}$

Effect modification. As indicated, the beneficial associations of dietary patterns high in flavonoids might differ, in different population subgroups. It has previously been hypothesized that the association between flavonoid intake and CVD mortality may be stronger in persons 
516 at a higher risk of CVD. Early findings of a study by Mink et al. weakly supported this 517 hypothesis. ${ }^{50}$ In their analysis, significant inverse associations between flavanone intake (but 518 not other subclasses or total intake) and CVD mortality were found in ever-smokers, but not never-smokers following multivariable adjustment for age and energy. ${ }^{50}$ Bondonno et al., then showed a lower risk of all-cause mortality (31\% were CVD-related) for higher flavonoid intakes only amongst participants with at least one early mortality risk factor (smoking, high alcohol consumption, no regular exercise or obesity). ${ }^{51}$ Following on from this, more results by Bondonno et al. show clear effect modification by smoking status and alcohol intake at baseline although, not for sex, physical activity, or diabetes following multivariable adjustment for lifestyle factors. ${ }^{61}$ That is, the inverse association between total flavonoid intake and total CVD mortality appeared to differ in smokers in contrast to non-smokers as well as in high alcohol over low alcohol consumers suggesting dietary flavonoid intake may partially mitigate harmful effects of such activities (although, the smoking and high alcohol intake groups would remain at higher overall risk of CVD compared to their counterparts). Given this finding, it stands to reason that associations between flavonoid intake and CVD may appear to be stronger in cohorts that have a higher proportion of smokers and high alcohol consumers. It is also plausible that further unidentified effect modifiers exist, such as in racial or ethnic populations, which warrants further investigation. However, as "at risk" populations have a higher underlying risk of $\mathrm{CVD}$, these findings require investigation on an absolute, as well as, a relative scale. 
Table 1. Summary of prospective cohort studies investigating the association of flavonoid intake with total cardiovascular disease incidence and mortality

\begin{tabular}{|c|c|c|c|c|c|c|c|c|c|c|c|c|c|c|c|}
\hline $\begin{array}{l}\text { Author, } \\
\text { year }\end{array}$ & $\begin{array}{l}\text { Country } \\
\text { (cohort) }\end{array}$ & $\begin{array}{c}n \\
\text { (population) }\end{array}$ & $\begin{array}{c}\text { Base- } \\
\text { line age } \\
\text { (yrs) }\end{array}$ & $\begin{array}{c}\text { Follow- } \\
\text { up } \\
\text { (yrs) }\end{array}$ & $\begin{array}{l}\text { Outcome } \\
\text { assessment }\end{array}$ & $\begin{array}{l}\text { Flavonoid } \\
\text { intake } \\
\text { estimate }^{a}\end{array}$ & Analysis & Exposure $^{b}$ & $\begin{array}{c}\text { Intake } \\
\text { (low vs high) } \\
\text { mg/day }\end{array}$ & \multicolumn{3}{|c|}{ Total CVD incidence $^{c}$} & \multicolumn{3}{|c|}{$\begin{array}{l}\text { Total CVD mortality } \\
\end{array}$} \\
\hline Bondonno & Denmark & $\begin{array}{l}56,048 \\
\end{array}$ & 56 & 23 & ICD-10 & FFQ/ & $\begin{array}{l}\text { Hazard } \\
\end{array}$ & Anthocyanidin: & $<9.6 \mathrm{vs} \geq 53.2$ & - & - & - & 3,981 & $0.90(0.82,0.99)$ & - \\
\hline \multirow[t]{5}{*}{$2019^{61}$} & (Danish Diet, & $(M \& F)$ & & & I00-199 & PE & ratio by & Catechin: & $<20.5$ vs $\geq 281.4$ & & - & - & & $0.97(0.89,1.06)$ & - \\
\hline & Health study) & & & & & & & Flavanone: & $<6.0$ vs $\geq 49.0$ & & - & - & & $0.96(0.88,1.05)$ & - \\
\hline & & & & & & & & Flavone: & $<3.0$ vs $\geq 8.8$ & & - & - & & $0.97(0.88,1.06)$ & - \\
\hline & & & & & & & & Flavonol: & $<20.5 \mathrm{vs} \geq 82.5$ & & - & - & & $0.95(0.87,1.05)$ & - \\
\hline & & & & & & & & Total: & $<250.8$ vs $\geq 908.5$ & & - & - & & $0.97(0.88,1.06)$ & - \\
\hline \multirow{6}{*}{$\begin{array}{l}\text { Bondonno } \\
2019^{51}\end{array}$} & Australia & 2,349 & 65 & 14 & ICD-9 & $\mathrm{FFQ} /$ & Hazard & Anthocyanidin: & $<3.8 \mathrm{vs} \geq 9.2$ & - & - & - & 213 & $0.80(0.53,1.18)$ & - \\
\hline & & & & & & & & Flavanone: & $<10.3$ vs $\geq 38.9$ & & - & - & & $0.87(0.61,1.25)$ & - \\
\hline & & & & & & & & Flavone: & $<0.72$ vs $\geq 1.32$ & & - & - & & $0.80(0.56,1.15)$ & - \\
\hline & & & & & & & & Flavonol: & $<25.0 \mathrm{vs} \geq 42.3$ & & - & - & & $1.01(0.71,1.44)$ & - \\
\hline & & & & & & & & Isoflavone: & $<0.81 \mathrm{vs} \geq 1.23$ & & - & - & & $1.16(0.81,1.66)$ & - \\
\hline & & & & & & & & Total: & $<599.9$ vs $\geq 1106$ & & - & - & & $0.98(0.68,1.41)$ & - \\
\hline \multirow{5}{*}{$\begin{array}{l}\text { Dalgaard } \\
2019^{62}\end{array}$} & Denmark & 53,552 & 56 & 23 & Hospital & $\mathrm{FFQ} /$ & Hazard & Anthocyanidin: & $<9$ vs $\geq 53$ & 8,773 & $1.02(0.95,1.09)$ & - & - & - & - \\
\hline & (Danish Diet, & $(M \& F)$ & & & admission for & $\mathrm{PE}$ & ratio by & Catechin: & $<21$ vs $\geq 282$ & & $0.91(0.86,0.97)$ & - & & - & - \\
\hline & Cancer \& & & & & atherosclerotic & & quintiles & PA + theaflavin: & $<137$ vs $\geq 435$ & & $0.87(0.81,0.93)$ & - & & - & - \\
\hline & Health study) & & & & CVD; ICD-10 I20- & & & Flavanone: & $<6$ vs $\geq 50$ & & $0.97(0.90,1.04)$ & - & & - & - \\
\hline & & & & & & & & Total: & $<253$ vs $\geq 912$ & & $0.90(0.85,0.97)$ & - & & - & - \\
\hline $\begin{array}{l}\text { Mendonca } \\
2019^{104}\end{array}$ & $\begin{array}{c}\text { Spain } \\
\text { (SUN cohort) }\end{array}$ & $\begin{array}{c}17,065 \\
(\mathrm{M} \& \mathrm{~F})\end{array}$ & 37 & 10 & $\begin{array}{l}\text { MI, stroke or } \\
\text { death from CVD } \\
\text { causes }\end{array}$ & $\begin{array}{c}\mathrm{FFQ} / \\
\mathrm{PE}\end{array}$ & $\begin{array}{l}\text { Hazard } \\
\text { ratio by } \\
\text { quintile }\end{array}$ & Total: & $\overline{\mathrm{x}}=186$ vs $\overline{\mathrm{x}}=772$ & 113 & $0.53(0.29,0.98)$ & 0.09 & - & - & - \\
\hline & France & 84,158 & 44 & 5 & Stroke, MI, & $3 \times 24 \mathrm{hr}$ & Hazard & Anthocyanin: & $\overline{\mathrm{x}}=27.5$ vs $\overline{\mathrm{x}}=55.0$ & 602 & $0.67(0.53,0.85)$ & 0.0007 & - & - & - \\
\hline \multirow[t]{7}{*}{$2018^{60}$} & (Nutrinet- & $(\mathrm{M} \& \mathrm{~F})$ & & & acute coronary & diet record/ & ratio by & Catechin: & $\overline{\mathrm{x}}=75.4$ vs $\overline{\mathrm{x}}=178$ & & $0.76(0.62,0.94)$ & 0.01 & & - & - \\
\hline & & & & & syndrome, or & $\mathrm{PE}^{* * * *}$ & tertiles & Theaflavin: & $\overline{\mathrm{x}}=10.3$ vs $\overline{\mathrm{x}}=24.5$ & & $0.89(0.67,1.18)$ & 0.41 & & - & - \\
\hline & & & & & angioplasty & & & PA: & $\overline{\mathrm{x}}=33.7$ vs $\bar{x}=71.7$ & & $0.85(0.68,1.01)$ & 0.05 & & - & - \\
\hline & & & & & & & & Flavanone: & $\overline{\mathrm{x}}=30.1$ vs $\overline{\mathrm{x}}=30.7$ & & $0.99(0.81,1.24)$ & 0.93 & & - & - \\
\hline & & & & & & & & Flavone: & $\overline{\mathrm{x}}=23.3$ vs $\overline{\mathrm{x}}=27.6$ & & $1.12(0.90,1.40)$ & 0.32 & & - & - \\
\hline & & & & & & & & Flavonol: & $\overline{\mathrm{x}}=46.5$ vs $\overline{\mathrm{x}}=86.3$ & & $0.77(0.61,0.98)$ & 0.02 & & - & - \\
\hline & & & & & & & & Isoflavone: & $\overline{\mathrm{x}}=4.5$ vs $\overline{\mathrm{x}}=11.5$ & & $0.98(0.80,1.20)$ & 0.89 & & - & - \\
\hline & USA & 93,145 & 36 & 18 & ICD-8 & $\mathrm{FFQ} /$ & Hazard & Anthocyanidin: & $<3 \mathrm{vs} \geq 17$ & - & - & - & 189 & $0.85(0.50,1.43)$ & - \\
\hline \multirow[t]{6}{*}{$2017^{52}$} & (NHS II) & (F) & & & $390-458$ \& & USDA & ratio by & Catechin: & $<12 \mathrm{vs} \geq 86$ & & - & - & & $0.75(0.49,1.16)$ & - \\
\hline & & & & & ICD-9 & & quintiles & PA: & $<79$ vs $\geq 356$ & & - & - & & $0.77(0.49,1.20)$ & - \\
\hline & & & & & $390-459$ & & & Flavanone: & $<9$ vs $\geq 51$ & & - & - & & $1.10(0.69,1.76)$ & - \\
\hline & & & & & & & & Flavone: & $<0.7 \mathrm{vs} \geq 2.2$ & & - & - & & $1.15(0.72,1.83)$ & - \\
\hline & & & & & & & & Flavonol: & $<9 \mathrm{vs} \geq 26$ & & - & - & & $0.91(0.58,1.41)$ & - \\
\hline & & & & & & & & Total: & $<138$ vs $\geq 518$ & & - & - & & $0.83(0.53,1.29)$ & - \\
\hline
\end{tabular}


Table 1. Continued: Summary of prospective cohort studies investigating the association of flavonoid intake with total CVD incidence and mortality

\begin{tabular}{|c|c|c|c|c|c|c|c|c|c|c|c|c|c|c|c|}
\hline \multirow[b]{2}{*}{$\begin{array}{l}\text { Author, } \\
\text { year }\end{array}$} & \multirow[b]{2}{*}{$\begin{array}{l}\text { Country } \\
\text { (cohort) }\end{array}$} & \multirow[b]{2}{*}{$\begin{array}{c}n \\
\text { (population) }\end{array}$} & \multirow[b]{2}{*}{$\begin{array}{c}\text { Base- } \\
\text { line age } \\
\text { (yrs) }\end{array}$} & \multirow[b]{2}{*}{$\begin{array}{c}\text { Follow- } \\
\text { up } \\
\text { (yrs) }\end{array}$} & \multirow[b]{2}{*}{$\begin{array}{l}\text { Outcome } \\
\text { assessment }\end{array}$} & \multirow[b]{2}{*}{$\begin{array}{c}\text { Flavonoid } \\
\text { intake } \\
\text { estimate }^{a}\end{array}$} & \multirow[b]{2}{*}{ Analysis } & \multirow[b]{2}{*}{ Exposure $^{b}$} & \multirow[b]{2}{*}{$\begin{array}{c}\text { Intake } \\
\text { (low vs high) } \\
\text { mg/day }\end{array}$} & \multicolumn{3}{|c|}{ Total CVD incidence $^{c}$} & \multicolumn{3}{|c|}{ Total CVD mortality } \\
\hline & & & & & & & & & & $n$ & $\begin{array}{l}\text { Fully adjusted } \\
\text { estimate }(95 \% \mathrm{Cl}) \\
{[\text { high vs low] }}\end{array}$ & $\begin{array}{l}P \text { for } \\
\text { trend }\end{array}$ & $n$ & $\begin{array}{c}\text { Fully adjusted } \\
\text { estimate }\left(95 \% \mathrm{Cl}^{d}\right) \\
{[\text { high vs low] }]^{d}}\end{array}$ & $\begin{array}{l}P \text { for } \\
\text { trend }\end{array}$ \\
\hline $\begin{array}{l}\text { Dower } \\
2016^{111}\end{array}$ & $\begin{array}{l}\text { Netherlands } \\
\text { (Zutphen) }\end{array}$ & $\begin{array}{l}774 \\
\text { (M) }\end{array}$ & $\begin{array}{c}65 \\
\text { to } 84\end{array}$ & 25 & $\begin{array}{c}\text { ICD-9 } \\
390-459 \\
\& 798.2\end{array}$ & $\begin{array}{c}\text { Diet } \\
\text { history/Arts } \\
\text { et all } 43,44\end{array}$ & $\begin{array}{l}\text { Hazard } \\
\text { ratio by } \\
\text { tertiles }\end{array}$ & Catechin & $<9.4 \mathrm{vs} \geq 19.5$ & - & - & - & 329 & $0.79(0.58,1.08)$ & - \\
\hline $\begin{array}{l}\text { Ivey } \\
2015^{77}\end{array}$ & $\begin{array}{c}\text { Australia } \\
\text { (CAIFOS) }\end{array}$ & $\begin{array}{c}1,063 \\
\text { (post- } \\
\text { menopause } \\
\text { women) }\end{array}$ & $>75$ & 5 & $\begin{array}{c}\text { ICD-9 } \\
390-459 \& \\
\text { ICD-10 } \\
\text { I00-I99 }\end{array}$ & $\begin{array}{l}\text { FFQ/ } \\
\text { PE and } \\
\text { USDA }\end{array}$ & $\begin{array}{l}\text { Hazard } \\
\text { ratio by } \\
\text { tertiles }\end{array}$ & $\begin{array}{r}\text { Total (USDA): } \\
\text { Total (PE): }\end{array}$ & $\begin{array}{l}<547 \text { vs } \geq 813 \\
<525 \text { vs } \geq 788\end{array}$ & - & - & - & 78 & $\begin{array}{l}0.34(0.17,0.69) \\
0.32(0.16,0.61)\end{array}$ & - \\
\hline $\begin{array}{l}\text { Jacques } \\
2015^{53}\end{array}$ & $\begin{array}{c}\text { USA } \\
\text { (Framingham } \\
\text { Offspring } \\
\text { Cohort) }\end{array}$ & $\begin{array}{c}2,880 \\
(\mathrm{M} \& \mathrm{~F})\end{array}$ & 54 & 14.9 & $\begin{array}{l}\text { Stroke, TIA, } \\
\text { CHD, heart } \\
\text { failure or } \\
\text { peripheral } \\
\text { vascular disease }\end{array}$ & $\begin{array}{l}\text { FFQ/ } \\
\text { USDA }\end{array}$ & $\begin{array}{l}\text { Relative risk } \\
\text { for each } \\
2.5 \text {-fold } \uparrow \text { in } \\
\text { daily intake }\end{array}$ & $\begin{array}{r}\text { Anthocyanidin: } \\
\text { Catechin: } \\
\text { PA + ODC: } \\
\text { Flavanone: } \\
\text { Flavone: } \\
\text { Flavonol: } \\
\text { Total: }\end{array}$ & $\begin{array}{l}- \\
- \\
- \\
- \\
-\end{array}$ & $\begin{array}{rl}518 & 0 \\
0 \\
0 \\
1 \\
1 \\
1 \\
0 \\
0\end{array}$ & $\begin{array}{l}0.95(0.86,1.05) \dagger \\
0.97(0.88,1.05) \dagger \\
0.93(0.84,1.02) \dagger \\
1.00(0.92,1.09) \dagger \\
1.00(0.82,1.21) \dagger \\
0.89(0.75,1.06) \dagger \\
0.93(0.82,1.06) \dagger\end{array}$ & $\begin{array}{l}0.30 \\
0.43 \\
0.13 \\
0.97 \\
0.99 \\
0.20 \\
0.26\end{array}$ & - & $\begin{array}{l}- \\
- \\
- \\
- \\
- \\
-\end{array}$ & $\begin{array}{l}- \\
- \\
- \\
- \\
- \\
-\end{array}$ \\
\hline $\begin{array}{l}\text { Ponzo } \\
2015^{67}\end{array}$ & $\begin{array}{c}\text { Italy } \\
\text { (no cohort } \\
\text { name) }\end{array}$ & $\begin{array}{c}1,658 \\
(\mathrm{M} \& \mathrm{~F})\end{array}$ & $\begin{array}{l}45 \\
\text { to } 64\end{array}$ & 12 & $\begin{array}{c}\text { Mortality: } \\
\text { ICD-9 } \\
390-459 \\
\& 798.1 ; \text { in } \\
\text { codes specified } \\
\text { for incidence }\end{array}$ & $\begin{array}{l}\text { FFQ/ } \\
\text { Mostly } \\
\text { USDA }\end{array}$ & $\begin{array}{l}\text { Hazard } \\
\text { ratio by } \\
\text { tertiles }\end{array}$ & $\begin{array}{r}\text { Anthocyanidin: } \\
\text { Catechin + ODC: } \\
\text { PA: } \\
\text { Flavanone: } \\
\text { Flavone: } \\
\text { Flavonol: } \\
\text { Isoflavone: } \\
\text { Total: }\end{array}$ & $\begin{array}{c}- \\
- \\
- \\
- \\
- \\
- \\
<191.5 \text { vs } \geq 322.3\end{array}$ & $\begin{array}{ll}125 \\
0 \\
c \\
c \\
c \\
c\end{array}$ & $\begin{array}{l}0.56(0.36,0.89)^{*} \\
0.42(0.26,0.6)^{*} \\
0.43(0.27,0.70)^{*} \\
0.48(0.29,0.77)^{*} \\
0.66(0.40,1.09)^{*} \\
0.53(0.34,0.83)^{*} \\
0.77(0.49,1.21)^{*} \\
0.46(0.28,0.75)^{*}\end{array}$ & $\begin{array}{l}- \\
- \\
- \\
- \\
- \\
- \\
-\end{array}$ & 84 & $\begin{array}{l}0.67(0.38,1.18) \\
0.72(0.41,1.26) \\
0.80(0.44,1.46) \\
0.66(0.37,1.17) \\
0.83(0.45,1.52) \\
0.72(0.41,1.27) \\
0.74(0.41,1.36) \\
0.83(0.46,1.51)\end{array}$ & $\begin{array}{l}- \\
- \\
- \\
- \\
- \\
- \\
-\end{array}$ \\
\hline $\begin{array}{l}\text { Vogiatz- } \\
\text { ogloua } \\
2015^{103}\end{array}$ & $\begin{array}{l}\text { UK } \\
\text { (EPIC) }\end{array}$ & $\begin{array}{l}\text { M: } 11,252 \\
\text { F: } 13,633\end{array}$ & $\begin{array}{l}40 \\
\text { to } 75\end{array}$ & 11.1 & $\begin{array}{c}\text { ICD-9 } \\
410-448 \text { \& } \\
\text { ICD-10 } \\
\text { I10-179 }\end{array}$ & $\begin{array}{l}\text { 7-day } \\
\text { diary/ } \\
\text { Flaviola } \\
\text { Flavanol } \\
\text { Database }\end{array}$ & $\begin{array}{l}\text { Hazard } \\
\text { ratio by } \\
\text { quintiles }\end{array}$ & $\begin{array}{l}\text { Males: } \\
\text { Total flavan-3-ol: } \\
\text { Females: } \\
\text { Total flavan-3-ol: }\end{array}$ & $\begin{array}{l}\bar{x}=198 \text { vs } \bar{x}=2008 \\
\bar{x}=179 \text { vs } \bar{x}=1828\end{array}$ & 4,060 & $0.90(0.81,0.99)$ & 0.176 & 1,154 & $0.95(0.74,1.20)$ & 0.607 \\
\hline $\begin{array}{l}\text { Talaei } \\
2014^{112}\end{array}$ & $\begin{array}{c}\text { Singapore } \\
\text { (Singapore } \\
\text { Chinese Health } \\
\text { Study) }\end{array}$ & $\begin{array}{c}63,257 \\
(\mathrm{M} \& \mathrm{~F})\end{array}$ & $\begin{array}{l}45 \\
\text { to } 74\end{array}$ & 14.7 & $\begin{array}{c}\text { ICD-9 } \\
390-459\end{array}$ & $\begin{array}{c}\mathrm{FFQ} / \\
\text { Singapore } \\
\text { Composition } \\
\text { Database }^{113}\end{array}$ & $\begin{array}{l}\text { Hazard } \\
\text { ratio by } \\
\text { quartile }\end{array}$ & Isoflavone: & $\overline{\mathrm{x}}=5.4$ vs $\overline{\mathrm{x}}=36.1$ & - & - & - & 4,780 & $1.00(0.91,1.10)$ & 0.83 \\
\hline $\begin{array}{l}\text { Tresserra- } \\
\text { Rimbau } \\
2014^{64}\end{array}$ & $\begin{array}{c}\text { Spain } \\
\text { (PREDIMED) }\end{array}$ & $\begin{array}{c}7,172 \\
(\mathrm{M} \& \mathrm{~F})\end{array}$ & $\sim 67$ & 4.3 & $\begin{array}{l}\text { MI, stroke } \\
\text { or death from } \\
\text { CVD causes }\end{array}$ & $\mathrm{FFQ/PE}$ & $\begin{array}{l}\text { Hazard } \\
\text { ratio by } \\
\text { quintiles }\end{array}$ & $\begin{array}{r}\text { Anthocyanin: } \\
\text { Total flavan-3-ol: } \\
\text { Flavanone: } \\
\text { Flavone: } \\
\text { Flavonol: } \\
\text { Total: }\end{array}$ & $\begin{array}{l}11.8 \text { vs } 74.6 \\
90 \text { vs } 263 \\
28 \text { vs } 247 \\
20 \text { vs } 67 \\
56 \text { vs } 124 \\
273 \text { vs } 1170\end{array}$ & 273 & $\begin{array}{l}0.67(0.39,1.13) \\
0.40(0.23,0.72) \\
1.09(0.68,1.74) \\
1.07(0.64,1.80) \\
0.69(0.40,1.19) \\
0.71(0.43,1.18)\end{array}$ & $\begin{array}{l}0.05 \\
0.003 \\
0.54 \\
0.72 \\
0.08 \\
0.16\end{array}$ & - & $\begin{array}{l}- \\
- \\
- \\
- \\
- \\
-\end{array}$ & $\begin{array}{l}- \\
- \\
- \\
- \\
- \\
-\end{array}$ \\
\hline
\end{tabular}


550 Table 1. Continued: Summary of prospective cohort studies investigating the association of flavonoid intake with total CVD incidence and mortality

\begin{tabular}{|c|c|c|c|c|c|c|c|c|c|c|c|c|c|c|c|}
\hline \multirow[b]{2}{*}{$\begin{array}{l}\text { Author, } \\
\text { year }\end{array}$} & \multirow[b]{2}{*}{$\begin{array}{l}\text { Country } \\
\text { (cohort) }\end{array}$} & \multirow[b]{2}{*}{$\begin{array}{c}n \\
\text { (population) }\end{array}$} & \multirow[b]{2}{*}{$\begin{array}{c}\text { Base- } \\
\text { line age } \\
\text { (yrs) }\end{array}$} & \multirow[b]{2}{*}{$\begin{array}{c}\text { Follow- } \\
\text { up } \\
\text { (yrs) }\end{array}$} & \multirow[b]{2}{*}{$\begin{array}{l}\text { Outcome } \\
\text { assessment }\end{array}$} & \multirow[b]{2}{*}{$\begin{array}{l}\text { Flavonoid } \\
\text { intake } \\
\text { estimate }^{a}\end{array}$} & \multirow[b]{2}{*}{ Analysis } & \multirow[b]{2}{*}{ Exposure $^{b}$} & \multirow[b]{2}{*}{$\begin{array}{c}\text { Intake } \\
\text { (low vs high) } \\
\text { mg/day }\end{array}$} & \multicolumn{3}{|c|}{ Total CVD incidence $^{c}$} & \multicolumn{3}{|c|}{ Total CVD mortality } \\
\hline & & & & & & & & & & $n$ & $\begin{array}{l}\text { Fully adjusted } \\
\text { estimate }(95 \% \mathrm{CI}) \\
{[\text { high vs low] }]^{d}}\end{array}$ & $\begin{array}{l}P \text { for } \\
\text { trend }\end{array}$ & $n$ & $\begin{array}{l}\text { Fully adjusted } \\
\text { estimate }(95 \% \mathrm{CI}) \\
\text { [high vs low }]^{d} \\
\end{array}$ & $\begin{array}{l}P \text { for } \\
\text { trend }\end{array}$ \\
\hline $\begin{array}{l}\text { Ivey } \\
\end{array}$ & Australia & 1,063 & $>75$ & 5 & ICD-9 & $\mathrm{FFQ} /$ & Odds ratio by & Anthocyanidin: & $<23$ vs $>41$ & - & - & - & 64 & $0.67(0.33,1.34)$ & 0.35 \\
\hline \multirow[t]{6}{*}{$2013^{63}$} & (CAIFOS) & (post- & & & 410-414, & USDA & tertile & Catechin + ODC: & $<296$ vs $>563$ & & - & - & & $0.34(0.17,0.70)$ & 0.01 \\
\hline & & menopaus & & & $428,433-$ & & & PA: & $<140$ vs $>229$ & & - & - & & $0.62(0.32,1.20)$ & 0.22 \\
\hline & & al women) & & & $438 \&$ & & & Flavanone: & $<32$ vs $>61$ & & - & - & & $0.55(0.28,1.09)$ & 0.16 \\
\hline & & & & & $440-444$ & & & Flavone: & $<2$ vs $>3$ & & - & - & & $0.56(0.29,1.12)$ & 0.20 \\
\hline & & & & & & & & Flavonol: & $<24$ vs $>35$ & & - & - & & $0.27(0.13,0.59)$ & 0.01 \\
\hline & & & & & & & & Isoflavone: & $<3$ vs $>4$ & & - & - & & $0.87(0.46 .1 .64)$ & 0.51 \\
\hline Zamora- & Spain & 40,622 & 29 & 13.6 & ICD-9 \& & Diet history/ & Hazard ratio & Anthocyanidin: & - & - & - & - & 416 & $0.96(0.88,1.03) \dagger$ & - \\
\hline Ros & (EPIC) & $(\mathrm{M} \& \mathrm{~F})$ & to 69 & & ICD-10 & Mix of & for each 2- & Total flavan-3-ol: & - & & - & - & & $0.94(0.87,1.01) \dagger$ & - \\
\hline \multirow[t]{7}{*}{$2013^{78}$} & & & & & (no codes & USDA, PE & fold $\uparrow$ in daily & Catechin: & - & & - & - & & $0.99(0.94,1.04) \dagger$ & - \\
\hline & & & & & specified) & and others & intake & PA: & - & & - & - & & $0.90(0.83,0.97) \dagger$ & - \\
\hline & & & & & & & & Flavanone: & - & & - & - & & $0.93(0.88,0.98) \dagger$ & - \\
\hline & & & & & & & & Flavone: & - & & - & - & & $0.91(0.82,1.00)^{\dagger}$ & - \\
\hline & & & & & & & & Flavonol: & - & & - & - & & $0.84(0.72,0.99) \dagger$ & - \\
\hline & & & & & & & & Isoflavone: & - & & - & - & & $1.00(0.85,1.16) \dagger$ & - \\
\hline & & & & & & & & Total: & - & & - & - & & $0.87(0.77,0.98)^{\dagger}$ & - \\
\hline Mc- & USA & 98,469 & $\sim 69$ & 7 & ICD-9 & $\mathrm{FFQ} /$ & Rate & Anthocyanidin: & $<5.5 \mathrm{vs} \geq 16.7$ & - & - & - & 2,771 & $0.86(0.76,0.97)$ & 0.04 \\
\hline Cullough & (Cancer & $(\mathrm{M} \& \mathrm{~F})$ & & & $390-459$ & USDA & & Catechin: & $<9.5 \mathrm{vs} \geq 37.2$ & & - & - & & $0.83(0.74,0.93)$ & 0.02 \\
\hline \multirow[t]{6}{*}{$2012^{54}$} & Prevention & & & & $\&$ ICD-10 & & quintile & PA: & $<71.6$ vs $\geq 253.6$ & & - & - & & $0.87(0.77,0.98)$ & 0.02 \\
\hline & Study II) & & & & I20-I99 & & & Flavanone: & $<7.0 \mathrm{vs} \geq 35.4$ & & - & - & & $0.90(0.80,1.01)$ & 0.5 \\
\hline & & & & & & & & Flavone: & $<0.5$ vs $\geq 2.1$ & & - & - & & $0.82(0.72,0.92)$ & 0.001 \\
\hline & & & & & & & & Flavonol: & $<8.5$ vs $\geq 20.6$ & & - & - & & $0.84(0.75,0.94)$ & 0.03 \\
\hline & & & & & & & & Isoflavone: & $<0.03$ vs $\geq 0.14$ & & - & - & & $0.92(0.81,1.05)$ & 0.1 \\
\hline & & & & & & & & Total: & $<121.5$ vs $\geq 359.7$ & & - & - & & $0.82(0.73,0.92)$ & 0.01 \\
\hline \multirow{6}{*}{$\begin{array}{l}\text { Mursu } \\
2008^{55}\end{array}$} & Finland & 1,950 & 42 & 15.2 & ICD-9 & 4-day & Relative & Anthocyanidin: & - & - & - & - & 102 & $0.99(0.62,1.85)$ & 0.193 \\
\hline & (Kuopio IHD & (M) & to 60 & & $390-459 \&$ & diary/USDA & risk by & Catechin + ODC: & - & & - & - & & $1.06(0.64,1.65)$ & 0.694 \\
\hline & & & & & ICD-10 & & quartiles & Flavanone: & - & & - & - & & $0.54(0.32,0.92)$ & 0.266 \\
\hline & & & & & I00-I99 & & & Flavone: & - & & - & - & & $0.65(0.40,1.05)$ & 0.333 \\
\hline & & & & & & & & Flavonol: & - & & - & - & & $1.26(0.75,2.14)$ & 0.622 \\
\hline & & & & & & & & Total: & $\overline{\mathrm{x}}=9.5$ vs $\bar{x}=435.0$ & & - & - & & $1.25(0.74,2.11)$ & 0.730 \\
\hline \multirow{5}{*}{$\begin{array}{l}\text { Kokubo } \\
2007^{101}\end{array}$} & & & 40 & 12 & ICD-10 & FFQ/ Local & & Males: & & & & & & & \\
\hline & (Japan Public & $(M \& F$ & to 59 & & I21-I23, I46, & database $^{114}$ & ratio by & Isoflavone: & $<16.4$ vs $\geq 39.6$ & 550 & $1.14(0.82,1.59)$ & 0.884 & 175 & $1.27(0.66,2.43)$ & 0.391 \\
\hline & Health Study & + post- & & & I60-I61, & & quintiles & Females: & & & & & & & \\
\hline & Cohort I) & $\begin{array}{l}\text { menopause } \\
\text { sub-group) }\end{array}$ & & & I63 \& I693 & & & $\begin{array}{l}\text { Isoflavone: } \\
\text { Post-menopause: }\end{array}$ & $<16.2$ vs $\geq 37.7$ & 226 & $0.39(0.25,0.60)$ & $<0.001$ & 57 & $0.87(0.29,2.52)$ & 0.103 \\
\hline & & & & & & & & Isoflavone: & $<16.2 \mathrm{vs} \geq 37.7$ & 148 & $0.25(0.14,0.45)$ & $<0.001$ & - & - & - \\
\hline
\end{tabular}


558 Table 1. Continued: Summary of prospective cohort studies investigating the association of flavonoid intake with total CVD incidence and mortality

\begin{tabular}{|c|c|c|c|c|c|c|c|c|c|c|c|c|c|c|c|}
\hline \multirow[b]{2}{*}{$\begin{array}{l}\text { Author, } \\
\text { year }\end{array}$} & \multirow[b]{2}{*}{$\begin{array}{l}\text { Country } \\
\text { (cohort) }\end{array}$} & \multirow[b]{2}{*}{$\begin{array}{c}n \\
\text { (population) }\end{array}$} & \multirow[b]{2}{*}{$\begin{array}{c}\text { Base- } \\
\text { line age } \\
\text { (yrs) }\end{array}$} & \multirow[b]{2}{*}{$\begin{array}{c}\text { Follow- } \\
\text { up } \\
(\mathrm{yrs})\end{array}$} & \multirow[b]{2}{*}{$\begin{array}{l}\text { Outcome } \\
\text { assessment }\end{array}$} & \multirow[b]{2}{*}{$\begin{array}{c}\text { Flavonoid } \\
\text { intake } \\
\text { estimate }^{a}\end{array}$} & \multirow[b]{2}{*}{ Analysis } & \multirow[b]{2}{*}{ Exposure $^{b}$} & \multirow[b]{2}{*}{$\begin{array}{c}\text { Intake } \\
\text { (low vs high) } \\
\text { mg/day }\end{array}$} & \multicolumn{3}{|c|}{ Total CVD incidence ${ }^{c}$} & \multicolumn{3}{|c|}{ Total CVD mortality } \\
\hline & & & & & & & & & & $n$ & $\begin{array}{c}\text { Fully adjusted } \\
\text { estimate }(95 \% \mathrm{CI}) \\
\text { [high vs low] }]^{d}\end{array}$ & $\begin{array}{l}P \text { for } \\
\text { trend }\end{array}$ & $n$ & $\begin{array}{l}\text { Fully adjusted } \\
\text { estimate }(95 \% \mathrm{Cl}) \\
\text { [high vs low] }^{d} \\
\end{array}$ & $\begin{array}{l}P \text { for } \\
\text { trend }\end{array}$ \\
\hline Mink & $\begin{array}{c}\text { USA } \\
\text {. }\end{array}$ & 34,489 & $\begin{array}{r}55 \\
\text { to } 69\end{array}$ & 16 & ICD-9 & FFQ/ & Rate ratio by & Anthocyanidin: & $<0$ vs $\geq 0.01$ & - & - & - & 2,316 & $0.91(0.83,0.99)$ & 0.032 \\
\hline $2007^{50}$ & (Iowa WHS) & (post- & to 69 & & $390-459$ & USDA & quintiles & Catechin + ODC: & $<6.8$ vs $\geq 135.7$ & & - & - & & $0.95(0.83,1.09)$ & 0.395 \\
\hline & & menopause & & & & & (except & PA: & $<89.5$ vs. $\geq 343.2$ & & - & - & & $0.93(0.81,1.07)$ & 0.332 \\
\hline & & & & & & & antho- & Flavanone: & $<16.1 \mathrm{vs} \geq 72.8$ & & - & - & & $0.88(0.77,1.01)$ & 0.054 \\
\hline & & & & & & & cyanidin & Flavone: & $<0.02$ vs $\geq 1$ & & - & - & & $0.95(0.83,1.10)$ & 0.136 \\
\hline & & & & & & & by 2 & Flavonol: & $<5.4 \mathrm{vs} \geq 14.6$ & & - & - & & $0.91(0.79,1.05)$ & 0.779 \\
\hline & & & & & & & groups) & Isoflavone: & $<0.1 \mathrm{vs} \geq 0.5$ & & - & - & & $1.05(0.91,1.21)$ & 0.210 \\
\hline & & & & & & & & Total: & $<133.2 \mathrm{vs} \geq 425.3$ & & - & - & & $0.93(0.81,1.07)$ & 0.628 \\
\hline $\begin{array}{l}\text { van der } \\
\text { Schouw }\end{array}$ & $\begin{array}{l}\text { Netherlands } \\
\text { (Prospect- }\end{array}$ & $\begin{array}{c}16,165 \\
(\mathrm{~F})\end{array}$ & $\begin{array}{l}49 \\
\text { to } 70\end{array}$ & 6.25 & $\begin{array}{c}\text { ICD-9 } \\
410-414,\end{array}$ & $\begin{array}{l}\mathrm{FFQ/Self-} \\
\text { developed }\end{array}$ & $\begin{array}{l}\text { Hazard } \\
\text { ratio by }\end{array}$ & Isoflavone: & $\quad-$ & 518 & $0.97(0.74,1.27)$ & - & - & - & - \\
\hline $2005^{102}$ & EPIC) & & & & $\begin{array}{l}427.5 \& \\
430-438\end{array}$ & database & quartiles & & & & & & & & \\
\hline $\begin{array}{l}\text { Sesso } \\
2003^{42}\end{array}$ & $\begin{array}{r}\text { USA } \\
\text { (WHS) }\end{array}$ & $\begin{array}{l}38,445 \\
(\mathrm{~F})\end{array}$ & $>45$ & 6.9 & $\begin{array}{l}\text { MI, heart } \\
\text { bypass, PTCA } \\
\text { stroke \& CVD }\end{array}$ & $\begin{array}{c}\mathrm{FFQ} / \text { Mostly } \\
\text { Hertog } e t \\
a l^{38,39}\end{array}$ & $\begin{array}{l}\text { Relative } \\
\text { risk by } \\
\text { quintiles }\end{array}$ & Flavone + flavonol: & $\tilde{x}=8.8$ vs $\tilde{x}=47.4$ & 729 & $0.88(0.68,1.14)$ & 0.63 & - & $1.05(0.62,1.78)$ & 0.12 \\
\hline
\end{tabular}

Tiet data capture method/Major flavonoid database used ${ }^{b}$ Subclass composition and calculations of flavonoid intake may differ between studies; the term 'Total' is used to denote the sum of 5 or more flavono subclasses. ${ }^{c}$ Incidence is defined as the first fatal or non-fatal event unless otherwise indicated. ${ }^{d}$ The model with the most covariate adjustments in each manuscript is reported; covariates differ between manuscripts. ${ }^{*}$ Includes Abbreviations: CAIFOS, Calcium Intake Fracture Outcome Age Related Extension Study; CHD, coronary heart disease; CVD, cardiovascular disease; EPIC, European Prospective Investigation into Cancer and Nutrition; F, female; FFQ, food frequency questionnaire; ICD, International Classification of Disease; IHD, ischemic heart disease; M, male; MI, myocardial infarction; NHS, Nurses' Health Study; ODC, other derived compounds (e.g, theaflavins and/or thearubigins); PA, proanthocyanidin; PE, Phenol-Explorer; PREDIMED, Prevención con Dieta Mediterránea; PTCA, percutaneous transluminal coronary angioplasty; SUN, Seguimiento Universidad de 


\section{b. Ischemic heart disease}

Disease overview. Globally, IHD is the main cause of death accounting for $\sim 9$ million fatalities in 2015. ${ }^{94}$ Collectively, IHD is the term for a group of clinical syndromes characterized by myocardial ischemia, most often due to atherosclerosis. ${ }^{98,115}$ Atherosclerosis can cause narrowing of the coronary arteries (stenosis) which can lead to angina pectoris, marked by paroxysms of chest pain. Secondly, atherosclerosis can cause thrombus formation in the coronary arteries, interrupting the blood supply to the heart and causing necrosis of the myocardium, in addition to several other manifestations such as coronary aneurysms. ${ }^{116} \mathrm{~A}$ growing body of mechanistic research suggests flavonoids could lower the risk of IHD by mitigating atherosclerosis and its risk factors such as hypertension, hypercholesterolemia, and endothelial dysfunction. ${ }^{79,99,100}$ Thus, the following describes findings from studies examining the association of flavonoid intake with IHD (also referred to as coronary heart disease) or its distinct disease sequelae such as myocardial infarction.

Cohort characteristics. Since 1993, at least 21 prospective cohorts (in 28 publications) have reported on flavonoid intake and the risk of IHD incidence and/or mortality (Table 2). As an aside, one other prospective study has reported on flavonoids and IHD risk, but it had an ecological study design, which differs from the other studies and is thus, not presented in table two. ${ }^{117}$ Of the publications presented, some report on the same study over different years of follow-up. For example, the Zutphen Elderly Study reports on flavone and flavonol intake and IHD risk at five and 10 years of follow-up, $, 5,118$ as well as catechin intake and IHD risk after 10 and 25 years of exposure. ${ }^{45,111}$ Other cohorts report on different classes of flavonoid intake across multiple papers, reflecting the timeline of development of several key flavonoid databases. For example, the Iowa Women's Health Study first reported on IHD risk and intake of flavones and flavonols in 1999 using tables by Hertog et al. to estimate consumption, then in 2001 on catechin intake using tables by Arts et al. to estimate exposure, then again in 2007 
using the USDA database to estimate all flavonoid subclasses and total intake; in each instance, they used updated follow-up time frames. . $^{39,46,50}$

Of the cohorts investigating flavonoid intake and IHD to date, most were conducted in Europe $(n=10)$ and the United States of America $(n=8)$ with only a minority being conducted in other parts of the world (Australia $(n=1)$ and Asia $(n=2))$. A total of 18 studies report on IHD mortality while, 19 report on IHD incidence (with nine reporting on both incidence and mortality). Nearly all studies reporting on IHD incidence defined this as the first non-fatal or fatal event. However, incidence was occasionally defined as the first non-fatal event only. ${ }^{40,76}$ Some authors presented results using both definitions (of which we present the former in Table 2). ${ }^{42}$ Approximately half of the studies reporting on incidence restricted analysis to case events of myocardial infarction. ${ }^{5,40-42,45,56,76,101,118,119}$ Studies reporting on IHD mortality tended to identify events more broadly using ICD-9 codes $410-414$, or their equivalent. Some authors presented results for total IHD as well as selected IHD subtypes, for which we present the results of the broader definition in Table 2.37 Most cohorts recruited middle aged adults, although they varied in their demographic characteristics as well as sample size (from 755 to 98,469 participants) and follow-up periods (from 4.9 to 28 years). Habitual diet was mainly assessed using FFQs ( $n=17$ publications) and the source used to estimate flavonoid exposure varied from the tables by Hertog et al. ( $n=10$ publications) to the USDA databases ( $n=7$ publications), tables by Arts et al. ( $n=3$ publications), Phenol-Explorer ( $n=2$ publications), the Singapore Composition Database ( $n=1$ publication), and several other methods $(n=5)$. All studies appear to report intake as AEs (including free form and potentially esterified flavan3-ols), except for one cohort, which reports flavonoids expressed as glycosides (and their other naturally occurring structures). ${ }^{60}$

Total flavonoids. The association between total flavonoid intake (estimated as $\geq 5$ subclasses) and IHD incidence has been studied in at least four cohorts while mortality rates have been 
studied in at least three. Of these studies, significant findings were only observed in the Danish Diet, Cancer \& Health study which examined first time events of hospitalisation for IHD (multivariable adjusted hazard ratio for highest vs lowest intake category: $0.91,95 \% \mathrm{CI}$ : 0.85 , $0.98){ }^{62}$ This study had a large sample size $(n=53,552)$, high number of cases $(n=5,323)$ and long follow-up period (23 years). While no meta-analyses have focused exclusively on total flavonoid intake and IHD, a 2017 meta-analysis pooled heterogeneous flavonoid exposures and examined their association with IHD mortality. ${ }^{106}$ Here, Liu et al. describe a trend towards a lower risk of IHD death with higher flavonoid intake ( $n=4$ cohorts; multivariable adjusted risk estimate for highest vs lowest intake category: $0.74,95 \%$ CI: $0.54,1.02 ; R=61 \%){ }^{106}$ However, these results differ to a prior meta-analysis by Jiang et al., who also pooled 4 cohorts reporting on flavonoid intake and IHD death, yet a clearly lowered risk with higher flavonoid exposure was reported (multivariable adjusted risk estimate for highest vs lowest intake category: $0.82,95 \%$ CI: $\left.0.74,0.90 ; P^{2}=22 \%\right) .{ }^{120}$ The reasons for these disparities are not clear, although may have arisen from study selection criteria. Jiang et al. further pool 15 cohorts reporting on IHD mortality $(n=4)$, IHD incidence $(n=10)$ and total CVD mortality $(n=1)$, examining any flavonoid exposure, including total intake $(n=3)$, single subclass estimates $(n$ $=5)$ or other subclass combinations $(n=7) \cdot{ }^{120}$ In this analysis, a significantly lower risk of IHD events for persons with a higher flavonoid intake was observed $(n=452,564 ; n=7,233$ cases; multivariable adjusted risk estimate for highest vs lowest intake category: $0.85,95 \% \mathrm{CI}$ : $\left.0.79,0.91, I^{2}=26 \%\right) .{ }^{120}$ Thus, while the body of evidence lends support to a beneficial association between higher flavonoid intake and IHD risk, additional research to further the granularity of evidence may be beneficial.

Flavonoid subclasses. The association between individual flavonoid subclasses and IHD incidence has been studied in at least 16 cohorts (in 19 publications), while mortality events have been studied in at least 11 cohorts (across 18 publications). Across cohorts, a lower risk of IHD tends to emerge, reaching significance in at least one or more studies, but not in more 
than half, for each individual subclass, except isoflavones for which no significant associations have been observed (Table 2). In 2003, Huxley and Neil pooled seven cohorts reporting on intakes of flavonols and risk of IHD mortality (although, some of the included studies additionally included flavones in their intake estimates). ${ }^{121}$ The authors observed a significantly lower risk of IHD mortality with higher exposures $(n=105,737$; multivariable adjusted risk estimate for highest vs lowest intake category: $0.80,95 \%$ CI: $0.69,0.93) .{ }^{121}$ More recently, Kimble et al. pooled studies reporting on intakes of anthocyanins (or berries as surrogate indicators of intake) and both IHD incidence and mortality. ${ }^{109}$ Overall, this analysis observed an association in favour of a lower risk with higher intakes and minimal publication bias was apparent ( $n=5$ studies, $n=58,638$; multivariable adjusted risk estimate for highest vs lowest intake category: $0.91,95 \%$ CI: $\left.0.83,0.99 ; 1^{2}=12.0 \%\right) .{ }^{109}$ Kimble et al. also restricted the metaanalysis to events of myocardial infarction (including incidence and mortality), yet observed no association with anthocyanin/berry intake ( $n=3$ studies, $n=175,656$; multivariable adjusted risk estimate for highest vs lowest intake category: $\left.1.00,95 \% \mathrm{CI}: 0.68,1.46,1^{2}=78.1 \%\right) .{ }^{109}$ Raman et al. reported a meta-analysis of two studies on IHD incidence and total flavan-3-ol intake finding an inverse association $(n=41,563$; multivariable adjusted risk estimate for highest vs lowest intake category: $0.81,95 \%$ CI: $0.66,0.99 ; l^{2}=60.8 \%$ ) although, no association was for observed monomer exposure ( $n=2$ studies, $n=44,514$; multivariable adjusted risk estimate for highest vs lowest intake category: $\mathrm{RR}=1.0095 \% \mathrm{CI}: 0.76,1.32) .{ }^{86}$ Seemingly, across subclasses, evidence appears stronger for anthocyanin, flavanol and flavan3-ol intake in lowering risk of IHD.

Effect modification. Modification of the relationship between flavonoid intake and IHD has been explored in a limited number of studies. Goetz et al. explored effect modification of flavonoids on IHD by age, sex, race (non-Hispanic white and black Americans), educational attainment, smoking, and physical activity in the REasons for Geographic and Racial Differences in Stroke (REGARDS) study. ${ }^{57}$ The authors enrolled 16,678 black and white males 
and females ( $\geq 45$ yrs old, $42 \%$ black, $55 \%$ female) living throughout the United States of America. ${ }^{57}$ After $6.0 \pm 1.9$ years of follow-up, 589 IHD incidences occurred however, the effect of flavonoids did not vary by the tested factors; confirmation of these findings in a larger sample would be of interest. ${ }^{57}$ Cassidy et al. examined the associations between anthocyanin intake and incidence of myocardial infarction among strata of age (participants $<65$ or $\geq 65$ years old), the presence of type 2 diabetes mellitus and hypertension, in the Health Professionals Follow-Up Study $(n=43,880) .{ }^{119}$ After 24 years of following the all-male cohort, they found no effect modification by age at baseline, nor diabetes. However the inverse association between anthocyanin intake and myocardial infarction was stronger in participants who were not hypertensive (HR: $0.81 ; 95 \%$ CI: $0.69,0.96$ ) compared to participants diagnosed with hypertension (HR: 1.05 ; 95\% CI: 0.79, 1.39; $\left.P_{\text {interaction }}=0.03\right) .{ }^{119}$ Cassidy et al. suggest this may have occurred because medication use may have exceeded the capacity for flavonoids to lower the risk of CVD. ${ }^{119}$ In the Nurses' Health Study II ( $n=93,600$; all females), Cassidy et al. also identified risk factors that may modify the relationship between flavonoid intake and myocardial infarction. ${ }^{119}$ They tested for effect modification by age, smoking, physical activity, prevalent hypertension, type 2 diabetes mellitus, alcohol intake, and body mass index. ${ }^{119}$ No significant interactions emerged. ${ }^{119}$ 
692 Table 2. Summary of prospective cohort studies investigating the association of flavonoid intake with ischemic heart disease

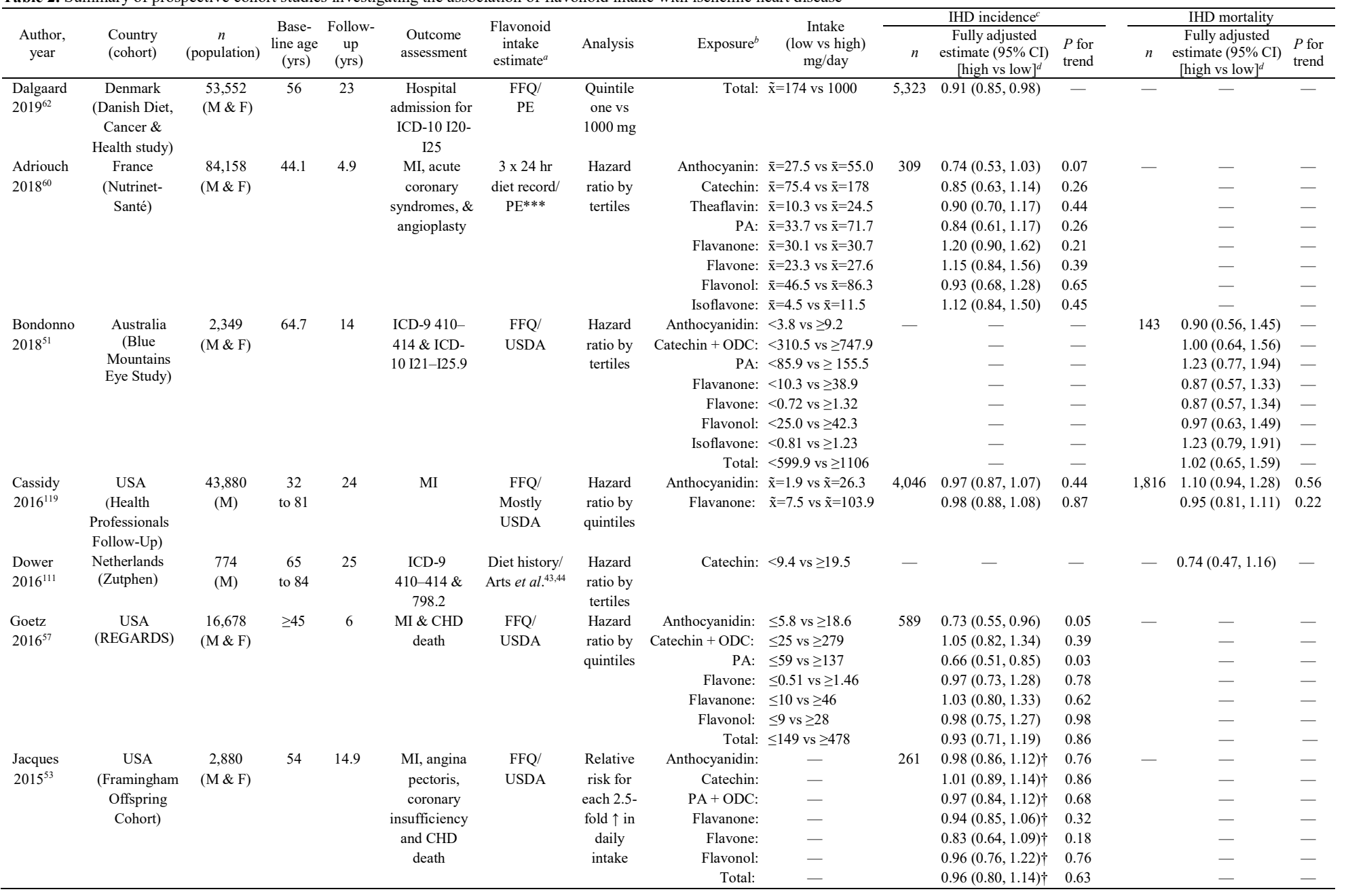


696 Table 2. Continued: Summary of prospective cohort studies investigating the association of flavonoid intake with ischemic heart disease

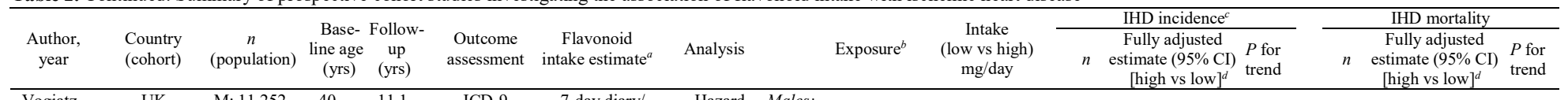

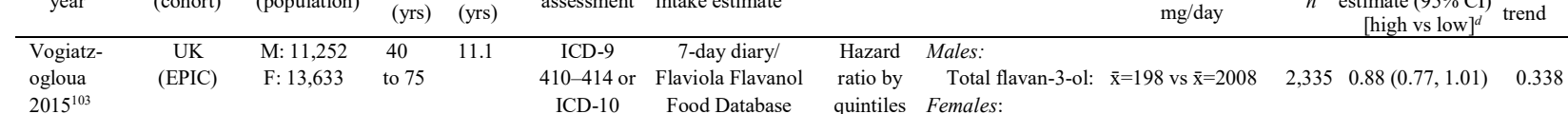

$2015^{103} \quad$ ICD-10 Food Database quintiles Females:

$\begin{array}{llllllllllll}\text { Talaei Singapore } & 63,257 & 45 & 14.7 & \begin{array}{l}\text { I20-I25 } \\ \text { ICD-9 }\end{array} & \text { FFQ/Singapore } & \text { Hazard } & \text { Total flavan-3-ol: } & \overline{\mathrm{x}}=179 \text { vs } \bar{x}=1828 & 1,325 & 0.96(0.80,1.17) & 0.810\end{array}$

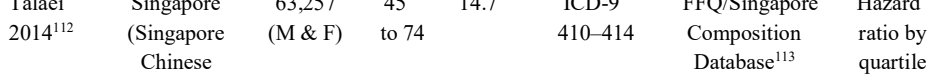
Health Study)

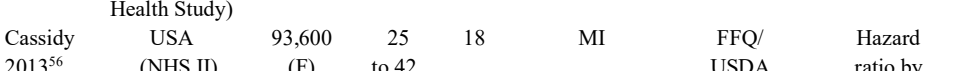

$\begin{array}{lllll}2013^{56} & \text { (NHS II) } & \text { (F) to } 42 & \text { USDA } & \begin{array}{r}\text { ratio by } \\ \text { quintiles }\end{array}\end{array}$

$$
\begin{array}{lll}
\text { Isoflavone: } & \bar{x}=5.4 \text { vs } \bar{x}=36.1
\end{array}
$$

$-\quad-\quad-$

\begin{tabular}{|c|c|c|c|c|c|c|c|c|c|c|c|c|c|c|c|}
\hline & & & & & & & & & 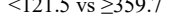 & & & & & & \\
\hline Kokubo & Japan & 40,462 & 40 & 12 & ICD-10 & FFQ/Local & Hazard & Males: & & & & & & & \\
\hline $2007^{101}$ & $\begin{array}{l}\text { (Japan PHS } \\
\text { Cohort I) }\end{array}$ & $(M \& F)$ & to 59 & & $\begin{array}{l}\mathrm{I} 21-\mathrm{I} 23 \\
\& \mathrm{I} 46\end{array}$ & database $^{114}$ & $\begin{array}{l}\text { ratio by } \\
\text { quintiles }\end{array}$ & $\begin{array}{l}\text { Isoflavone: } \\
\text { Females: }\end{array}$ & $<16.4$ vs $\geq 39.6$ & 242 & $0.77(0.47,1.24)$ & 0.614 & - & - & - \\
\hline & & & & & & & & Isoflavone: & $<16.2 \mathrm{vs} \geq 37.7$ & 66 & $0.37(0.14,0.98)$ & 0.006 & - & - & - \\
\hline Lin & USA & 66,360 & 30 & 12 & Incidence: MI & FFQ/Mostly & Relative & Flavone + & & & & & & & \\
\hline $2007^{76}$ & (NHS) & (F) & to 55 & & $\begin{array}{l}\text { Mortality: ICD } \\
-9410-414\end{array}$ & Hertog et al ${ }^{38,39}$ & $\begin{array}{l}\text { risk by } \\
\text { quintiles }\end{array}$ & flavonol: & $\leq 9.6 \mathrm{vs} \geq 30.9$ & 938 & $1.05(0.85,1.29)^{*}$ & 0.55 & 324 & $0.81(0.57,1.16)$ & 0.29 \\
\hline Mink & USA & 34,489 & 55 & 16 & ICD-9 & $\mathrm{FFQ} /$ & Rate ratio & Anthocyanidin: & 0 vs $\geq 0.01$ & - & - & - & 1,329 & $0.88(0.78,0.99)$ & 0.031 \\
\hline $2007^{50}$ & (Iowa & (post- & to 69 & & $410-414 \&$ & USDA & by quintiles & Catechin + ODC: & $<6.8$ vs $\geq 135.7$ & & - & - & & $1.02(0.86,1.21)$ & 0.113 \\
\hline & WHS) & menopau & & & 429.2 & & (except & PA: & $<89.5$ vs. $\geq 343.2$ & & - & - & & $0.91(0.76,1.10)$ & 0.489 \\
\hline & & sal & & & & & antho- & Flavanone: & $<16.1 \mathrm{vs} \geq 72.8$ & & - & - & & $0.78(0.65,0.94)$ & 0.010 \\
\hline & & women) & & & & & cyanidin & Flavone: & $<0.02$ vs $\geq 1$ & & - & - & & $0.95(0.78,1.14)$ & 0.147 \\
\hline & & & & & & & by 2 & Flavonol: & $<5.4 \mathrm{vs} \geq 14.6$ & & - & - & & $0.95(0.79,1.14)$ & 0.965 \\
\hline & & & & & & & groups) & Isoflavone: & $<0.1 \mathrm{vs} \geq 0.5$ & & - & - & & $1.00(0.83,1.19)$ & 0.425 \\
\hline & & & & & & & & Total: & $<133.2$ vs $\geq 425.3$ & & - & - & & $0.94(0.78,1.13)$ & 0.980 \\
\hline Marniemi & & 755 & 65 & 10 & & Diet history/ & Relative & Flavone + & & & & & & & \\
\hline $2005^{122}$ & $\begin{array}{l}\text { (no study } \\
\text { name) }\end{array}$ & $(M \& F)$ & to 99 & & $\begin{array}{l}410,412 \& \\
\quad 414\end{array}$ & $\begin{array}{l}\text { Nutrica } \\
\text { database }\end{array}$ & $\begin{array}{l}\text { risk by } \\
\text { tertiles }\end{array}$ & flavonol: & - & 130 & $0.79(0.51,1.24)$ & - & - & - & - \\
\hline
\end{tabular}


702 Table 2. Continued: Summary of prospective cohort studies investigating the association of flavonoid intake with ischemic heart disease

\begin{tabular}{|c|c|c|c|c|c|c|c|c|c|c|c|c|c|c|c|}
\hline \multirow[b]{2}{*}{$\begin{array}{l}\text { Author, } \\
\text { year }\end{array}$} & \multirow[b]{2}{*}{$\begin{array}{l}\text { Country } \\
\text { (cohort) }\end{array}$} & \multirow[b]{2}{*}{$\begin{array}{c}n \\
\text { (population) }\end{array}$} & \multirow[b]{2}{*}{$\begin{array}{c}\text { Base- } \\
\text { line age } \\
\text { (yrs) }\end{array}$} & \multirow[b]{2}{*}{$\begin{array}{c}\text { Follow- } \\
\text { up } \\
\text { (yrs) }\end{array}$} & \multirow[b]{2}{*}{$\begin{array}{c}\text { Outcome } \\
\text { assessment }\end{array}$} & \multirow[b]{2}{*}{$\begin{array}{c}\text { Flavonoid } \\
\text { intake } \\
\text { estimate }^{a}\end{array}$} & \multirow[b]{2}{*}{ Analysis } & \multirow[b]{2}{*}{ Exposure $^{b}$} & \multirow[b]{2}{*}{$\begin{array}{c}\text { Intake } \\
\text { (low vs high) } \\
\text { mg/day }\end{array}$} & \multicolumn{3}{|c|}{ IHD incidence $^{c}$} & \multicolumn{3}{|c|}{ IHD mortality } \\
\hline & & & & & & & & & & $n$ & $\begin{array}{c}\text { Fully adjusted } \\
\text { estimate }(95 \% \mathrm{Cl}) \text { ( }) \\
{[\text { high vs low] }}\end{array}$ & $\begin{array}{l}P \text { for } \\
\text { trend }\end{array}$ & $n$ & 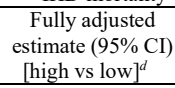 & $\begin{array}{l}P \text { for } \\
\text { trend }\end{array}$ \\
\hline $\begin{array}{l}\text { van der } \\
\text { Schouw } \\
2005^{102}\end{array}$ & $\begin{array}{c}\text { Netherlands } \\
\text { (Prospect } \\
\text { EPIC) }\end{array}$ & $\begin{array}{c}16,165 \\
\text { (F) }\end{array}$ & $\begin{array}{l}49 \\
\text { to } 70\end{array}$ & 6.25 & $\begin{array}{c}\text { ICD-9 } \\
410-414, \\
427.5\end{array}$ & $\begin{array}{l}\mathrm{FFQ} / \text { self- } \\
\text { developed } \\
\text { database }\end{array}$ & $\begin{array}{l}\text { Hazard ratio } \\
\text { by quartiles }\end{array}$ & Isoflavone: & - & 372 & $0.94(0.68,1.30)$ & - & - & - & - \\
\hline $\begin{array}{l}\text { Sesso } \\
203^{42}\end{array}$ & $\begin{array}{c}\text { USA } \\
\text { (WHS) }\end{array}$ & $\begin{array}{l}38,445 \\
\text { (F) }\end{array}$ & $>45$ & 6.9 & $\begin{array}{c}\text { MI using } \\
\text { WHO } \\
\text { criteria }\end{array}$ & $\begin{array}{l}\text { FFQ/Mostly } \\
\text { Hertog et al. } \text {. }^{88,39}\end{array}$ & $\begin{array}{l}\text { Relative risk } \\
\text { by quintiles }\end{array}$ & $\begin{array}{l}\text { Flavone + } \\
\text { flavonol: }\end{array}$ & $\tilde{\mathrm{x}}=8.8$ vs $\tilde{x}=47.4$ & - & $0.82(0.51,1.33)$ & 0.89 & - & - & - \\
\hline $\begin{array}{l}\text { Knekt } \\
2002^{47}\end{array}$ & $\begin{array}{c}\text { Finland } \\
\text { (Finnish Mobile } \\
\text { Clinic) }\end{array}$ & $\begin{array}{c}9,131 \\
(\mathrm{M} \& \mathrm{~F})\end{array}$ & 54 & 28 & $\begin{array}{c}\text { ICD-8 } \\
410-414\end{array}$ & $\begin{array}{c}\text { Diet history/ } \\
\text { Mostly local } \\
\text { analysis }\end{array}$ & $\begin{array}{l}\text { Relative risk } \\
\text { by quartiles }\end{array}$ & $\begin{array}{c}\text { Flavone }+ \\
\text { flavonol }+ \\
\text { flavanone: }\end{array}$ & - & - & - & - & 681 & $0.93(0.74,1.17)$ & 0.30 \\
\hline $\begin{array}{l}\text { Geleijnse } \\
2002^{41}\end{array}$ & $\begin{array}{l}\text { Netherlands } \\
\text { (Rotterdam } \\
\text { Study) }\end{array}$ & $\begin{array}{c}4,807 \\
(\mathrm{M} \& \mathrm{~F})\end{array}$ & $\geq 55$ & 5.6 & ICD-10 I21 & $\begin{array}{c}\mathrm{FFQ} / \\
\text { Hertog } \text { et al. }{ }^{38,39}\end{array}$ & $\begin{array}{c}\text { Relative risk } \\
\text { by tertiles }\end{array}$ & Flavonol: & $\tilde{\mathrm{x}}=16.8$ vs $\tilde{\mathrm{x}}=40.0$ & 146 & $0.76(0.49,1.18)$ & - & 30 & $0.35(0.13,0.98)$ & - \\
\hline $\begin{array}{l}\text { Arts } \\
2001^{45}\end{array}$ & $\begin{array}{l}\text { Netherlands } \\
\text { (Zutphen) }\end{array}$ & $\begin{array}{l}806 \\
\text { (M) }\end{array}$ & $\begin{array}{r}65 \\
\text { to } 84\end{array}$ & 10 & $\begin{array}{l}\text { Incidence: MI } \\
\text { Mortality: ICD } \\
-9410-414\end{array}$ & $\begin{array}{ll}\text { I } & \text { Diet history/ } \\
\text { D } & \text { Arts et al }{ }^{4,44}\end{array}$ & $\begin{array}{l}\text { Risk ratio } \\
\text { by tertiles }\end{array}$ & Catechin: & $<49.0$ vs $\geq 85.9$ & 90 & $0.49(0.27,0.88)$ & 0.017 & 90 & $0.70(0.39,1.26)$ & 0.232 \\
\hline $\begin{array}{l}\text { Arts } \\
2001^{46}\end{array}$ & $\begin{array}{c}\text { USA } \\
\text { (Iowa WHS) }\end{array}$ & $\begin{array}{l}34,492 \\
\text { (F) }\end{array}$ & $\begin{array}{r}55 \\
\text { to } 69\end{array}$ & 13 & $\begin{array}{c}\text { ICD-9 } \\
410-414 \& \\
429.2\end{array}$ & $\begin{array}{l}\text { FFQ/Mostly } \\
\text { Arts et al. } 43,44\end{array}$ & $\begin{array}{l}\text { Risk ratio by } \\
\text { quintile }\end{array}$ & Catechin: & $\tilde{\mathrm{x}}=3.7 \mathrm{vs} \tilde{\mathrm{x}}=52.7$ & - & - & - & 767 & $0.85(0.67,1.07)$ & - \\
\hline $\begin{array}{l}\text { Hertog } \\
1997^{118}\end{array}$ & $\begin{array}{l}\text { Netherlands } \\
\text { (Zutphen) }\end{array}$ & $\begin{array}{l}804 \\
(\mathrm{M})\end{array}$ & $\begin{array}{l}65 \\
\text { to } 84\end{array}$ & 10 & $\begin{array}{l}\text { Incidence: MI } \\
\text { Mortality: ICD } \\
-9410-414\end{array}$ & $\begin{array}{c}\text { Diet history/ } \\
\text { Hertog } \\
\text { et al. }]^{83,39}\end{array}$ & $\begin{array}{c}\text { Relative risk } \\
\text { by tertiles }\end{array}$ & $\begin{array}{l}\text { Flavone + } \\
\text { flavonol: }\end{array}$ & $<19$ vs $\geq 29.9$ & 92 & $0.62(0.24,1.05)$ & 0.078 & 90 & $0.47(0.27,0.82)$ & 0.006 \\
\hline $\begin{array}{l}\text { Hertog } \\
1997^{38}\end{array}$ & $\begin{array}{c}\text { UK } \\
\text { (Caerphilly } \\
\text { Study) }\end{array}$ & $\begin{array}{l}1,900 \\
(\mathrm{M})\end{array}$ & $\begin{array}{l}45 \\
\text { to } 59\end{array}$ & 14 & $\begin{array}{c}\text { MI \& IHD } \\
\text { death }\end{array}$ & $\begin{array}{c}\mathrm{FFQ} / \\
\text { Hertog et al. }\end{array}$ & $\begin{array}{l}\text { Relative risk } \\
\text { by quartiles }\end{array}$ & Flavonol: & $:<19$ vs $>34$ & 186 & $1.1(0.6,1.6)$ & 0.996 & 131 & $1.6(0.9,2.9)$ & 0.119 \\
\hline $\begin{array}{l}\text { Rimm } \\
1996^{37}\end{array}$ & $\begin{array}{c}\text { USA } \\
\text { (Health } \\
\text { Professional } \\
\text { Follow-up) }\end{array}$ & $\begin{array}{c}34,789 \\
\text { (M) }\end{array}$ & $\begin{array}{l}40 \\
\text { to } 75\end{array}$ & 6 & $\begin{array}{c}\text { MI \& } \\
\text { Revascular- } \\
\text { ization or } \\
\text { CHD death }\end{array}$ & $\begin{array}{c}\text { FFQ/Mostly } \\
\text { Hertog et al. }{ }^{88,39}\end{array}$ & $\begin{array}{l}\text { Relative risk } \\
\text { by quintiles }\end{array}$ & $\begin{array}{l}\text { Flavone + } \\
\text { flavonol: }\end{array}$ & $\tilde{x}=7.1$ vs $\tilde{x}=40.0$ & 373 & $0.94(0.68,1.31)$ & - & 140 & $0.77(0.45,1.35)$ & - \\
\hline
\end{tabular}


712 Table 2. Continued: Summary of prospective cohort studies investigating the association of flavonoid intake with ischemic heart disease

\begin{tabular}{|c|c|c|c|c|c|c|c|c|c|c|c|c|c|c|c|}
\hline \multirow[b]{2}{*}{$\begin{array}{l}\text { Author, } \\
\text { year }\end{array}$} & \multirow[b]{2}{*}{$\begin{array}{l}\text { Country } \\
\text { (cohort) }\end{array}$} & \multirow[b]{2}{*}{$\begin{array}{c}n \\
\text { (population) }\end{array}$} & \multirow[b]{2}{*}{$\begin{array}{l}\text { Base- } \\
\text { line age } \\
\text { (yrs) }\end{array}$} & \multirow[b]{2}{*}{$\begin{array}{c}\text { Follow- } \\
\text { up } \\
\text { (yrs) }\end{array}$} & \multirow[b]{2}{*}{$\begin{array}{l}\text { Outcome } \\
\text { assessment }\end{array}$} & \multirow[b]{2}{*}{$\begin{array}{c}\text { Flavonoid } \\
\text { intake } \\
\text { estimate }^{a}\end{array}$} & \multirow[b]{2}{*}{ Analysis } & \multirow[b]{2}{*}{ Exposure $^{b}$} & \multirow[b]{2}{*}{$\begin{array}{c}\text { Intake } \\
\text { (low vs high) } \\
\text { mg/day }\end{array}$} & \multicolumn{3}{|c|}{ IHD incidence ${ }^{c}$} & \multicolumn{3}{|c|}{ IHD mortality } \\
\hline & & & & & & & & & & $n$ & $\begin{array}{c}\text { Fully adjusted } \\
\text { estimate }(95 \% \mathrm{Cl} \text { ) } \\
{\left[_{\text {high vs low] }}{ }^{d}\right.}\end{array}$ & $\begin{array}{l}P \text { for } \\
\text { trend }\end{array}$ & $n$ & $\begin{array}{c}\text { Fully adjusted } \\
\text { estimate }(95 \% \mathrm{CI}) \\
\text { [high vs low] }^{d}\end{array}$ & $\begin{array}{l}P \text { for } \\
\text { trend }\end{array}$ \\
\hline $\begin{array}{l}\text { Knekt } \\
1996^{36}\end{array}$ & $\begin{array}{c}\text { Finland } \\
\text { (Finnish } \\
\text { Mobile Clinic) }\end{array}$ & $\begin{array}{l}\text { M: } 2,748 \\
\text { F: } 2,385\end{array}$ & $\begin{array}{l}30 \\
\text { to } 69\end{array}$ & 26 & $\begin{array}{c}\text { ICD-8 } \\
410-414\end{array}$ & $\begin{array}{c}\text { Diet history/ } \\
\text { Mostly } \\
\text { Hertog et all. } 3 ., 39\end{array}$ & $\begin{array}{l}\text { Relative } \\
\text { risk by } \\
\text { quartiles }\end{array}$ & $\begin{array}{l}\text { Males: } \\
\text { Flavone + flavonol: } \\
\text { Females: }\end{array}$ & $<2.1$ vs $>4.8$ & - & tho 0 & - & 324 & $0.67(0.44,1.00)$ & 0.12 \\
\hline & & & & & & & & Flavone + flavonol: & $<2.4 \mathrm{vs}>5.5$ & - & - & - & 149 & $0.73(0.41,1.32)$ & 0.21 \\
\hline $\begin{array}{l}\text { Hertog } \\
1993^{5}\end{array}$ & $\begin{array}{l}\text { Netherlands } \\
\text { (Zutphen) }\end{array}$ & $\begin{array}{l}805 \\
(\mathrm{M})\end{array}$ & $\begin{array}{l}65 \\
\text { to } 84\end{array}$ & 5 & $\begin{array}{l}\text { Incidence: MI } \\
\text { Mortality: ICD } \\
-9410-414\end{array}$ & $\begin{array}{l}\text { Diet history/ } \\
\text { Hertog et al } .^{38,35}\end{array}$ & $\begin{array}{c}\text { Relative } \\
\text { risk by } \\
\text { tertiles }\end{array}$ & $\begin{array}{r}\text { Flavone }+ \\
\text { flavonol: }\end{array}$ & $<19$ vs $\geq 29.9$ & 38 & $0.52(0.22,1.23)$ & 0.15 & 43 & $0.32(0.15,0.71)$ & 0.003 \\
\hline
\end{tabular}

${ }^{a}$ Diet data capture method/Major flavonoid database used. ${ }^{b}$ Subclass composition and calculations of flavonoid intake may differ between studies; the term 'Total' is used to denote the sum of 5 or more flavonoid subclasses. "Incidence is defined as the first fatal or non-fatal event unless otherwise indicated. ${ }^{.}$The model with the most covariate adjustments in each manuscript is reported; covariates differ between manuscripts. ${ }^{*}$ Includes Abbreviations: ATBC, Alpha-Tocopherol, Beta-Carotene Cancer Prevention Study. CHD, coronary heart disease; EPIC, European Prospective Investigation into Cancer and Nutrition; F, female; FFO food frequency questionnaire; ICD, International Classification of Disease; IHD, ischemic heart disease; M, male; MI, myocardial infarction; NHS, Nurses' Health Study; ODC, other derived compounds (e. $\mathrm{f}$, theaflavins and/or thearubigins): PA, proanthocyanidin; PE, Phenol-Explorer; PHS, Public Health Study; REGARDS, The REasons for Geographic and Racial Differences in Stroke; USA, United States of America; USDA, United States Department of Agriculture; UK, United Kingdom; WHS, Women's Health Study; WHO, World Health Organisation; $\overline{\mathrm{x}}$, mean; $\tilde{\mathrm{x}}$, median, 
Disease overview. Cerebrovascular disease is the second leading cause of death and the leading cause of adult long-term disability. ${ }^{123}$ Cerebrovascular disease is the collective term for a broad range of conditions that adversely affect cerebral blood supply including stroke, transient ischemic attacks, other intracranial vascular disorders as well as late effects of stroke or it's sequelae. ${ }^{124}$ Stroke is the primary sub-type of cerebrovascular disease and is characterised by a sudden onset of neurological dysfunction. ${ }^{125,126}$ It is typically caused by an occlusion in the cerebral vasculature resulting in an ischemic stroke and, less often, vessel rupture producing a haemorrhagic stroke. ${ }^{127}$ In high income countries, such as within North America, death from ischemic stroke accounts for as high as $67.9 \%$ of stroke mortality. ${ }^{94}$ Likewise, the prevalence of ischemic stroke dominates hemorrhagic strokes in Australasia and Western and Central Europe, reaching as high as $74.3 \%$ of stroke cases in North America. ${ }^{94}$ Vessel occlusion, occurring in ischemic stroke, is mostly a consequence of atherosclerosis, ${ }^{125}$ either locally, producing luminal narrowing precipitating obstruction, or from atherosclerotic thrombus or emboli, which form elsewhere in the body, dislodge and travel to the cerebrovasculature, where they cause significant vascular ischemia. ${ }^{126}$ As flavonoids have shown anti-atherogenic and anti-thrombotic properties, ${ }^{128}$ they are thought to play a role in mitigating the pathogenesis and progression of stroke from atherosclerotic origins. ${ }^{79,99,100}$ While most literature on flavonoid intake and cerebrovascular events has included the broad range of cerebrovascular disease in their assessment of outcome cases, as highlighted above, these are principally ASCVD related.

Cohort characteristics. At least 18 prospective cohorts (in 21 publications) have reported on flavonoid intake and risk of cerebrovascular disease incidence and/or mortality (Table 3). Some of these cohorts are reported across multiple publications, such as the Zutphen Elderly study and Iowa Women's Health Study, reflecting either the examination of certain flavonoids after different lengths of follow-up, or the examination of different flavonoids, in different 
manuscripts. Across all studies, incidence was defined as the first non-fatal or fatal event. One study restricted analyses to first time events resulting in hospitalisation..$^{62}$ Outcome events were mostly defined as all cerebrovascular disease using ICD-9 codes $430-438$ or their equivalent, to identify cases. Though, some studies reported exclusively on ischemic stroke. ${ }^{55,62,129,130}$ Others reported across multiple types of cerebrovascular disease, for which we present findings for ischemic stroke in Table 3, as flavonoids appear most relevant to ASCVD. ${ }^{35,47,119,131}$ Overall, cohorts were conducted in Europe $(n=10)$, the United States of America $(n=6)$, and Asia $(n=2)$, and they vary in size (from 552 to 98,469 participants) and follow-up periods (from 4.9 to 28 years). Flavonoid intake was mostly estimated from FFQs ( $\mathrm{n}=12$ publications) and diet histories ( $n=6$ publications) using the USDA database ( $n=6$ publications), PhenolExplorer ( $n=2$ publications), tables by Hertog et al. ( $n=3$ publications) and Arts et al. $(n=2$ publications), among a variety of other sources. Across all studies, only one indicated that flavonoid intake was estimated as glycosides (and other naturally occurring compounds); ${ }^{60}$ all other studies appear to report intake as AE (including free form and potentially esterified flavan-3-ols).

Total flavonoids. Total flavonoid intake (estimated as $\geq 5$ subclasses) has been examined in 6 cohorts for an association with cerebrovascular disease incidence or mortality. In most cohorts there was a trend towards a lower cerebrovascular disease risk with higher consumption. However, none reached statistical significance in the highest exposure category. In 2016, Tang et al. published a meta-analysis which pooled 11 cohorts $(n=356,627 ; n=5,154$ cases), reporting across incidence and mortality measures of cerebrovascular disease ( $n=8$ cohorts) and ischemic stroke ( $n=3$ cohorts), with any flavonoid exposure; from total intake ( $n=3$ cohorts), to specific subclasses or their combinations ( $n=8$ cohorts). ${ }^{132}$ In their main analysis, the authors report a significantly lower risk of cerebrovascular disease for persons with higher flavonoid intake (multivariable adjusted risk estimate for highest vs lowest intake category: 
$0.89,95 \%$ CI: $\left.0.82,0.97 ; R^{2}=0 \%\right) .{ }^{132}$ In their sub-analysis of ischemic stroke, the association was not as clear ( $n=3$ cohorts, $n=98,069$; multivariable adjusted risk estimate for highest vs lowest flavonoid intake category: $\left.0.93,95 \% \mathrm{CI}: 0.80,1.07 ; I^{2}=0 \%\right) .{ }^{132}$ However, these results are limited by the number of cohorts pooled. ${ }^{132}$ Consequently, while the body of evidence suggests an association between flavonoid intake and cerebrovascular disease risk, further research re-orientated towards disease sub-types, such as ischemic stroke, may help facilitate a better granularity of evidence.

Flavonoid subclasses. Individual flavonoid subclasses have been examined for associations with cerebrovascular disease incidence $(n=15$ cohorts across 16 publications $)$ and mortality ( $n=4$ cohorts across 6 publications). Across cohorts, a trend towards a lower risk of cerebrovascular disease emerges, reaching significance in at least one or more cohorts, for all subclasses, except flavones (Table 3). By 2010, Hollman et al. had pooled 6 cohorts $(n=$ $111,067, n=2,155$ cases), reporting intake combinations of flavonols, flavones and flavanones, and outcomes events of cerebrovascular disease and ischemic stroke, across incidence and mortality measures. ${ }^{133}$ They found, overall, a lower risk of cerebrovascular disease in those participants consuming the highest compared to the lowest estimated intakes (multivariable adjusted risk estimate: $0.80,96 \%$ CI: $0.65,0.98)$. However, heterogeneity $\left(I^{2}=54 \%\right)$ and publication bias (Eggers test $=0.01$ ) was present. ${ }^{133}$ In 2014, Wang et al . conducted an updated systematic review and meta-analysis, similar that of Hollman et al., including two further cohorts, which substantially increased the sample size and number of cases $(n=280,174, n=$ 5,228 cases). ${ }^{134}$ Again a significantly lower risk was observed (multivariable adjusted risk estimate for highest vs lowest intake category: $0.86,96 \%$ CI: $0.75,0.99)$ though, again, publication bias was indicated (Eggers test $=0.005) .{ }^{134}$ Wang et al. further conducted subanalyses, finding a trend towards a lower risk of ischemic stroke events ( $n=4$ cohorts; multivariable adjusted risk estimate for highest vs lowest intake category: $0.86,95 \%$ CI: 0.71 , 
$1.04 ; I^{2}=48 \%$ ), and unsurprisingly, given disease aetiology, this trend was less apparent for haemorrhagic stroke events ( $n=3$ cohorts; multivariable adjusted risk estimate: $0.90,95 \% \mathrm{CI}$ : $\left.0.61,1.32 ; P^{2}=50 \%\right) .{ }^{134}$ In 2019 , Kimble et al. pooled ten cohorts reporting incidence or mortality outcomes of cerebrovascular disease or specific stroke sub-types, finding no clear association with higher intake of anthocyanins or berries which were used as a surrogate indicator of anthocyanin intake (multivariable adjusted risk estimate for highest vs lowest flavonoid intake category: $1.00,95 \%$ CI: $\left.0.93,1.07 ; P^{2}=15.1 \%\right) .{ }^{109}$ When analysed by disease sub-type, there was a trend towards a lower risk of ischemic stroke with higher anthocyanin/berry consumption ( $n=4$ cohorts; multivariable adjusted risk estimate for highest vs lowest flavonoid intake category: $0.90,95 \%$ CI: $\left.0.79,1.03 ; I^{2}=0 \%\right) .{ }^{109}$ However, no such association was apparent between anthocyanin intake and haemorrhagic stroke ( $n=2$ cohorts; multivariable adjusted risk estimate for highest vs lowest flavonoid intake category: 1.01, 95\% CI: $\left.0.75,1.35 ; R^{2}=0 \%\right) .{ }^{109} \mathrm{~A}$ meta-analysis has also been performed on cohort studies reporting on flavan-3-ol intake and incident cerebrovascular disease; while no association was found, the analysis is limited by the number of included cohorts $(n=2$ cohorts; multivariable adjusted risk estimate for highest vs lowest flavonoid intake category: $0.95 ; 95 \% \mathrm{CI}: 0.80,1.12 ; I^{2}=$ $0.0 \%){ }^{86}$ However, higher flavan-3-ol monomer intake was associated with a significantly lower risk of cerebrovascular disease $(n=4$ cohorts; multivariable adjusted risk estimate for highest vs lowest flavonoid intake category: $0.82 ; 95 \%$ CI: $\left.0.68,0.99 ; I^{2}=0.0 \%\right){ }^{86}$ Thus, at this time, additional research is needed to clearly delineate the role of all flavonoid subclasses on cerebrovascular disease risk and its distinct disease sub-types.

Effect modification. The beneficial association between flavonoid-rich diets and cerebrovascular disease might differ across population subgroups. Goetz et al. evaluated 20,024 participants in the REGARDS study and tested for effect modification of flavonoids on incidence of ischemic stroke by race (non-Hispanic white and black Americans) and sex. ${ }^{129}$ 
After 6.5 years of follow-up (and 524 cases) no significant effect modification by race or sex was observed for any flavonoid exposure including all major subclasses and their total intake;

824 though corroboration of these findings in further samples is needed. ${ }^{129}$ In the Nurses' Health 825 Study $(n=69,622)$ effect modification by hypertension and aspirin use was tested $(n=1,803$ 826 cases) and no interactions were observed after 14 years of follow-up. ${ }^{131}$ In contrast, in the 827 Health Professionals Follow-Up Study, after 24 years of following 43,880 participants, significant effect modification by age occurred, wherein higher flavanone intake was associated with a lower risk of incidence ischemic stroke in men aged $>65$ years (HR: 0.71 , $95 \%$ CI: $0.54,0.92)$ compared with men $\leq 65$ y (HR: $1.0,95 \%$ CI: $0.66,1.51 ; P_{\text {interaction }}=$ $0.04),{ }^{119}$ presumably due to a higher rate of death among the more elderly. 
834 Table 3. Summary of prospective cohort studies investigating the association of flavonoid intake with cerebrovascular disease

\begin{tabular}{|c|c|c|c|c|c|c|c|c|c|c|c|c|c|c|c|}
\hline \multirow[b]{2}{*}{$\begin{array}{l}\text { Author, } \\
\text { year }\end{array}$} & \multirow{2}{*}{$\begin{array}{l}\text { Country } \\
\text { (cohort) }\end{array}$} & \multirow{2}{*}{$\begin{array}{c}n \\
\text { (population) }\end{array}$} & \multirow{2}{*}{$\begin{array}{l}\text { Base- } \\
\text { line age } \\
\text { (yrs) }\end{array}$} & \multirow{2}{*}{$\begin{array}{c}\text { Follow- } \\
\text { up } \\
\text { (yrs) }\end{array}$} & \multirow[b]{2}{*}{$\begin{array}{l}\text { Outcome } \\
\text { assessment }\end{array}$} & \multirow{2}{*}{$\begin{array}{c}\text { Flavonoid } \\
\text { intake } \\
\text { estimate }^{a}\end{array}$} & \multirow[b]{2}{*}{ Analysis } & \multirow[b]{2}{*}{ Exposure $^{b}$} & \multirow{2}{*}{$\begin{array}{c}\text { Intake } \\
\text { (low vs high) } \\
\text { mg/day }\end{array}$} & \multicolumn{3}{|c|}{$\begin{array}{l}\text { Cerebrovascular disease } \\
\text { incidence }^{c}\end{array}$} & \multicolumn{3}{|c|}{$\begin{array}{c}\text { Cerebrovascular disease } \\
\text { mortality }\end{array}$} \\
\hline & & & & & & & & & & $n$ & $\begin{array}{l}\text { Fully adjusted } \\
\text { estimate } 95 \% \text { Cl) } \\
\text { [high vs low] }]^{d}\end{array}$ & $\begin{array}{l}P \text { for } \\
\text { trend }\end{array}$ & $n$ & $\begin{array}{c}\text { Fully adjusted } \\
\text { estimate }(95 \% \mathrm{Cl}) \\
{[\text { high vs low }]^{d}}\end{array}$ & $\begin{array}{l}P \text { for } \\
\text { trend }\end{array}$ \\
\hline $\begin{array}{l}\text { Dalgaard } \\
2019^{62}\end{array}$ & $\begin{array}{c}\text { Denmark } \\
\text { (Danish Diet, } \\
\text { Cancer \& } \\
\text { Health }\end{array}$ & $\begin{array}{l}53,552 \\
(\mathrm{M} \& \mathrm{~F})\end{array}$ & 56 & 23 & $\begin{array}{l}\text { Hospital } \\
\text { admission for } \\
\text { for ICD-10 }\end{array}$ & $\begin{array}{l}\text { FFQ/ } \\
\mathrm{PE}\end{array}$ & $\begin{array}{c}\text { Quintile } \\
\text { one vs } \\
1000 \mathrm{mg}\end{array}$ & Total: & $\tilde{x}=174$ vs 1000 & 2,920 & $0.91(0.82,1.01)$ & - & - & - & - \\
\hline $\begin{array}{l}\text { Adriouch } \\
2018^{60}\end{array}$ & $\begin{array}{l}\text { France } \\
\text { (Nutrinet- } \\
\text { Santé) }\end{array}$ & $\begin{array}{c}84,158 \\
(\mathrm{M} \& \mathrm{~F})\end{array}$ & 44.1 & 4.9 & Stroke & $\begin{array}{c}3 \times 24 \mathrm{hr} \\
\text { diet record/ } \\
\mathrm{PE}^{* * *}\end{array}$ & $\begin{array}{l}\text { Hazard } \\
\text { ratio by } \\
\text { tertiles }\end{array}$ & $\begin{array}{r}\text { Anthocyanin: } \\
\text { Catechin: } \\
\text { Theaflavin: } \\
\text { PA: } \\
\text { Flavanone: } \\
\text { Flavone: } \\
\text { Flavonol: } \\
\text { Isoflavone: }\end{array}$ & $\begin{array}{l}\bar{x}=27.5 \text { vs } \bar{x}=55.0 \\
\bar{x}=7.4 \text { v } \bar{x}=178 \\
\bar{x}=1.3 \text { v } \bar{x}=24.5 \\
\bar{x}=33.7 \text { v } \bar{x}=71.7 \\
\bar{x}=30.1 \text { vs } \bar{x}=30.7 \\
\bar{x}=23.3 \text { vs } \bar{x}=27.6 \\
\bar{x}=46.5 \text { vs } \bar{x}=86.3 \\
\bar{x}=4.5 \text { vs } \bar{x}=11.5\end{array}$ & 293 & $\begin{array}{l}0.61(0.43,0.85) \\
0.69(0.51,0.93) \\
0.90(0.68,1.18) \\
0.86(0.62,1.18) \\
0.82(0.60,1.12) \\
1.09(0.79,1.50) \\
0.64(0.45,0.89) \\
0.90(0.68,1.19)\end{array}$ & $\begin{array}{l}0.007 \\
0.01 \\
0.44 \\
0.32 \\
0.25 \\
0.61 \\
0.01 \\
0.48\end{array}$ & - & $\begin{array}{l}- \\
- \\
- \\
- \\
- \\
-\end{array}$ & $\begin{array}{l}- \\
- \\
- \\
- \\
- \\
-\end{array}$ \\
\hline $\begin{array}{l}\text { Cassidy } \\
2016^{119}\end{array}$ & $\begin{array}{c}\text { USA } \\
\text { (Health } \\
\text { Professionals } \\
\text { Follow-Up) }\end{array}$ & $\begin{array}{l}43,880 \\
\text { (M) }\end{array}$ & $\begin{array}{r}32 \\
\text { to } 81\end{array}$ & 24 & $\begin{array}{l}\text { National } \\
\text { Survey of } \\
\text { Stroke criteria } \\
\text { for ischemic } \\
\text { stroke }\end{array}$ & $\begin{array}{l}\mathrm{FFQ/} \\
\text { mainly } \\
\text { USDA }\end{array}$ & $\begin{array}{l}\text { Hazard } \\
\text { ratio by } \\
\text { quintiles }\end{array}$ & $\begin{array}{l}\text { Anthocyanidins: } \\
\text { Flavanones: }\end{array}$ & $\begin{array}{c}\tilde{x}=1.9 \text { vs } \tilde{\tilde{x}}=26.3 \\
\tilde{\mathrm{x}}=7.5 \text { vs } \tilde{x}=103.9\end{array}$ & 200 & $\begin{array}{l}0.93(0.75,1.15) \\
0.78(0.62,0.97)\end{array}$ & $\begin{array}{l}0.51 \\
0.059\end{array}$ & - & 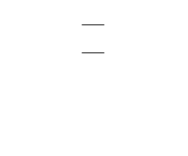 & - \\
\hline $\begin{array}{l}\text { Dower } \\
2016^{111}\end{array}$ & $\begin{array}{l}\text { Netherlands } \\
\text { (Zutphen) }\end{array}$ & $\begin{array}{l}774 \\
\text { (M) }\end{array}$ & $\begin{array}{l}65 \\
\text { to } 84\end{array}$ & 25 & $\begin{array}{c}\text { ICD-9 } \\
430-438\end{array}$ & $\begin{array}{l}\text { Diet history/ } \\
\text { Arts et al. }{ }^{43,44}\end{array}$ & $\begin{array}{l}\text { Hazard } \\
\text { ratio by } \\
\text { tertiles }\end{array}$ & Catechin: & $<9.4 \mathrm{vs} \geq 19.5$ & - & - & - & - & $0.73(0.39,1.38)$ & - \\
\hline $\begin{array}{l}\text { Vogiatz- } \\
\text { ogloua } \\
2015^{103}\end{array}$ & $\begin{array}{l}\text { UK } \\
\text { (EPIC } \\
\text { Norfolk } \\
\text { cohort) }\end{array}$ & $\begin{array}{l}\text { M: } 11,252 \\
\text { F: } 13,633\end{array}$ & $\begin{array}{l}40 \\
\text { to } 75\end{array}$ & 11.1 & $\begin{array}{c}\text { ICD-9 } \\
430-438 \\
\& \text { ICD-10 } \\
\text { I60-169 }\end{array}$ & $\begin{array}{l}\text { 7-day diary/ } \\
\text { Flaviola Flavanol } \\
\text { Food Database }\end{array}$ & $\begin{array}{l}\text { Hazard } \\
\text { 1 ratio by } \\
\text { quintiles }\end{array}$ & $\begin{array}{l}\text { Males: } \\
\text { Total flavan-3-ol: } \\
\text { Females: } \\
\text { Total flavan-3-ol: }\end{array}$ & $\overline{\mathrm{x}}=198$ vs $\overline{\mathrm{x}}=2008$ & 595 & $1.08(0.82,1.43)$ & 0.727 & - & - & - \\
\hline $\begin{array}{l}\text { Talaei } \\
2014^{112}\end{array}$ & $\begin{array}{l}\text { Singapore } \\
\text { (Singapore } \\
\text { Chinese } \\
\text { Health Study) }\end{array}$ & $\begin{array}{r}63,257 \\
(\mathrm{M} \& \mathrm{~F})\end{array}$ & $\begin{array}{l}45 \\
\text { to } 74\end{array}$ & 14.7 & $\begin{array}{c}\text { ICD-9 } \\
430-438\end{array}$ & $\begin{array}{l}\mathrm{FFQ} / \text { Singapore } \\
\text { Composition } \\
\text { Database }^{113}\end{array}$ & $\begin{array}{l}\text { Hazard } \\
\text { ratio by } \\
\text { quartile }\end{array}$ & Isoflavone: & $\overline{\mathrm{x}}=5.4$ vs $\overline{\mathrm{x}}=36.1$ & - & - & - & 1,298 & $0.97(0.81,1.16)$ & 0.41 \\
\hline
\end{tabular}


843 Table 3. Continued: Summary of prospective cohort studies investigating the association of flavonoid intake with cerebrovascular disease

\begin{tabular}{|c|c|c|c|c|c|c|c|c|c|c|c|c|c|c|c|}
\hline \multirow[b]{2}{*}{$\begin{array}{l}\text { Author, } \\
\text { year }\end{array}$} & \multirow[b]{2}{*}{$\begin{array}{l}\text { Country } \\
\text { (cohort) }\end{array}$} & \multirow[b]{2}{*}{$\begin{array}{c}n \\
\text { (population) }\end{array}$} & \multirow{2}{*}{$\begin{array}{c}\text { Base- } \\
\text { line age } \\
\text { (yrs) }\end{array}$} & \multirow{2}{*}{$\begin{array}{c}\text { Follow- } \\
\text { up } \\
\text { (yrs) }\end{array}$} & \multirow[b]{2}{*}{$\begin{array}{l}\text { Outcome } \\
\text { assessment }\end{array}$} & \multirow{2}{*}{$\begin{array}{c}\text { Flavonoid } \\
\text { intake } \\
\text { estimate }^{a}\end{array}$} & \multirow[b]{2}{*}{ Analysis } & \multirow[b]{2}{*}{ Exposure $^{b}$} & \multirow{2}{*}{$\begin{array}{c}\text { Intake } \\
\text { (low vs high) } \\
\mathrm{mg} / \text { day }\end{array}$} & \multicolumn{3}{|c|}{$\begin{array}{l}\text { Cerebrovascular disease } \\
\text { incidence }\end{array}$} & \multicolumn{3}{|c|}{$\begin{array}{c}\text { Cerebrovascular disease } \\
\text { mortality }\end{array}$} \\
\hline & & & & & & & & & & & $\begin{array}{c}\text { Fully adjusted } \\
\text { estimate }(95 \% \mathrm{CI}) \\
{[\text { high vs low] }} \\
\end{array}$ & $\begin{array}{l}P \text { for } \\
\text { trend }\end{array}$ & $n$ & $\begin{array}{l}\text { Fully adjusted } \\
\text { estimate }(95 \% \mathrm{CI}) \\
{[\text { [high vs low] }} \\
\end{array}$ & $\begin{array}{l}P \text { for } \\
\text { trend }\end{array}$ \\
\hline \multirow{5}{*}{$\begin{array}{l}\text { Cassidy } \\
2012^{131}\end{array}$} & USA & 69,622 & 30 & 14 & National & FFQ/ & Relative & Anthocyanidin: & $<5.4$ vs $>20.2$ & 943 & $30.89(0.72,1.11)$ & 0.59 & - & - & - \\
\hline & & & & & criteria for & & & Flavone: & $<0.94$ vs $>2.7$ & & $0.88(0.72,1.08)$ & 0.26 & & - & - \\
\hline & & & & & ischemic & & & Flavanone: & $<13.7$ vs $>63.0$ & & $0.81(0.66,0.99)$ & 0.10 & & - & - \\
\hline & & & & & stroke & & & Flavonol: & $<10.2 \mathrm{vs}>25.1$ & & $0.96(0.79,1.17)$ & 0.94 & & - & - \\
\hline & & & & & & & & Total: & $<150.7$ vs $>480.8$ & & $0.93(0.76,1.14)$ & 0.47 & & - & - \\
\hline \multirow{6}{*}{$\begin{array}{l}\text { Mc- } \\
\text { Cullough } \\
2012^{54}\end{array}$} & Prevention & & & & ICD-10 & & quartiles & PA: & $<71.6 \mathrm{vs} \geq 253.6$ & & - & - & & $0.88(0.70,1.10)$ & 0.8 \\
\hline & Study II) & & & & I60-I69 & & & Flavanone: & $<7.0$ vs $\geq 35.4$ & & - & - & & $0.84(0.67,1.06)$ & 0.4 \\
\hline & & & & & & & & Flavone: & $<0.5 \mathrm{vs} \geq 2.1$ & & - & - & & $0.88(0.69,1.11)$ & 0.3 \\
\hline & & & & & & & & Flavonol: & $<8.5 \mathrm{vs} \geq 20.6$ & & - & - & & $0.93(0.74,1.16)$ & 0.9 \\
\hline & & & & & & & & Isoflavone: & $<0.03$ vs $\geq 0.14$ & & - & - & & $1.00(0.78,1.28)$ & 0.8 \\
\hline & & & & & & & & Total: & $<121.5$ vs $\geq 359.7$ & & - & - & & $0.83(0.66,1.04)$ & 0.5 \\
\hline \multirow{3}{*}{$\begin{array}{l}\text { Mursu } \\
2008^{55}\end{array}$} & Finland & 1,950 & 42 & 15.2 & ICD-9 & 4-day & Relative & Anthocyanidin: & - & 102 & $0.88(0.47,1.62)$ & 0.813 & - & - & - \\
\hline & (Kuopio IHD & (M) & to 60 & & $433-434 \&$ & diary/ & risk by & Catechin + ODC: & - & & $0.59(0.30,1.14)$ & 0.102 & & - & - \\
\hline & study) & & & & ICD-10 163 & USDA & quartiles & Flavone: & - & & $1.30(0.69,2.47)$ & 0.181 & & - & - \\
\hline $\begin{array}{l}\text { Kokubo } \\
2007^{101}\end{array}$ & & & & & $\mathrm{I} 693$ & & & Isoflavone: & $<16.2 \mathrm{vs} \geq 37.7$ & 160 & $0.35(0.21,0.59)$ & 0.015 & - & - & - \\
\hline \multirow{8}{*}{$\begin{array}{l}\text { Mink } \\
2007^{50}\end{array}$} & USA & 34,489 & 55 & 16 & ICD-9 & $\mathrm{FFQ} /$ & Rate ratio & Anthocyanidin: & $<0$ vs $\geq 0.01$ & - & - & - & 469 & $1.01(0.83,1.24)$ & 0.896 \\
\hline & (Iowa WHS) & (post- & to 69 & & $430-438$ & USDA & by quintiles & Catechin + ODC: & $<6.8 \mathrm{vs} \geq 135.7$ & & - & - & & $0.95(0.71,1.28)$ & 0.724 \\
\hline & & menopausal & & & & & (except & PA: & $<89.5 \mathrm{vs} \geq 343.2$ & & - & - & & $1.02(0.74,1.39)$ & 0.983 \\
\hline & & women) & & & & & antho- & Flavanone: & $<16.1 \mathrm{vs} \geq 72.8$ & & - & - & & $0.94(0.69,1.27)$ & 0.846 \\
\hline & & & & & & & cyanidin by & Flavone: & $<0.02$ vs $\geq 1$ & & - & - & & $0.92(0.68,1.25)$ & 0.664 \\
\hline & & & & & & & 2 groups) & Flavonol: & $<5.4 \mathrm{vs} \geq 14.6$ & & - & - & & $0.92(0.67,1.24)$ & 0.984 \\
\hline & & & & & & & & Isoflavone: & $<0.1$ vs $\geq 0.5$ & & - & - & & $1.23(0.89,1.69)$ & 0.27 \\
\hline & & & & & & & & Total: & $<133.2$ vs $\geq 425.3$ & & - & - & & $0.94(0.69,1.29)$ & 0.796 \\
\hline Marniemi & Finland & & 65 & 10 & & Diet history/ & & Flavone + & & & & & & & \\
\hline $2005^{122}$ & (no name) & $(\mathrm{M} \& \mathrm{~F})$ & to 99 & & $\begin{array}{l}431,433 \\
434 \& 436\end{array}$ & Nutrica & $\begin{array}{l}\text { risk by } \\
\text { tertiles }\end{array}$ & flavonol: & - & 70 & $0.65(0.34,1.23)$ & - & - & - & - \\
\hline $\begin{array}{l}\text { van der } \\
\text { Schouw } \\
2005^{102}\end{array}$ & $\begin{array}{l}\text { Netherlands } \\
\text { (Prospect- } \\
\text { EPIC) }\end{array}$ & $\begin{array}{l}16,165 \\
(\mathrm{~F})\end{array}$ & $\begin{array}{l}49 \\
\text { to } 70\end{array}$ & 6.25 & $\begin{array}{c}\text { ICD-9 } \\
430-438\end{array}$ & $\begin{array}{l}\mathrm{FFQ/self-} \\
\text { developed } \\
\text { database }\end{array}$ & $\begin{array}{l}\text { Hazard ratio } \\
\text { by quartiles }\end{array}$ & Isoflavone: & - & 147 & $1.05(0.64,1.70)$ & - & - & - & - \\
\hline
\end{tabular}


850 Table 3. Continued: Summary of prospective cohort studies investigating the association of flavonoid intake with cerebrovascular disease

\begin{tabular}{|c|c|c|c|c|c|c|c|c|c|c|c|c|c|c|c|}
\hline \multirow[b]{2}{*}{$\begin{array}{l}\text { Author, } \\
\text { year }\end{array}$} & \multirow[b]{2}{*}{$\begin{array}{l}\text { Country } \\
\text { (cohort) }\end{array}$} & \multirow{2}{*}{$\begin{array}{c}n \\
\text { (population) }\end{array}$} & \multirow{2}{*}{$\begin{array}{c}\text { Base- } \\
\text { line age } \\
\text { (yrs) }\end{array}$} & \multirow{2}{*}{$\begin{array}{l}\text { Follow- } \\
\text { up } \\
\text { (yrs) }\end{array}$} & \multirow{2}{*}{$\begin{array}{c}\text { Outcome } \\
\text { assessment }\end{array}$} & \multirow{2}{*}{$\begin{array}{l}\text { Flavonoid } \\
\text { intake } \\
\text { estimate }^{a}\end{array}$} & \multirow[b]{2}{*}{ Analysis } & \multirow[b]{2}{*}{ Exposure $^{b}$} & \multirow{2}{*}{$\begin{array}{c}\text { Intake } \\
\text { (low vs high) } \\
\text { mg/day }\end{array}$} & \multicolumn{3}{|c|}{$\begin{array}{c}\text { Cerebrovascular disease } \\
\text { incidence }^{c}\end{array}$} & \multicolumn{3}{|c|}{$\begin{array}{c}\text { Cerebrovascular disease } \\
\text { mortality }\end{array}$} \\
\hline & & & & & & & & & & $n$ & $\begin{array}{l}\text { Fully adjusted } \\
\text { estimate (95\% CI) } \\
\text { [high vs low] }^{d}\end{array}$ & $\begin{array}{l}P \text { for } \\
\text { trend }\end{array}$ & $n$ & 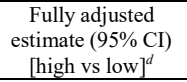 & $\begin{array}{l}P \text { for } \\
\text { trend }\end{array}$ \\
\hline $\begin{array}{l}\text { Sesso } \\
2003^{42}\end{array}$ & $\begin{array}{l}\text { USA } \\
\text { (WHS) }\end{array}$ & $\begin{array}{c}38,445 \\
(\mathrm{~F})\end{array}$ & $>45$ & 6.9 & Stroke & $\begin{array}{l}\text { FFQ/Mostly } \\
\text { Hertog } \text { et al }{ }^{38,39}\end{array}$ & $\begin{array}{l}\text { Relative } \\
\text { risk by } \\
\text { quintiles }\end{array}$ & Flavone + flavonol: & $\tilde{\mathrm{x}}=8.8$ vs $\tilde{\mathrm{x}}=47.4$ & - & $0.70(0.46,1.07)$ & 0.43 & - & - & - \\
\hline $\begin{array}{l}\text { Vokó } \\
2003^{130}\end{array}$ & $\begin{array}{l}\text { Netherlands } \\
\text { (Rotterdam } \\
\text { study) }\end{array}$ & $\begin{array}{c}5,197 \\
(\mathrm{M} \& \mathrm{~F})\end{array}$ & 67.6 & $\begin{array}{r}6.4 \\
n \\
\mathrm{n}\end{array}$ & $\begin{array}{c}\text { Review by } \\
\text { neurologist fo1 } \\
\text { schemic strok }\end{array}$ & $\begin{array}{c}\mathrm{FFQ} / \\
\text { Composite of } \\
\text { older sources }\end{array}$ & $\begin{array}{l}\text { Relative } \\
\text { risk by } \\
\text { tertiles }\end{array}$ & $\begin{array}{r}\text { Flavonoid } \\
\text { (undefined): }\end{array}$ & - & 227 & $0.79(0.66,2.12)$ & - & - & - & - \\
\hline $\begin{array}{l}\text { Knekt } \\
2002^{27}\end{array}$ & $\begin{array}{l}\text { Finland } \\
\text { (Finnish } \\
\text { Mobile } \\
\text { Clinic) }\end{array}$ & $\begin{array}{l}9,131 \\
(\mathrm{M} \& \mathrm{~F})\end{array}$ & 39.3 & 28 & $\begin{array}{c}\text { ICD-8 for } \\
\text { thrombotic } \\
\text { stroke }\end{array}$ & $\begin{array}{c}\text { Diet history/ } \\
\text { mostly local } \\
\text { analysis }\end{array}$ & $\begin{array}{l}\text { Relative } \\
\text { risk by } \\
\text { quartiles }\end{array}$ & $\begin{array}{r}\text { Flavone + flavonol } \\
+ \text { flavanone: }\end{array}$ & - & 423 & $0.73(0.54,0.98)$ & 0.004 & - & - & - \\
\hline $\begin{array}{l}\text { Arts } \\
2001^{45}\end{array}$ & $\begin{array}{l}\text { Netherlands } \\
\text { (Zutphen) }\end{array}$ & $\begin{array}{l}806 \\
\text { (M) }\end{array}$ & $\begin{array}{l}65 \\
\text { to } 84\end{array}$ & 10 & $\begin{array}{c}\text { ICD-9 } \\
430-438\end{array}$ & $\begin{array}{l}\text { Diet history/ } \\
\text { Arts et al }{ }^{43,44}\end{array}$ & $\begin{array}{l}\text { Risk ratio } \\
\text { by } \\
\text { tertiles }\end{array}$ & Catechin: & $<49.0 \mathrm{vs} \geq 85.9$ & 88 & $0.92(0.51,1.68)$ & 0.749 & 47 & $0.81(0.36,1.83)$ & 0.606 \\
\hline $\begin{array}{l}\text { Hirvonen } \\
2000^{35}\end{array}$ & $\begin{array}{l}\text { Finland } \\
\text { (ATBC } \\
\text { Study) }\end{array}$ & $\begin{array}{c}26,593 \\
\text { (M } \\
\text { smokers) }\end{array}$ & $\begin{array}{l}50 \\
\text { to } 69\end{array}$ & 6.1 & $\begin{array}{l}\text { ICD-8 for } \\
\text { cerebral } \\
\text { infarction }\end{array}$ & $\begin{array}{c}\text { Diet history/ } \\
\text { Mostly } \\
\text { Hertog et al. } l^{38,39}\end{array}$ & $\begin{array}{l}\text { Relative } \\
\text { risk by } \\
\text { quartiles }\end{array}$ & Flavone + flavonol: & $\tilde{\mathrm{x}}=4.2$ vs $\tilde{\mathrm{x}}=16.4$ & 736 & $0.98(0.80,1.21)$ & 0.81 & - & - & - \\
\hline
\end{tabular}

$851-{ }^{a}$ Diet data capture method/Major flavonoid database used. ${ }^{b}$ Subclass composition and calculations of flavonoid intake may differ between studies; the term 'Total' is used to denote the sum of 5 or more flavonoid subclasses. ${ }^{C}$ Incidence is defined as the first fatal or non-fatal event unless otherwise indicated. ${ }^{d}$ The model with the most covariate adjustments in each manuscript is reported; covariates differ between manuscripts.
$* * *$ Flavonid Nutrition; F, female; FFQ, food frequency questionnaire; ICD, International Classification of Disease; IHD, ischemic heart disease; M, male; NHS, Nurses' Health Study: ODC, other derived compounds (e. theaflanis and/or thearubigins); PA, proanthocyanidin; PE, Phenol-Explorer; PHS, Public Health Study; REGARDS, The REasons for Geographic and Racial Differences in Stroke; USA, United States of America; USDA, United States Department of Agriculture; UK, United Kingdom; WHS, Women's Health Study; $\bar{x}$, mean; $\tilde{x}$, median. 


\section{e. Peripheral artery disease}

Disease overview. It is estimated that PAD is the most prevalent CVD globally, though PAD mortality accounted for a mere $\sim 0.3 \%$ of total CVD-related death in $2015 .{ }^{94,136}$ In general PAD refers to acute or chronic obstruction of the arteries supplying the lower/upper extremities, excluding those supplying the heart or brain. ${ }^{137,138}$ The most common cause of PAD is atherosclerosis, which accounts for $>90 \%$ of cases. ${ }^{139}$ Individuals with PAD have a higher risk of coronary and cerebral atherosclerosis, and are several times more likely to experience myocardial infarction or ischemic stroke than individuals without PAD. ${ }^{140}$ The clinical spectrum of PAD is wide, including people who are asymptomatic ( $\sim 50 \%$ of those affected); to those experiencing intermittent claudication ( $\sim 10$ to $30 \%$ of PAD patients), wherein pain in the calves occurs on exercise and is relieved by rest. ${ }^{141,142}$ The most severe progression of PAD is critical limb ischemia, which develops in $\sim 1$ to $2 \%$ of PAD patients, leading to ulceration and gangrene requiring amputation in $\sim 30 \%$ of patients, and ultimately causing death in $25 \%$ of those affected within one year. ${ }^{142,143}$ Evidence suggests flavonoids attenuate pathophysiological processes involved in $\mathrm{PAD},{ }^{79,99,100}$ and consequently, we sought to report on cohorts describing the association of flavonoids with risk of PAD.

Cohort characteristics and findings. To our knowledge, only two prospective cohorts (in three publications), have investigated the association of flavonoid intake with PAD risk. As early as 2004, Hirvonen et al. evaluated the risk of intermittent claudication, after 4.1 years of followup, in 25,041 male smokers, recruited to the Alpha-Tocopherol, Beta-Carotene Cancer Prevention Study. ${ }^{144}$ The authors observed a significant inverse association in their ageadjusted, and lifestyle-adjusted models, yet, upon further adjustment for intake of vitamins C, E and carotenoids, significance was attenuated, yet a trend towards a lower risk of intermittent claudication, with higher intakes of flavonols plus flavones remained (Table 4). ${ }^{144}$ More recently, Bondonno et al. studied the risk of hospitalisation for PAD in the Danish Diet, Cancer $\&$ Health study of $>50,000$ people, over $>20$ of follow-up (in press). In their earlier manuscript,

Commented [BP1]: Reviewers to note: This article has been accepted for publication and is in press. The reference will be added and in press removed before publication of this review. 
PAD is reported as a secondary outcome, and a comparison between total flavonoid intake of $175 \mathrm{mg} /$ day (quintile one) and $1000 \mathrm{mg} /$ day is made, showing a statistical significantly lower associated risk of PAD (multivariable adjusted HR: $0.68,95 \%$ CI: $0.60,0.78$ ). ${ }^{62}$ In their latter manuscript (in press), the authors went on to study PAD in its entirety. In the same cohort, after adjustment for lifestyle and dietary factors, a lower risk of PAD was observed, across all subclasses, reaching significance for intake of anthocyanin, catechins, proanthocyanidins, flavonols and total flavonoids (Table 4). The non-linear association between total flavonoid intake and PAD hospitalizations plateaued at $\sim 750$ to $1000 \mathrm{mg} /$ day after which no added benefit was observed. Moreover, across secondary endpoints, compared to a total flavonoid intake of $174 \mathrm{mg} /$ day, for intakes of $1000 \mathrm{mg} /$ day and $500 \mathrm{mg} /$ day respectively, a lower risk of revascularizations/endovascular surgery [HR: 0.57 (95\% CI 0.46, 0.69)], and amputations [HR: $0.56(95 \%$ CI $0.43,0.74)]$ was observed. Furthermore, on both a relative and an absolute scale, the greatest benefits observed for higher flavonoid intakes were in current or former smokers. Overall, evidence is sparse yet promising, that higher intake of flavonoid rich foods lowers risk of PAD. 
904 Table 4. Summary of prospective cohort studies investigating the association of flavonoid intake with peripheral artery disease

\begin{tabular}{|c|c|c|c|c|c|c|c|c|c|c|c|c|c|c|c|}
\hline \multirow[b]{2}{*}{$\begin{array}{l}\text { Author, } \\
\text { year }\end{array}$} & \multirow[b]{2}{*}{$\begin{array}{l}\text { Country } \\
\text { (cohort) }\end{array}$} & \multirow[b]{2}{*}{$\begin{array}{c}n \\
\text { (population) }\end{array}$} & \multirow[b]{2}{*}{$\begin{array}{l}\text { Base- } \\
\text { line age } \\
\text { (yrs) }\end{array}$} & \multirow[b]{2}{*}{$\begin{array}{c}\text { Follow- } \\
\text { up } \\
\text { (yrs) }\end{array}$} & \multirow[b]{2}{*}{$\begin{array}{l}\text { Outcome } \\
\text { assessment }\end{array}$} & \multirow[b]{2}{*}{$\begin{array}{c}\text { Flavonoid } \\
\text { intake } \\
\text { estimate }^{a}\end{array}$} & \multirow[b]{2}{*}{ Analysis } & \multirow[b]{2}{*}{ Exposure $^{b}$} & \multirow[b]{2}{*}{$\begin{array}{c}\text { Intake } \\
\text { (low vs high) } \\
\text { mg/day }\end{array}$} & \multicolumn{3}{|c|}{ PAD incidence ${ }^{c}$} & \multicolumn{3}{|c|}{ PAD mortality } \\
\hline & & & & & & & & & & $n$ & $\begin{array}{l}\text { Fully adjusted } \\
\text { estimate }(95 \% \mathrm{CI}) \\
{[\text { [high vs low] }}\end{array}$ & $\begin{array}{l}P \text { for } \\
\text { trend }\end{array}$ & $n$ & 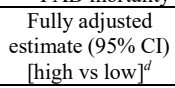 & $\begin{array}{l}P \text { for } \\
\text { trend }\end{array}$ \\
\hline $\begin{array}{l}\text { Bondonno } \\
2020\end{array}$ & $\begin{array}{c}\text { Denmark } \\
\text { (Danish Diet, }\end{array}$ & $\begin{array}{c}56,048 \\
(\mathrm{M} \& \mathrm{~F})\end{array}$ & 56 & 23 & $\begin{array}{c}\text { Hospital } \\
\text { admission for }\end{array}$ & $\begin{array}{c}\mathrm{FFQ} / \\
\mathrm{PE}\end{array}$ & $\begin{array}{l}\text { Hazard } \\
\text { ratio by }\end{array}$ & $\begin{array}{l}\text { Anthocyanidin: } \\
\text { Catechin: }\end{array}$ & $\begin{array}{l}<10 \text { vs } \geq 53 \\
<21 \text { vs } \geq 282\end{array}$ & 2,131 & $\begin{array}{l}10.86(0.76,0.98) \\
0.80(0.71,0.91)\end{array}$ & - & - & - & - \\
\hline & $\begin{array}{c}\text { Cancer \& } \\
\text { Health study) }\end{array}$ & & & & $\begin{array}{l}\text { ICD-10 I70- } \\
\text { I74 }\end{array}$ & & quintiles & $\begin{array}{r}\text { PA t theaflavin: } \\
\text { Flavanone: } \\
\text { Flavone: } \\
\text { Flavonol: } \\
\text { Total: }\end{array}$ & $\begin{array}{l}<136 \text { vs } \geq 434 \\
<6 \text { vs } \geq 49 \\
<3 \text { vs } \geq 9 \\
<21 \text { vs } \geq 83 \\
<251 \text { vs } \geq 909\end{array}$ & & $\begin{array}{l}0.69(0.60,0.80) \\
0.95(0.83,1.09) \\
0.88(0.76,1.02) \\
0.73(0.63,0.83) \\
0.75(0.65,0.86)\end{array}$ & & & & \\
\hline $\begin{array}{l}\text { Dalgaard } \\
2019^{62}\end{array}$ & $\begin{array}{c}\text { Denmark } \\
\text { (Danish Diet, } \\
\text { Cancer \& } \\
\text { Health study) }\end{array}$ & $\begin{array}{l}53,552 \\
(\mathrm{M} \& \mathrm{~F})\end{array}$ & 56 & 23 & $\begin{array}{c}\text { Hospital } \\
\text { admission for } \\
\text { ICD-10 I70- } \\
\text { I74 }\end{array}$ & $\begin{array}{c}\mathrm{FFQ} / \\
\mathrm{PE}\end{array}$ & $\begin{array}{c}\text { Quintile } \\
\text { one vs } \\
1000 \mathrm{mg}\end{array}$ & Total: & $\tilde{\mathrm{x}}=174$ vs 1000 & 1,867 & $0.68(0.60,0.78)$ & - & - & - & - \\
\hline $\begin{array}{l}\text { Hirvonen } \\
2004^{144}\end{array}$ & $\begin{array}{c}\text { Finland } \\
\text { (ATBC Study) }\end{array}$ & $\begin{array}{c}25,041 \\
(\mathrm{M} \\
\text { smokers) }\end{array}$ & $\begin{array}{c}50 \\
\text { to } 69\end{array}$ & 4.1 & $\begin{array}{l}\text { IC using Rose } \\
\text { questionnaire }\end{array}$ & $\begin{array}{l}\text { Diet history/ } \\
\text { Mostly Hertog } \\
\text { et al. }{ }^{38,39}\end{array}$ & $\begin{array}{c}\text { Relative } \\
\text { risk by } \\
\text { quintiles }\end{array}$ & Flavone + flavonol: & - & 2,412 & $0.93(0.81,1.08)$ & 0.12 & - & - & - \\
\hline
\end{tabular}

905 a ${ }^{a}$ Diet data capture method/Major flavonoid database used. ${ }^{b}$ Subclass composition and calculations of flavonoid intake may differ between studies; the term 'Total' is used to denote the sum of 5 or more flavonoid 906 subclasses. ${ }^{c}$ Incidence is defined as the first fatal or non-fatal event unless otherwise indicated. ${ }^{d}$ The model with the most covariate adjustments in each manuscript is reported; covariates differ between manuscripts.
Commented [BP2]: Reviewers to note: This article has been accepted for publication and is in press. The reference will be added and in press removed before publication of this correct as: "27 prospective cohorts in $\mathbf{4 4}$ publications" 


\section{Discussion: current evidence—future epidemiological frontiers}

In the absence of long-term RCTs, the prospective cohort studies conducted to date offer critical information on the typical foods and beverages consumed by populations, their relative flavonoid contents, and the long-term association with CVD incidence and mortality. Over the past three decades, at least 27 prospective cohorts (in 44 publications) have evaluated the relationship between estimated flavonoid intake and CVD risk. While inconsistencies in findings are observed between studies, meta-analyses of these cohorts tend to suggest a common significant association, wherein, habitual, long-term intakes of flavonoid-rich diets may be associated with a lower risk of IHD, cerebrovascular disease, and total CVD. Less evidence is available for PAD. Of the flavonoid subclasses investigated, epidemiological evidence more often supports diets rich in anthocyanins, flavan-3-ols, and flavonols in lowering the risk of chronic CVD. At the same time, several mechanisms have been investigated by which flavonoids might modulate the causal pathway of major CVD. To this end, short-term RCTs provide evidence that flavonoids lower blood pressure, ${ }^{86}$ improve endothelial function, ${ }^{86}$ improve arterial stiffness, ${ }^{82}$ reduce dyslipidaemia, ${ }^{86}$ and mediate inflammatory responses. ${ }^{145}$ As these mechanisms are collectively implicated in atherosclerosis, they are thought, among other possible mechanisms, to conjointly influence the trajectory of chronic CVD, particularly, ASCVD. ${ }^{79,99,100}$ Seemingly, converging evidence of the effects of flavonoids on intermediate endpoints and their association with hard clinical outcomes is suggestive that flavonoid-rich foods, may be protective against CVD. If confirmed, food policy strategies incorporating evidence of the benefits of flavonoid-rich diets may have important implications for health care services and public health outcomes.

While meta-analyses tend to suggest a significant inverse association between flavonoid intake CVD risk, some inconsistencies in findings between the cohort studies seem apparent. There are multiple potential reasons for these discrepancies, including differences in demographic 
characteristics or study settings, differences in the actual absolute intake between populations, the length of follow-up, the varying definition of outcomes, accuracy of covariate assessment, heterogeneous covariate adjustments, the sample size (and number of cases) and consequently, adequate statistical power for meaningful conclusions. ${ }^{146}$ Moreover, U-shaped associations have been seen, wherein participants with moderate intakes have a significantly lower risk of CVD, but for those in the highest intake category, this association is not significant. The reason for this finding is not entirely clear, but fewer individuals with these high intakes means less certainty (wider confidence intervals), and findings must be interpreted with caution. In addition, the studies used different flavonoid databases at varying stages of their 'development' which may have also contributed to varying outcomes; to this end, the known limitations of using databases to calculate dietary intake also apply. ${ }^{13,68}$ Further, dietary assessment tools also contribute to measurement error in the exposure. ${ }^{13}$ Many of the studies summarised in this review used FFQs, and many of these FFQs have not been validated specifically for flavonoids. Though, even if they were, such self-reported dietary data would still contain measurement error and this has long been acknlowegded. ${ }^{147,148}$ Such uncertainty in exposure data translates into uncertainty of outcomes and biases associations towards the null raising the possibility of false-negatives. Thus, null findings may not necessarily predicate no association. Indeed, in meta-analyses of prospective cohorts, although often accompanied by statistically significant heterogeneity (likely from some of the aforementioned causes), an overall link between flavonoid-rich diets and IHD, cerebrovascular disease and total-CVD emerges.

As the temporal relationship between the exposures and outcomes reveals that reverse causality is unlikely attributable, this supports the presence of a beneficial association in favour of flavonoid intake. However, whether the associations represent a benefit of the isolated bioactives per se, or a signal of the bioactives working in concert with the co-occurring nutrient matrix within flavonoid-bearing foods, are issues of consideration..$^{4,149-152}$ Moreover, whether beneficial associations between total flavonoid intake and CVD is driven by flavan-3-ols, the 
principal subclass consumed in most populations, or whether it represents the breakdown of several subclasses into common downstream bioactive metabolites, must be considered. Even the biological rationale for examining individual subclasses is somewhat tenuous as differences in the bioavailability and bioactivity of individual compounds within the same subclass is observed. Indeed, a central limitation of quantifying flavonoid intake based on aglycones is that the varying effects of different glycosides ${ }^{10}$ cannot be taken into consideration; this is best investigated in clinical trials and animal studies. Thus, while the calculation and correlation of aglycones with endpoints might be useful to obtain a broad overview of the relationships, there are several caveats with this approach. Thus, the simple interpretation of the data to date, and the one most relevant for dietary advice, is that consumption of flavonoid-rich foods or diets higher in flavonoids, appear nutritionally beneficial in the prevention of CVD.

Although, if persons with higher flavonoid intakes tend to have other positive dietary habits, ${ }^{61}$ one may question whether associations observed can be attributable to flavonoid-rich foods or whether these diets are simply a marker of higher diet quality overall. To address this issues, one approach has been to add potential dietary confounders as covariates in multivariable adjusted models, or to adjust for a "diet-quality" index. While the former approach is sensible when the dietary confounders are not also a source of flavonoids, risk estimates arising from models adjusting for flavonoid sources, such as fruits and vegetables, become difficult to interpret. Using a different approach, Bondonno et al. report evidence of an association between total flavonoid intake and all-cause mortality amongst participants in the highest fruit and vegetable intake tertile, suggesting that there may be a benefit to recommending a diet rich in flavonoids, above and beyond a diet rich in fruits and vegetables. ${ }^{61}$ This is reasonable as it is possible to have a diet low in fruits and vegetables but high in flavonoids and vice versa. Thus, the observed associations may not necessarily be a mere marker of higher overall diet quality, although, we are unable to rule out this hypothesis. Yet, to this end, we postulate that 
higher intakes of flavonoid-rich foods are likely one factor of a healthy diet, which when coupled with other beneficial dietary habits, are likely to produce the greatest health benefits.

Comprehensive knowledge on the current state of the science is a prerequisite for hypothesis formulation and the design and conduct of further research. This review traces the development and advances of flavonoid epidemiology providing a broad overview and update on the current state of the science. To the best of our knowledge we include all major studies (written in English) and our general conclusions are in alignment with other reviews in the field, however, we limit our scope to intake of total flavonoids and major subclasses and selected CVD outcomes. To this end, a growing body of studies have also addressed individual flavonoid compounds and their relationship to CVD. ${ }^{61,153}$ These compounds may warrant further research, where intake is sufficiently variable and at potentially biologically relevant quantities in the populations being studied. To this end, a growing body of cohort studies have also recently addressed flavonoid intake and other CVDs or related risk factors, including, yet not limited to, risk of hypertension, ${ }^{154-156}$ type 2 diabetes, ${ }^{157}$ and overweight and obesity, ${ }^{158,159}$ which in general, show a protective association across such non-communicable conditions related to CVD.

In considering the design of further research, careful selection of lifestyle confounders is an issue of importance. This is because dietary intake may relate in a complicated manner to other social and behavioural determinants of disease. To date, most investigators have adjusted for age, sex (in mixed cohorts), body mass index, physical activity, and smoking history while, sometimes, indices of cardio-metabolic health, such as blood pressure, cholesterol and/or diabetes were incorporated. Not all studies adjusted for indices of socioeconomic status (SES), be it via education, income or profession. As flavonoid intake appears to be associated with SES, which is in turn associated with $\mathrm{CVD},{ }^{160}$ there is reason to consider adjustment for SES in future studies. Though of course, confounders within cohorts will depend on the specific 
population recruited. Most authors adjusted for total energy intake, via either a standard multivariable model or another method (e.g. nutrient residual). ${ }^{161}$ However some authors presented models without energy adjustment, as it is thought that absolute consumption (adjusted for body size), may be more relevant than energy-adjusted values. ${ }^{51,61,62}$ Although these studies tended to additionally conduct sensitivity analysis with energy included. Adjusting for energy intake made no difference, however, the interpretation of the result changes. Considerable heterogeneity is found in the current literature with regards to the adjustment of other dietary factor, with many authors not considering dietary cofounders at all. Thus, residual confounding in this regard likely to vary between studies.

Several knowledge gaps exist in the current evidence base; such gaps may need to be addressed if flavonoid epidemiology research is to inform dietary guidelines. ${ }^{70,162,163}$ There are only a few studies available on flavonoid intake and ischemic stroke as well as PAD and, as such, there is a need for further study in these areas. Moreover, although included under the umbrella of total CVD, flavonoid intake does not appear to be associated with atrial fibrillation, ${ }^{164}$ or haemorrhagic stroke; ${ }^{134}$ investigations targeting total ASCVD may be more appropriate than all-cause CVD, as flavonoids appear most relevant to CVDs with atherosclerotic origins. Including atrial fibrillation, which has a relatively high prevalence, ${ }^{165}$ within total CVD outcomes plausibly dilutes the association with flavonoid intake. Findings from the Danish Diet Cancer and Health Study cohort also suggest that a high flavonoid intake may be most beneficial to populations at risk of CVD, namely smokers and high alcohol consumers. ${ }^{61,62}$ Although similar effect modification by smoking or alcohol intake was not observed in the Nurses' Health Study II or the REGARDS cohort; ${ }^{57,119}$ these analyses were specifically conducted in relation to IHD in contrast to total atherosclerotic CVD which was looked at in the Danish Diet Cancer and Health Study. A limitation of the Danish cohort is that the Danish population is more homogenous than many other countries, with most of the participants being Caucasian, limiting the generalizability of the findings to other ethnicities. Thus, further studies 
1042 are warranted to determine whether similar associations exist in other ethnic groups. As the 1043 inverse association between flavonoid intake and CVD may be stronger in subgroups of 1044 smokers and high alcohol consumers, the associations between flavonoid intake and CVD may differ in cohorts that have a higher proportion of these individuals. To this end, modification of the flavonoid-CVD association by other potential effect modifiers has been explored in a limited number of cohorts. No effect modification was found by sex, race, physical activity, education, aspirin use, type 2 diabetes mellitus, or body mass index, however, at least one study reported effect modification by age. ${ }^{57,61,119,129,131}$ As a result, it is also likely that the associations between flavonoid intake and CVD may appear stronger in cohorts that have a higher proportion of older individuals. Other differences which may also affect the association, such as length of follow-up or geographical region, are of interest. However, a recent metaanalysis found no difference in results, when subgroup analysis was performed on studies with these differing attributes (i.e., studies with less or greater than 10 years of follow-up and Mediterranean compared to non-Mediterranean countries) however, the number of studies included was small $(\mathrm{n}=5) \cdot{ }^{105}$ Corroboration of these findings in further samples is needed as is the examination of other potential effect modifiers.

In the context of advancing flavonoid research, the prospective cohort study appears to be the most feasible design realistically available for examining dietary exposures with long-term 1060 disease outcomes. ${ }^{70,166,167}$ Though of course, large-scale RCTs, where-ever viable, would 1061 immensely facilitate progress in this field. One long-awaited RCT, involving $>20,000$ 1062 participants, is the COcoa Supplement and Multivitamin Outcomes Study, which aims to examine the effect of flavan-3-ol intake on CVD outcomes, over 5 years of follow-up. ${ }^{168}$ Moving forward, the use of nutritional biomarkers or other innovative measures of flavonoid intake, in both RCTs and observational studies, provides a major future pathway for strengthening the investigation of flavonoids, by providing objective assessments of dietary intake or, in the case of biomarkers, further allowing for calibrated adjustment of subjective 
intake values. ${ }^{169-171}$ There is a crucial need for quality biomarkers of intake as a means of overcoming well-known FFQ and food database limitations. ${ }^{172}$ As well as being subjective and restricted, the use of FFQs to estimate flavonoid exposure does not allow for differences in metabolism between individuals to be taken into consideration. This is crucial as metabolism affects the levels of circulating bioactive downstream metabolites and may be key in explaining why some individuals benefit from the consumption of flavonoid-rich foods whilst others do not. Furthermore, many cohort studies do not have FFQ data. Indeed, several recent metaanalyses of prospective cohorts using (poly)phenol biomarkers and assessing CVD outcomes, have shown a paucity of studies using this approach. ${ }^{173,174}$ With the ever-growing progress in identifying biomarkers of flavonoid exposure, ${ }^{68,170}$ this approach is likely to usher in a new era of research, with a combination of dietary data and biomarkers likely being the most favourable approach. One recent example is a case-control study by Murphy et al., whom used a newly developed method for quantifying 38 dietary (poly)phenols in plasma-seemingly the most comprehensive method for (poly)phenol biomarker assessment developed to date. ${ }^{175,176}$ Another important advantage of using biomarkers is their potential ability to capture exposure to foods and/or flavonoids, not included in food composition databases, such as food additives or dietary supplements. ${ }^{167}$ However, biomarkers are not without their own limitations and readers are directed to several key reviews for further discussion. ${ }^{68,170,171}$

Future studies addressing several other limitations may also provide a more robust basis for evaluating the association between flavonoid intake and CVD. In prospective cohort studies lasting many years, repeated measures of dietary intake and other covariates would allow for cumulative exposures to be included, reducing regression dilution bias resulting from measurement error, and increasing confidence in the findings. One example can be seen in the Nurses' Health Study II by Cassidy et al., where FFQs were collected every 4 years (as well as covariate data biennially) providing better estimates of average habitual flavonoid exposure (and covariate status) over the 18 year follow-up timeframe. ${ }^{56} 56$ Other improvements include 
increasing the reliability of the evidence base. Given the rising threat of the reproducibility crisis, cherry-picking and p-hacking, ${ }^{177-179}$ meta-analysis authors are encouraged to test for bias across all exposure-outcome assessments and are advised to include technical flavonoid experts on their team. While results of meta-analyses to date generally indicate that publication bias appears less prevalent in flavonoid research, both Hollman et al., ${ }^{133}$ and Wang et al., ${ }^{134}$ found evidence of publication bias, and thus caution is needed in interpreting the results on cerebrovascular disease. To facilitate improvements, epidemiologists are urged to adopt reporting guidelines for cohort studies, ${ }^{180}$ and flavonoid-specific research, ${ }^{181}$ as well as consider open access databases, and the pre-registration of analysis plans for cohort studies, thereby improving transparency and the reproducibility of research practices. ${ }^{182}$

\section{Conclusion}

Nutrition plays a critical role in preserving cardiovascular health and lowering the risk of major CVD. In the early 1990's, prospective studies began to emerge, reporting beneficial associations between a higher dietary flavonoid intake and a lower risk of CVD. Since this time

1108 a large volume of literature has accumulated. During the last $\sim 25$ years, the completeness of

1109 flavonoid-food composition databases have advanced substantially, although this brings with

1110 it inherent difficulty when cross-comparing (old and new) studies. This could explain some of

1111 the discrepancies between cohort studies, however difference in other cohort characteristics have likely also contributed. Differences in outcome definitions and the inclusion of total CVD cases, rather than those specifically of ASCVD, may have attenuated results, based on what is known on proposed flavonoid mechanism and atherosclerosis. Despite these limitations, the evidence from prospective cohort studies, when combined in meta-analyses, suggests, habitual,

1116 long-term moderate consumption of flavonoid-rich diets may be associated with lower risk of

1117 CVD. Specifically, the totality of evidence suggests a protective association between flavonoid

1118 intake and IHD, cerebrovascular disease and total CVD; disease outcomes which are 
1119 principally, though not exclusively, composed of cases of ASCVD. Limited available evidence

1120 also suggests a role of flavonoids in mitigating the risk of PAD and ischemic stroke although,

1121 further research is required. Of the subclasses investigated, evidence for a protective

1122 association appears strongest for diets rich in anthocyanin, flavan-3-ol, and flavonol intake. If

1123 confirmed, these findings have important clinical and public health implications as flavonoids

1124 are common in foods and CVD is of major public health concern. A major future pathway in

1125 studies of flavonoid intake is the use of nutritional biomarkers, in combination with traditional

1126 food intake approaches. Future epidemiological research re-orientated towards the

1127 investigation of ASCVD may enhance the evidence base as flavonoids are primarily

1128 hypothesised to lower the risk of CVD of atherosclerotic origins.

1129

1130

1131

1132

1133

1134

1135

1136

1137 
11441 S. S. Lim, T. Vos, A. D. Flaxman, G. Danaei, K. Shibuya, H. Adair-Rohani, M. Amann, H. R. Anderson, K. G. Andrews, M. Aryee, C. Atkinson, L. J. Bacchus, A. N. Bahalim, K. Balakrishnan, J. Balmes, S. Barker-Collo, A. Baxter, M. L. Bell, J. D. Blore, F. Blyth, C. Bonner, G. Borges, R. Bourne, M. Boussinesq, M. Brauer, P. Brooks, N. G. Bruce, B. Brunekreef, C. Bryan-Hancock, C. Bucello, R. Buchbinder, F. Bull, R. T. Burnett, T. E. Byers, B. Calabria, J. Carapetis, E. Carnahan, Z. Chafe, F. Charlson, H. Chen, J. S. Chen, A. T. A. Cheng, J. C. Child, A. Cohen, K. E. Colson, B. C. Cowie, S. Darby, S. Darling, A. Davis, L. Degenhardt, F. Dentener, D. C. Des Jarlais, K. Devries, M. Dherani, E. L. Ding, E. R. Dorsey, T. Driscoll, K. Edmond, S. E. Ali, R. E. Engell, P. J. Erwin, S. Fahimi, G. Falder, F. Farzadfar, A. Ferrari, M. M. Finucane, S. Flaxman, F. G. R. Fowkes, G. Freedman, M. K. Freeman, E. Gakidou, S. Ghosh, E. Giovannucci, G. Gmel, K. Graham, R. Grainger, B. Grant, D. Gunnell, H. Hubbell, S. J. Hutchings, S. E. Ibeanusi, G. L. Jacklyn, R. Jasrasaria, J. B. Jonas, H. Kan, J. A. Kanis, N. Kassebaum, N. Kawakami, Y. H. Khang, S. Khatibzadeh, J. P. Khoo, C. Kok, F. Laden, R. Lalloo, Q. Lan, T. Lathlean, J. L. Leasher, J. Leigh, Y. Li, J. K. Lin, S. E. Lipshultz, S. London, R. Lozano, Y. Lu, J. Mak, R. Malekzadeh, L. Mallinger, W. Marcenes, L. March, R. Marks, R. Martin, P. McGale, J. McGrath, S. Mehta, G. A. Mensah, T. R. Merriman, R. Micha, C. Michaud, V. Mishra, K. M. Hanafiah, A. A. Mokdad, L. Morawska, D. Mozaffarian, T. Murphy, M. Naghavi, B. Neal, P. K. Nelson, J. M. Nolla, R. Norman, C. Olives, S. B. Omer, J. Orchard, R. Osborne, B. Ostro, A. Page, K. D. Pandey, C. D. H. Parry, E. Passmore, J. Patra, N. 
Pearce, P. M. Pelizzari, M. Petzold, M. R. Phillips, D. Pope, C. A. Pope, J. Powles, M. Rao, H. Razavi, E. A. Rehfuess, J. T. Rehm, B. Ritz, F. P. Rivara, T. Roberts, C. Robinson, J. A. Rodriguez-Portales, I. Romieu, R. Room, L. C. Rosenfeld, A. Roy, L. Rushton, J. A. Salomon, U. Sampson, L. Sanchez-Riera, E. Sanman, A. Sapkota, S. Seedat, P. Shi, K. Shield, R. Shivakoti, G. M. Singh, D. A. Sleet, E. Smith, K. R. Smith, N. J. C. Stapelberg, K. Steenland, H. Stöckl, L. J. Stovner, K. Straif, L. Straney, G. D. Thurston, J. H. Tran, R. Van Dingenen, A. Van Donkelaar, J. L. Veerman, L. Vijayakumar, R. Weintraub, M. M. Weissman, R. A. White, H. Whiteford, S. T. Wiersma, J. D. Wilkinson, H. C. Williams, W. Williams, N. Wilson, A. D. Woolf, P. Yip, J. M. Zielinski, A. D. Lopez, C. J. L. Murray and M. Ezzati, A comparative risk assessment of burden of disease and injury attributable to 67 risk factors and risk factor clusters in 21 regions, 1990-2010: A systematic analysis for the Global Burden of Disease Study 2010, Lancet, 2012, 380, 2224-2260.

A. Afshin, P. J. Sur, K. A. Fay, L. Cornaby, G. Ferrara, J. S. Salama, E. C. Mullany, K. H. Abate, C. Abbafati, Z. Abebe, M. Afarideh, A. Aggarwal, S. Agrawal, T. Akinyemiju, F. Alahdab, U. Bacha, V. F. Bachman, H. Badali, A. Badawi, I. M. Bensenor, E. Bernabe, S. K. K. Biadgilign, S. H. Biryukov, L. E. Cahill, J. J. Carrero, K. M. Cercy, L. Dandona, R. Dandona, A. K. Dang, M. G. Degefa, M. El Sayed Zaki, A. Esteghamati, S. Esteghamati, J. Fanzo, C. S. e. S. Farinha, M. S. Farvid, F. Farzadfar, V. L. Feigin, J. C. Fernandes, L. S. Flor, N. A. Foigt, M. H. Forouzanfar, M. Ganji, J. M. Geleijnse, R. F. Gillum, A. C. Goulart, G. Grosso, I. Guessous, S. Hamidi, G. J. Hankey, S. Harikrishnan, H. Y. Hassen, S. I. Hay, C. L. Hoang, M. Horino, F. Islami, M. D. Jackson, S. L. James, L. Johansson, J. B. Jonas, A. Kasaeian, Y. S. Khader, I. A. Khalil, Y. H. Khang, R. W. Kimokoti, Y. Kokubo, G. A. Kumar, T. Lallukka, A. D. Lopez, S. Lorkowski, P. A. Lotufo, R. Lozano, R. Malekzadeh, W. März, T. Meier, Y. A. Melaku, W. Mendoza, G. B. M. Mensink, R. Micha, T. R. 
Miller, M. Mirarefin, V. Mohan, A. H. Mokdad, D. Mozaffarian, G. Nagel, M. Naghavi, C. T. Nguyen, M. R. Nixon, K. L. Ong, D. M. Pereira, H. Poustchi, M. Qorbani, R. K. Rai, C. Razo-García, C. D. Rehm, J. A. Rivera, S. Rodríguez-Ramírez, G. Roshandel, G. A. Roth, J. Sanabria, T. G. Sánchez-Pimienta, B. Sartorius, J. Schmidhuber, A. E. Schutte, S. G. Sepanlou, M. J. Shin, R. J. D. Sorensen, M. Springmann, L. Szponar, A. L. Thorne-Lyman, A. G. Thrift, M. Touvier, B. X. Tran, S. Tyrovolas, K. N. Ukwaja, I. Ullah, O. A. Uthman, M. Vaezghasemi, T. J. Vasankari, S. E. Vollset, T. Vos, G. T. Vu, L. G. Vu, E. Weiderpass, A. Werdecker, T. Wijeratne, W. C. Willett, J. H. Wu, G. Xu, N. Yonemoto, C. Yu and C. J. L. Murray, Health effects of dietary risks in 195 countries, 1990-2017: a systematic analysis for the Global Burden of Disease Study 2017, Lancet, 2019, 393, 1958-1972. D. Mozaffarian, Dietary and Policy Priorities for Cardiovascular Disease, Diabetes, and Obesity, Circulation, 2016, 133, 187-225. R. H. Liu, Health-promoting components of fruits and vegetables in the diet, $A d v$. Nutr., 2013, 4, 384S-92S. M. G. L. Hertog, E. J. M. Feskens, D. Kromhout, P. C. H. Hollman and M. B. Katan, Dietary antioxidant flavonoids and risk of coronary heart disease: the Zutphen Elderly Study, Lancet, 1993, 342, 1007-1011. I. C. W. Arts and P. C. H. Hollman, Polyphenols and disease risk in epidemiologic studies., Am. J. Clin. Nutr., 2005, 82, 317S-325S.

B. A. Graf, P. E. Milbury and J. B. Blumberg, Flavonols, flavones, flavanones, and human health: Epidemiological evidence, J. Med. Food, 2005, 8, 281-290.

8 J. J. Peterson, J. T. Dwyer, P. F. Jacques and M. L. McCullough, Associations between flavonoids and cardiovascular disease incidence or mortality in European and US 
S. Kumar and A. K. Pandey, Chemistry and biological activities of flavonoids: An overview, Sci. World J., 2013, DOI: 10.1155/2013/162750. biological significance?, Crit. Rev. Food Sci. Nutr., 2017, 57, 1874-1905. HS Tuli, Current Aspects of Flavonoids: Their Role in Cancer Treatment, Springer, Singapore, 2019.

D. O. Kennedy and E. L. Wightman, Herbal extracts and phytochemicals: Plant secondary metabolites and the enhancement of human brain function, Adv. Nutr., 2011, 2, 32-50.

13 J. J. Peterson, J. T. Dwyer, P. F. Jacques and M. L. McCullough, Improving the estimation of flavonoid intake for study of health outcomes, Nutr. Rev., 2015, 73, 553576.

G. Williamson, C. D. Kay and A. Crozier, The bioavailability, transport, and bioactivity of dietary flavonoids: A review from a historical perspective, Compr. Rev. Food Sci. Food Saf., 2018, 17, 1054-1112.

D. Del Rio, A. Rodriguez-Mateos, J. P. E. Spencer, M. Tognolini, G. Borges and A. evidence of protective effects against chronic diseases, Antioxidants Redox Signal., 2013, 18, 1818-1892.

J. M. Holden, S. A. Bhagwat, D. B. Haytowitz, S. E. Gebhardt, J. T. Dwyer, J. Peterson, G. R. Beecher, A. L. Eldridge and D. Balentine, Development of a database of critically evaluated flavonoids data: Application of USDA's data quality evaluation system, J. Food Compos. Anal., 2005, 18, 829-844. 
17 J. Pérez-Jiménez, V. Neveu, F. Vos and A. Scalbert, Systematic analysis of the content of 502 Polyphenols in 452 foods and beverages: An application of the phenol-explorer database, J. Agric. Food Chem., 2010, 58, 4959-4969.

18 P. C. H. Hollman, M. G. L. Hertog and M. B. Katan, Analysis and health effects of flavonoids, Food Chem., 1996, 57, 43-46.

19 V. Neveu, J. Perez-Jiménez, F. Vos, V. Crespy, L. du Chaffaut, L. Mennen, C. Knox, R. Eisner, J. Cruz, D. Wishart and A. Scalbert, Phenol-Explorer: an online comprehensive database on polyphenol contents in foods, Database, 2010, DOI: 10.1093/database/bap024.

D. Haytowitz, X. Wu and S. Bhagwat, USDA Database for the Flavonoid Content of Selected Foods, Release 3.3, U.S. Department of Agriculture, Agricultural Research Service, Nutrient Data Laboratory, 2018.

21 J. Pérez-Jiménez, L. Fezeu, M. Touvier, N. Arnault, C. Manach, S. Hercberg, P. Galan and A. Scalbert, Dietary intake of 337 polyphenols in French adults, Am. J. Clin. Nutr., $2011,93,1220-1228$.

F. Perez-Vizcaino and J. Duarte, Flavonols and cardiovascular disease, Mol. Aspects Med., 2010, 31, 478-494.

S. Bhagwat, D. B. Haytowitz and J. M. Holden, Database for the Isoflavone Content of Selected Foods, Release 2.0, U.S. Department of Agriculture, Agricultural Research Service, Nutrient Data Laboratory, 2008.

24 E. Capanoglu, D. Boyacioglu, R. C. H. De Vos, R. D. Hall and J. Beekwilder, Procyanidins in fruit from Sour cherry (Prunus cerasus) differ strongly in chainlength from those in Laurel cherry (Prunus lauracerasus) and Cornelian cherry (Cornus mas), J. Berry Res., 2011, 1, 137-146. 
R. M. Hackman, J. A. Polagruto, Q. Y. Zhu, B. Sun, H. Fujii and C. L. Keen, Flavanols: Digestion, absorption and bioactivity, Phytochem. Rev., 2008, 7, 195-208.

S. Sang, J. D. Lambert, C. T. Ho and C. S. Yang, The chemistry and biotransformation of tea constituents, Pharmacol. Res., 2011, 64, 87-99.

S. Bhagwat, D. B. Haytowitz, R. L. Prior, L. Gu, J. Hammerstone, S. E. Gebhardt, M. Kelm, D. Cunningham, G. R. Beecher and J. M. Holden, USDA database for proanthocyanidin content of selected foods, U.S. Department of Agriculture, Agricultural Research Service, 2004.

R. Escobar-Cévoli, C. Castro-Espín, V. Béraud, G. Buckland and R. Zamora-Ros, in Flavonoids - From Biosynthesis to Human Health, ed. G. Justino, IntechOpen, Croatia, 2017.

C. Braicu, M. R. Ladomery, V. S. Chedea, A. Irimie and I. Berindan-Neagoe, The relationship between the structure and biological actions of green tea catechins, Food Chem., 2013, 141, 3282-3289.

R. Zamora-Ros, V. Knaze, J. A. Rothwell, B. Hémon, A. Moskal, K. Overvad, A. Tjønneland, C. Kyrø, G. Fagherazzi, M. C. Boutron-Ruault, M. Touillaud, V. Katzke, T. Kühn, H. Boeing, J. Förster, A. Trichopoulou, E. Valanou, E. Peppa, D. Palli, C. Agnoli, F. Ricceri, R. Tumino, M. S. de Magistris, P. H. M. Peeters, H. Bas BuenoDe-Mesquita, D. Engeset, G. Skeie, A. Hjartåker, V. Menéndez, A. Agudo, E. MolinaMontes, J. M. Huerta, A. Barricarte, P. Amiano, E. Sonestedt, L. M. Nilsson, R. Landberg, T. J. Key, K. T. Khaw, N. J. Wareham, Y. Lu, N. Slimani, I. Romieu, E. Riboli and A. Scalbert, Dietary polyphenol intake in europe: The european prospective investigation into cancer and nutrition (EPIC) study, Eur. J. Nutr., 2016, 55, 1359 1375. 
31 J. Kühnau, Flavonoids. A class of semi-essential food components: their role in human nutrition., World Rev. Nutr. Diet., 1976, 24, 117-191.

32 K. Herrmann, Flavonols and flavones in food plants: a review, Int. J. Food Sci. Technol., 1976, 11, 433-448.

33 M. G. L. Hertog, P. C. H. Hollman and M. B. Katan, Content of potentially anticarcinogenic flavonoids of 28 vegetables and 9 fruits commonly consumed in the Netherlands, J. Agric. Food Chem., 1992, 40, 2379-2383.

34 M. G. L. Hertog, P. C. H. Hollman and B. van de Putte, Content of potentially anticarcinogenic flavonoids of tea infusions, wines, and fruit juices, J. Agric. Food Chem., 1993, 41, 1242-1246.

T. Hirvonen, J. Virtamo, P. Korhonen, D. Albanes and P. Pietinen, Intake of flavonoids, carotenoids, vitamins C and E, and risk of stroke in male smokers, Stroke, 2000, 31, 2301-2306.

P. Knekt, R. Järvinen, A. Reunanen and J. Maatela, Flavonoid intake and coronary mortality in Finland: A cohort study, Br. Med. J., 1996, 312, 478-481.

37 E. B. Rimm, M. B. Katan, A. Ascherio, M. J. Stampfer and W. C. Willett, Relation between intake of flavonoids and risk for coronary heart disease in male health professionals, Ann. Intern. Med., 1996, 125, 384-389.

38 M. G. L. Hertog, P. M. Sweetnam, A. M. Fehily, P. C. Elwood and D. Kromhout, Antioxidant flavonols and ischemic heart disease in a Welsh population of men: The Caerphilly Study, Am. J. Clin. Nutr., 1997, 65, 1489-1494.

39 L. Yochum, L. H. Kushi, K. Meyer and A. R. Folsom, Dietary flavonoid intake and risk of cardiovascular disease in postmenopausal women, Am. J. Epidemiol., 1999, 149, 943-949. 
T. Hirvonen, P. Pietinen, M. Virtanen, M. L. Ovaskainen, S. Häkkinen, D. Albanes and J. Virtamo, Intake of flavonols and flavones and risk of coronary heart disease in male smokers, Epidemiology, 2001, 12, 62-67.

J. M. Geleijnse, L. J. Launer, D. A. M. Van Der Kuip, A. Hofman and J. C. M. Witteman, Inverse association of tea and flavonoid intakes with incident myocardial infarction: The Rotterdam Study, Am. J. Clin. Nutr., 2002, 75, 880-886. H. D. Sesso, J. M. Gaziano, S. Liu and J. E. Buring, Flavonoid intake and the risk of cardiovascular disease in women, Am. J. Clin. Nutr., 2003, 77, 1400-1408. I. C. W. Arts, B. Van De Putte and P. C. H. Hollman, Catechin contents of foods commonly consumed in The Netherlands. 1. Fruits, vegetables, staple foods, and processed foods, J. Agric. Food Chem., 2000, 48, 1746-1751.

I. C. W. Arts, P. C. H. Hollman, E. J. M. Feskens, H. B. Bueno de Mesquita and D. Kromhout, Catechin intake might explain the inverse relation between tea consumption and ischemic heart disease: The Zutphen Elderly Study, Am. J. Clin. Nutr., 2001, 74, 227-232.

6 I. C. W. Arts, D. R. Jacobs, L. J. Harnack, M. Gross and A. R. Folsom, Dietary catechins in relation to coronary heart disease death among postmenopausal women, Epidemiology, 2001, 12, 668-675.

P. Knekt, J. Kumpulainen, R. Järvinen, H. Rissanen, M. Heliövaara, A. Reunanen, T. Hakulinen and A. Aromaa, Flavonoid intake and risk of chronic diseases, Am. J. Clin. Nutr., 2002, 76, 560-568. 
48 S. Hendrich and P. A. Murphy, in Handbook of Nutraceuticals and Functional Foods, CRC Press, Boca Roton, 2nd ed., 2016.

49 S. A. Bhagwat, D. B. Haytowitz, S. I. Wasswa-Kintu and P. R. Pehrsson, Process of formulating USDA's expanded flavonoid database for the assessment of dietary intakes: A new tool for epidemiological research, Br. J. Nutr., 2015, 114, 472-480.

50 P. J. Mink, C. G. Scrafford, L. M. Barraj, L. Harnack, C. P. Hong, J. A. Nettleton and D. R. Jacobs, Flavonoid intake and cardiovascular disease mortality: A prospective study in postmenopausal women, Am. J. Clin. Nutr., 2007, 85, 895-909.

51 N. P. Bondonno, J. R. Lewis, L. C. Blekkenhorst, C. P. Bondonno, J. H. Shin, K. D. Croft, R. J. Woodman, G. Wong, W. H. Lim, B. Gopinath, V. M. Flood, J. Russell, P. Mitchell and J. M. Hodgson, Association of flavonoids and flavonoid-rich foods with all-cause mortality: The Blue Mountains Eye Study, Clin. Nutr., 2019, 39, 141-150.

K. L. Ivey, M. K. Jensen, J. M. Hodgson, A. H. Eliassen, A. Cassidy and E. B. Rimm, Association of flavonoid-rich foods and flavonoids with risk of all-cause mortality, $B r$. J. Nutr., 2017, 117, 1470-1477.

P. F. Jacques, A. Cassidy, G. Rogers, J. J. Peterson and J. T. Dwyer, Dietary flavonoid intakes and CVD incidence in the Framingham Offspring Cohort, Br. J. Nutr., 2015, 114, 1496-1503.

54 M. L. McCullough, J. J. Peterson, R. Patel, P. F. Jacques, R. Shah and J. T. Dwyer, Flavonoid intake and cardiovascular disease mortality in a prospective cohort of US adults, Am. J. Clin. Nutr., 2012, 95, 454-464.

55 J. Mursu, S. Voutilainen, T. Nurmi, T. P. Tuomainen, S. Kurl and J. T. Salonen, Flavonoid intake and the risk of ischaemic stroke and CVD mortality in middle-aged Finnish men: The Kuopio Ischaemic Heart Disease Risk Factor Study, Br. J. Nutr., 
A. Cassidy, K. J. Mukamal, L. Liu, M. Franz, A. H. Eliassen and E. B. Rimm, High anthocyanin intake is associated with a reduced risk of myocardial infarction in young and middle-aged women, Circulation, 2013, 127, 188-196. M. E. Goetz, S. E. Judd, M. M. Safford, T. J. Hartman, W. M. McClellan and V. Vaccarino, Dietary flavonoid intake and incident coronary heart disease: The REasons for Geographic and Racial Differences in Stroke (REGARDS) study, Am. J. Clin. Nutr., 2016, 104, 1236-1244.

8 J. A. Rothwell, M. Urpi-Sarda, M. Boto-Ordoñez, C. Knox, R. Llorach, R. Eisner, J. Cruz, V. Neveu, D. Wishart, C. Manach, C. Andres-Lacueva and A. Scalbert, PhenolExplorer 2.0: a major update of the Phenol-Explorer database integrating data on polyphenol metabolism and pharmacokinetics in humans and experimental animals., Database, 2012, DOI: 10.1093/database/bas031.

59 J. A. Rothwell, J. Perez-Jimenez, V. Neveu, A. Medina-Remón, N. M’Hiri, P. GarcíaLobato, C. Manach, C. Knox, R. Eisner, D. S. Wishart and A. Scalbert, PhenolExplorer 3.0: A major update of the Phenol-Explorer database to incorporate data on the effects of food processing on polyphenol content, Database, 2013, DOI: 10.1093/database/bat070.

S. Adriouch, A. Lampuré, A. Nechba, J. Baudry, K. Assmann, E. Kesse-Guyot, S. Hercberg, A. Scalbert, M. Touvier and L. K. Fezeu, Prospective association between total and specific dietary polyphenol intakes and cardiovascular disease risk in the Nutrinet-Santé French Cohort, Nutrients, 2018, 10, 1587.

61 N. P. Bondonno, F. Dalgaard, C. Kyrø, K. Murray, C. P. Bondonno, J. R. Lewis, K. D. Croft, G. Gislason, A. Scalbert, A. Cassidy, A. Tjønneland, K. Overvad and J. M. 
Hodgson, Flavonoid intake is associated with lower mortality in the Danish Diet Cancer and Health Cohort, Nat. Commun., 2019, 10, DOI: 10.1038/s41467-01911622-x.

62 F. Dalgaard, N. P. Bondonno, K. Murray, C. P. Bondonno, J. R. Lewis, K. D. Croft, C. Kyrø, G. Gislason, A. Scalbert, A. Cassidy, A. Tjønneland, K. Overvad and J. M. Hodgson, Associations between habitual flavonoid intake and hospital admissions for atherosclerotic cardiovascular disease: a prospective cohort study, Lancet Planet. Heal., 2019, 3, e450-e459.

63 K. L. Ivey, J. R. Lewis, R. L. Prince and J. M. Hodgson, Tea and non-tea flavonol intakes in relation to atherosclerotic vascular disease mortality in older women, $B r . J$. Nutr., 2013, 110, 1648-1655.

64 A. Tresserra-Rimbau, E. B. Rimm, A. Medina-Remón, M. A. Martínez-González, R. de la Torre, D. Corella, J. Salas-Salvadó, E. Gómez-Gracia, J. Lapetra, F. Arós, M. Fiol, E. Ros, L. Serra-Majem, X. Pintó, G. T. Saez, J. Basora, J. V. Sorlí, J. A. Martínez, E. Vinyoles, V. Ruiz-Gutiérrez, R. Estruch and R. M. Lamuela-Raventós, Inverse association between habitual polyphenol intake and incidence of cardiovascular events in the PREDIMED study, Nutr. Metab. Cardiovasc. Dis., 2014, 24, 639-647.

65 V. Knaze, J. A. Rothwell, R. Zamora-Ros, A. Moskal, C. Kyrø, P. Jakszyn, G. Skeie, E. Weiderpass, M. S. De Magistris, C. Agnoli, S. Westenbrink, E. Sonestedt, A. Trichopoulou, E. Vasilopoulou, E. Peppa, E. Ardanaz, J. M. Huerta, H. Boeing, F. R. Mancini, A. Scalbert and N. Slimani, A new food-composition database for 437 polyphenols in 19,899 raw and prepared foods used to estimate polyphenol intakes in adults from 10 European countries, Am. J. Clin. Nutr., 2018, 108, 517-524. 
66 J. Gry, L. Black, F. D. Eriksen, K. Pilegaard, J. Plumb, M. Rhodes, D. Sheehan, M. Kiely and P. A. Kroon, EuroFIR-BASIS - a combined composition and biological activity database for bioactive compounds in plant-based foods, Trends Food Sci. Technol., 2007, 18, 434-444.

67 V. Ponzo, I. Goitre, M. Fadda, R. Gambino, A. De Francesco, L. Soldati, L. Gentile, P. Magistroni, M. Cassader and S. Bo, Dietary flavonoid intake and cardiovascular risk: A population-based cohort study, J. Transl. Med., 2015, 13, 218.

68 R. Zamora-Ros, M. Touillaud, J. A. Rothwell, I. Romieu and A. Scalbert, Measuring exposure to the polyphenol metabolome in observational epidemiologic studies: Current tools and applications and their limits, Am. J. Clin. Nutr., 2014, 100, 11-26.

69 K. L. Ivey, K. Croft, R. L. Prince and J. M. Hodgson, Comparison of flavonoid intake assessment methods, Food Funct., 2016, 7, 3748-3759.

70 A. Satija, E. Yu, W. C. Willett and F. B. Hu, Understanding nutritional epidemiology and its role in policy, Adv. Nutr., 2015, 6, 5-18.

71 F. E. Thompson and A. F. Subar, in Nutrition in the Prevention and Treatment of Disease, Academic Press, 4th ed., 2017.

72 L. S. Freedman, A. Schatzkin, D. Midthune and V. Kipnis, Dealing with dietary measurement error in nutritional cohort studies, J. Natl. Cancer Inst., 2011, 103, 10861092.

73 S. Somerset and K. Papier, A food frequency questionnaire validated for estimating dietary flavonoid intake in an Australian population, Nutr. Cancer, 2014, 66, 12001210.

74 K. Kent and K. E. Charlton, Development, validation and reproducibility of a food frequency questionnaire to measure flavonoid intake in older Australian adults, Nutr. 
M. E. Rollo, R. L. Williams, T. Burrows, S. I. Kirkpatrick, T. Bucher and C. E. Collins, What are they really eating? A review on new approaches to dietary intake assessment and validation, Curr. Nutr. Rep., 2016, 5, 307-314.

R. Zamora-Ros, C. Jiménez, R. Cleries, A. Agudo, M. J. Sánchez, E. SánchezCantalejo, E. Molina-Montes, C. Navarro, M. D. Chirlaque, J. María Huerta, P. Amiano, M. L. Redondo, A. Barricarte and C. A. González, Dietary flavonoid and lignan intake and mortality in a Spanish cohort, Epidemiology, 2013, 24, 726-733.

79 A. B. Santhakumar, A. C. Bulmer and I. Singh, A review of the mechanisms and effectiveness of dietary polyphenols in reducing oxidative stress and thrombotic risk, J. Hum. Nutr. Diet., 2014, 27, 1-21.

D. Maaliki, A. A. Shaito, G. Pintus, A. El-Yazbi and A. H. Eid, Flavonoids in hypertension: a brief review of the underlying mechanisms, Curr. Opin. Pharmacol., $2019,45,57-65$. flavonoids and preventive effects against cardiovascular disease, J. Cardiovasc. Pharmacol., 2019, 75, 1-9.

M. Lilamand, E. Kelaiditi, S. Guyonnet, R. Antonelli Incalzi, A. Raynaud-Simon, B. Vellas and M. Cesari, Flavonoids and arterial stiffness: Promising perspectives, Nutr. 
R. Zamora-Ros, V. Knaze, L. Luján-Barroso, I. Romieu, A. Scalbert, N. Slimani, A. Hjartåker, D. Engeset, G. Skeie, K. Overvad, L. Bredsdorff, A. Tjonneland, J. Halkjær, T. J. Key, K. T. Khaw, A. A. Mulligan, A. Winkvist, I. Johansson, H. Bas Bueno-DeMesquita, P. H. M. Peeters, P. Wallström, U. Ericson, V. Pala, M. S. De Magistris, S. Polidoro, R. Tumino, A. Trichopoulou, V. Dilis, M. Katsoulis, J. María Huerta, V. Martínez, M. J. Sánchez, E. Ardanaz, P. Amiano, B. Teucher, V. Grote, B. Bendinelli, H. Boeing, J. Förster, M. Touillaud, F. Perquier, G. Fagherazzi, V. Gallo, E. Riboli and C. A. González, Differences in dietary intakes, food sources and determinants of total flavonoids between Mediterranean and non-Mediterranean countries participating in the European Prospective Investigation into Cancer and Nutrition (EPIC) study, Br. J. Nutr., 2013, 109, 1498-1507.

O. K. Chun, S. J. Chung and W. O. Song, Estimated dietary flavonoid intake and major food sources of U.S. adults, J. Nutr., 2007, 137, 1244-1252. D. Levy, Impact of high-normal blood pressure on the risk of cardiovascular disease, N. Engl. J. Med., 2001, 345, 1291-1297. A. Cassidy, Dietary intakes of flavan-3-ols and cardiometabolic health: Systematic review and meta-analysis of randomized trials and prospective cohort studies, Am. J. Clin. Nutr., 2019, 110, 1067-1078.

7 P. O. Bonetti, L. O. Lerman and A. Lerman, Endothelial dysfunction: A marker of atherosclerotic risk, Arterioscler. Thromb. Vasc. Biol., 2003, 23, 168-175.

E. Falk, Pathogenesis of atherosclerosis, J. Am. Coll. Cardiol., 2006, 47, 7-12. 
89 C. P. Bondonno, K. D. Croft, N. Ward, M. J. Considine and J. M. Hodgson, Dietary flavonoids and nitrate: Effects on nitric oxide and vascular function, Nutr. Rev., 2015, 73, 216-235.

90 K. D. Croft, D. Zhang, R. Jiang, A. Ayer, S. Shengule, R. J. Payne, N. C. Ward and R. Stocker, Structural requirements of flavonoids to induce heme oxygenase-1 expression, Free Radic. Biol. Med., 2017, 113, 165-175.

91 L. Guerrero, J. Castillo, M. Quiñones, S. Garcia-Vallvé, L. Arola, G. Pujadas and B. Muguerza, Inhibition of angiotensin-converting enzyme activity by flavonoids: Structure-activity relationship studies, PLoS One, 2012, 7, DOI: 10.1371/journal.pone.0049493.

92 A. García-Lafuente, E. Guillamón, A. Villares, M. A. Rostagno and J. A. Martínez, Flavonoids as anti-inflammatory agents: Implications in cancer and cardiovascular disease, Inflamm. Res., 2009, 58, 537-552.

93 L. P. Morera, G. N. Marchiori, L. A. Medrano and M. D. Defagó, Stress, dietary patterns and cardiovascular disease: A mini-review, Front. Neurosci., 2019, 13, DOI: doi.org/10.3389/fnins.2019.01226.

94 G. A. Roth, C. Johnson, A. Abajobir, F. Abd-Allah, S. F. Abera, G. Abyu, M. Ahmed, B. Aksut, T. Alam, K. Alam, F. Alla, N. Alvis-Guzman, S. Amrock, H. Ansari, J. Ärnlöv, H. Asayesh, T. M. Atey, L. Avila-Burgos, A. Awasthi, A. Banerjee, A. Barac, T. Bärnighausen, L. Barregard, N. Bedi, E. Belay Ketema, D. Bennett, G. Berhe, Z. Bhutta, S. Bitew, J. Carapetis, J. J. Carrero, D. C. Malta, C. A. Castañeda-Orjuela, J. Castillo-Rivas, F. Catalá-López, J. Y. Choi, H. Christensen, M. Cirillo, L. Cooper, M. Criqui, D. Cundiff, A. Damasceno, L. Dandona, R. Dandona, K. Davletov, S. Dharmaratne, P. Dorairaj, M. Dubey, R. Ehrenkranz, M. El Sayed Zaki, E. J. A. 
Faraon, A. Esteghamati, T. Farid, M. Farvid, V. Feigin, E. L. Ding, G. Fowkes, T. Gebrehiwot, R. Gillum, A. Gold, P. Gona, R. Gupta, T. D. Habtewold, N. HafeziNejad, T. Hailu, G. B. Hailu, G. Hankey, H. Y. Hassen, K. H. Abate, R. Havmoeller, S. I. Hay, M. Horino, P. J. Hotez, K. Jacobsen, S. James, M. Javanbakht, P. Jeemon, D. John, J. Jonas, Y. Kalkonde, C. Karimkhani, A. Kasaeian, Y. Khader, A. Khan, Y. H. Khang, S. Khera, A. T. Khoja, J. Khubchandani, D. Kim, D. Kolte, S. Kosen, K. J. Krohn, G. A. Kumar, G. F. Kwan, D. K. Lal, A. Larsson, S. Linn, A. Lopez, P. A. Lotufo, H. M. A. El Razek, R. Malekzadeh, M. Mazidi, T. Meier, K. G. Meles, G. Mensah, A. Meretoja, H. Mezgebe, T. Miller, E. Mirrakhimov, S. Mohammed, A. E. Moran, K. I. Musa, J. Narula, B. Neal, F. Ngalesoni, G. Nguyen, C. M. Obermeyer, M. Owolabi, G. Patton, J. Pedro, D. Qato, M. Qorbani, K. Rahimi, R. K. Rai, S. Rawaf, A. Ribeiro, S. Safiri, J. A. Salomon, I. Santos, M. Santric Milicevic, B. Sartorius, A. Schutte, S. Sepanlou, M. A. Shaikh, M. J. Shin, M. Shishehbor, H. Shore, D. A. S. Silva, E. Sobngwi, S. Stranges, S. Swaminathan, R. Tabarés-Seisdedos, N. Tadele Atnafu, F. Tesfay, J. S. Thakur, A. Thrift, R. Topor-Madry, T. Truelsen, S. Tyrovolas, K. N. Ukwaja, O. Uthman, T. Vasankari, V. Vlassov, S. E. Vollset, T. Wakayo, D. Watkins, R. Weintraub, A. Werdecker, R. Westerman, C. S. Wiysonge, C. Wolfe, A. Workicho, G. Xu, Y. Yano, P. Yip, N. Yonemoto, M. Younis, C. Yu, T. Vos, M. Naghavi and C. Murray, Global, regional, and national burden of cardiovascular diseases for 10 causes, 1990 to 2015, J. Am. Coll. Cardiol., 2017, 70, 1-25.

95 J. Stewart, G. Manmathan and P. Wilkinson, Primary prevention of cardiovascular disease: A review of contemporary guidance and literature, JRSM Cardiovasc. Dis., $2017,6,1-9$. 
W. Herrington, B. Lacey, P. Sherliker, J. Armitage and S. Lewington, Epidemiology of atherosclerosis and the potential to reduce the global burden of atherothrombotic disease, Circ. Res., 2016, 118, 535-546.

F. Peter Libby, MD, Robert O. Bonow, MD, Douglas P. Zipes, MD and Douglas L. Mann, MD, Braunwald's Heart Disease: A Textbook of Cardiovascular Medicine, Saunders Elsevier, Philadelphia, 8th ed., 2007.

D. Vauzour, A. Rodriguez-Mateos, G. Corona, M. J. Oruna-Concha and J. P. E. action, Nutrients, 2010, 2, 1106-1131.

100 C. G. Fraga, K. D. Croft, D. O. Kennedy and F. A. Tomás-Barberán, The effects of polyphenols and other bioactives on human health, Food Funct., 2019, 10, 514-528.

101 Y. Kokubo, H. Iso, J. Ishihara, K. Okada, M. Inoue and S. Tsugane, Association of dietary intake of soy, beans, and isoflavones with risk of cerebral and myocardial infarctions in Japanese populations: The Japan Public Health Center-Based (JPHC) Study Cohort I, Circulation, 2007, 116, 2553-2562.

102 Y. T. Van Der Schouw, S. Kreijkamp-Kaspers, P. H. M. Peeters, L. Keinan-Boker, E. B. Rimm and D. E. Grobbee, Prospective study on usual dietary phytoestrogen intake and cardiovascular disease risk in Western women, Circulation, 2005, 111, 465-471.

3 A. Vogiatzoglou, A. A. Mulligan, A. Bhaniani, M. A. H. Lentjes, A. McTaggart, R. N. Luben, C. Heiss, M. Kelm, M. W. Merx, J. P. E. Spencer, H. Schroeter, K. T. Khaw and G. G. C. Kuhnle, Associations between flavan-3-ol intake and CVD risk in the Norfolk cohort of the European Prospective Investigation into Cancer (EPIC-Norfolk), Free Radic. Biol. Med., 2015, 84, 1-10. 
Lopes, A. Gea, M. A. Martinez-Gonzalez and M. Bes-Rastrollo, Total polyphenol intake, polyphenol subtypes and incidence of cardiovascular disease: The SUN cohort study, Nutr. Metab. Cardiovasc. Dis., 2019, 29, 69-78.

105 G. Grosso, A. Micek, J. Godos, A. Pajak, S. Sciacca, F. Galvano and E. L. Giovannucci, Dietary flavonoid and lignan intake and mortality in prospective cohort studies: systematic review and dose-response meta-analysis, Am. J. Epidemiol., 2017, $185,1304-1316$

106 X. M. Liu, Y. J. Liu, Y. Huang, H. J. Yu, S. Yuan, B. W. Tang, P. G. Wang and Q. Q. He, Dietary total flavonoids intake and risk of mortality from all causes and cardiovascular disease in the general population: A systematic review and metaanalysis of cohort studies, Mol. Nutr. Food Res., 2017, 61, DOI: 10.1002/mnfr.201601003.

107 Y. Kim and Y. Je, Flavonoid intake and mortality from cardiovascular disease and all causes: A meta-analysis of prospective cohort studies, Clin. Nutr. ESPEN, 2017, 20, $68-77$.

108 X. Wang, Y. Y. Ouyang, J. Liu and G. Zhao, Flavonoid intake and risk of CVD: A systematic review and meta-analysis of prospective cohort studies, Br. J. Nutr., 2014, $111,1-11$.

109 R. Kimble, K. M. Keane, J. K. Lodge and G. Howatson, Dietary intake of anthocyanins and risk of cardiovascular disease: A systematic review and metaanalysis of prospective cohort studies, Crit. Rev. Food Sci. Nutr., 2019, 59, 1-12.

110 N. Potischman and D. L. Weed, Causal criteria in nutritional epidemiology, Am. J. Clin. Nutr., 1999, 69, 1309S-1314S.

111 J. I. Dower, J. M. Geleijnse, P. C. H. Hollman, S. S. Soedamah-Muthu and D. 
Kromhout, Dietary epicatechin intake and 25-y risk of cardiovascular mortality: The Zutphen Elderly Study, Am. J. Clin. Nutr., 2016, 104, 58-64.

112 M. Talaei, W. P. Koh, R. M. van Dam, J. M. Yuan and A. Pan, Dietary soy intake is not associated with risk of cardiovascular disease mortality in Singapore Chinese adults, J. Nutr., 2014, 144, 921-928.

113 J. H. Hankin, D. O. Stram, K. Arakawa, S. Park, S. H. Low, H. P. Lee and M. C. Yu, Singapore Chinese Health Study: Development, validation, and calibration of the quantitative food frequency questionnaire, Nutr. Cancer, 2001, 39, 187-195.

114 M. Kimira, Y. Arai, K. Shimoi and S. Watanabe, Japanese intake of flavonoids and isoflavonoids from foods, J. Epidemiol., 1998, 8, 168-175.

115 C. Steenbergen and N. G. Frangogiannis, in Muscle - Fundamental Biology and Mechanisms of Disease, Academic Press Elsevier, 2012.

116 J. Müller-Nordhorn and S. N. Willich, in International Encyclopedia of Public Health, 2016.

117 M. G. L. Hertog, D. Kromhout, C. Aravanis, H. Blackburn, R. Buzina, F. Fidanza, S. Giampaoli, A. Jansen, A. Menotti, S. Nedeljkovic, M. Pekkarinen, B. S. Simic, H. Toshima, E. J. M. Feskens, P. C. H. Hollman and M. B. Katan, Flavonoid intake and long-term risk of coronary heart disease and cancer in the Seven Countries Study, Arch. Intern. Med., 1995, 155, 381-386.

118 M. G. L. Hertog, E. J. M. Feskens and D. Kromhout, Antioxidant flavonols and coronary heart disease risk, Lancet, 1997, 349, 699.

119 A. Cassidy, M. Bertoia, S. Chiuve, A. Flint, J. Forman and E. B. Rimm, Habitual intake of anthocyanins and flavanones and risk of cardiovascular disease in men, $\mathrm{Am}$. J. Clin. Nutr., 2016, 104, 587-594. 
120 W. Jiang, H. Wei and B. He, Dietary flavonoids intake and the risk of coronary heart disease: A dose-response meta-analysis of 15 prospective studies, Thromb. Res., 2015, $135,459-463$.

121 R. R. Huxley and H. A. W. Neil, The relation between dietary flavonol intake and coronary heart disease mortality: A meta-analysis of prospective cohort studies, Eur. J. Clin. Nutr., 2003, 57, 904-908.

122 J. Marniemi, E. Alanen, O. Impivaara, R. Seppänen, P. Hakala, T. Rajala and T. Rönnemaa, Dietary and serum vitamins and minerals as predictors of myocardial infarction and stroke in elderly subjects, Nutr. Metab. Cardiovasc. Dis., 2005, 15, 188197.

123 X. Tong, Q. Yang, M. D. Ritchey, M. G. George, S. L. Jackson, C. Gillespie and R. K. Merritt, The burden of cerebrovascular disease in the United States, Prev. Chronic Dis., 2019, 16, DOI: 10.5888/pcd16.180411

124 N. McCormick, V. Bhole, D. Lacaille and J. A. Avina-Zubieta, Validity of diagnostic codes for acute stroke in administrative databases: A systematic review, PLoS One, 2015, 10, DOI: 10.1371/journal.pone.0135834

125 A. Chandra, C. R. Stone, X. Du, W. A. Li, M. Huber, R. Bremer, X. Geng and Y. Ding, The cerebral circulation and cerebrovascular disease II: Pathogenesis of cerebrovascular disease, Brain Circ., 2019, 3, 57-65.

126 J. C. Grotta, G. W. Albers, J. P. Broderick, S. E. Kasner, E. H. Lo, A. D. Mendelow, R. L. Sacco and L. K. S. Wong, Stroke: Pathophysiology, Diagnosis, and Management, Elsevier, 6th ed., 2015.

127 R. L. Sacco, S. E. Kasner, J. P. Broderick, L. R. Caplan, J. J. (Buddy) Connors, A. Culebras, M. S. V. Elkind, M. G. George, A. D. Hamdan, R. T. Higashida, B. L. Hoh, 
L. S. Janis, C. S. Kase, D. O. Kleindorfer, J.-M. Lee, M. E. Moseley, E. D. Peterson, T. N. Turan, A. L. Valderrama and H. V. Vinters, An updated definition of stroke for the 21st century, Stroke, 2013, 44, 2064-2089.

128 A. Rodriguez-Mateos, D. Vauzour, C. G. Krueger, D. Shanmuganayagam, J. Reed, L. Calani, P. Mena, D. Del Rio and A. Crozier, Bioavailability, bioactivity and impact on health of dietary flavonoids and related compounds: an update, Arch. Toxicol., 2014, 88, 1803-1853.

129 M. E. Goetz, S. E. Judd, T. J. Hartman, W. McClellan, A. Anderson and V. Vaccarino, Flavanone intake is inversely associated with risk of incident ischemic stroke in the REasons for Geographic and Racial Differences in Stroke (REGARDS) Study, $J$. Nutr., 2016, 146, 2233-2243.

130 Z. Vokó, M. Hollander, A. Hofman, P. J. Koudstaal and M. M. B. Breteler, Dietary antioxidants and the risk of ischemic stroke: The Rotterdam Study, Neurology, 2003, 61, 1273-1275.

131 A. Cassidy, E. B. Rimm, É. J. O’Reilly, G. Logroscino, C. Kay, S. E. Chiuve and K. M. Rexrode, Dietary flavonoids and risk of stroke in women, Stroke, 2012, 43, 946951.

132 Z. Tang, M. Li, X. Zhang and W. Hou, Dietary flavonoid intake and the risk of stroke: A dose-response meta-analysis of prospective cohort studies, BMJ Open, 2016, 6, DOI: 10.1136/bmjopen-2015-008680.

133 P. C. H. Hollman, A. Geelen and D. Kromhout, Dietary flavonol intake may lower stroke risk in men and women, $J$. Nutr., 2010, 140, 600-604.

134 Z. M. Wang, D. Zhao, Z. L. Nie, H. Zhao, B. Zhou, W. Gao, L. S. Wang and Z. J. Yang, Flavonol intake and stroke risk: A meta-analysis of cohort studies, Nutrition, 
135 S. O. Keli, M. G. L. Hertog, E. J. M. Feskens and D. Kromhout, Dietary flavonoids, antioxidant vitamins, and incidence of stroke: The Zutphen study, Arch. Intern. Med.,

136 A. T. Hirsch and S. Duval, The global pandemic of peripheral artery disease, Lancet, 2013, 382, 1312-1314.

J. W. Olin, C. J. White, E. J. Armstrong, D. Kadian-Dodov and W. R. Hiatt, Peripheral 
Eur. J. Epidemiol., 2004, 19, 305-311.

145 M. Mohammadi-Sartang, Z. Mazloom, S. Sherafatmanesh, M. Ghorbani and D. Firoozi, Effects of supplementation with quercetin on plasma C-reactive protein concentrations: A systematic review and meta-analysis of randomized controlled trials, Eur. J. Clin. Nutr., 2017, 71, 1033-1039.

146 K. C. Maki, J. L. Slavin, T. M. Rains and P. M. Kris-Etherton, Limitations of observational evidence: Implications for evidence-based dietary recommendations, Adv. Nutr., 2014, 5, 7-15.

147 A. F. Subar, L. S. Freedman, J. A. Tooze, S. I. Kirkpatrick, C. Boushey, M. L. Neuhouser, F. E. Thompson, N. Potischman, P. M. Guenther, V. Tarasuk, J. Reedy and S. M. Krebs-Smith, Addressing Current Criticism Regarding the Value of Self-Report Dietary Data, J. Nutr., 2015, 145, 2639-2645.

148 J. R. Hébert, T. G. Hurley, S. E. Steck, D. R. Miller, F. K. Tabung, K. E. Peterson, L. H. Kushi and E. A. Frongillo, Considering the Value of Dietary Assessment Data in Informing Nutrition-Related Health Policy, Adv. Nutr., 2014, 5, 447-455.

149 N. P. Bondonno, C. P. Bondonno, N. C. Ward, J. M. Hodgson and K. D. Croft, The cardiovascular health benefits of apples: Whole fruit vs. isolated compounds, Trends Food Sci. Technol., 2017, 69, 243-256.

150 D. R. Jacobs, M. D. Gross and L. C. Tapsell, Food synergy: An operational concept for understanding nutrition, Am. J. Clin. Nutr., 2009, 89, 154S-8S.

151 R. H. Liu, Health benefits of fruit and vegetables are from additive and synergistic combinations of phytochemicals, Am. J. Clin. Nutr., 2003, 78, 517S-520S.

152 D. R. Jacobs, L. C. Tapsell and N. J. Temple, Food Synergy: The Key to Balancing the Nutrition Research Effort, Public Health Rev., 2011, 33, 507-529. 
153 P. Knekt, S. Isotupa, H. Rissanen, M. Heliövaara, R. Järvinen, S. Häkkinen, A. Aromaa and A. Reunanen, Quercetin intake and the incidence of cerebrovascular disease, Eur. J. Clin. Nutr., 2000, 54, 415-417.

154 A. Cassidy, É. J. O’Reilly, C. Kay, L. Sampson, M. Franz, J. P. Forman, G. Curhan and E. B. Rimm, Habitual intake of flavonoid subclasses and incident hypertension in adults, Am. J. Clin. Nutr., 2011, 93, 338-347.

155 G. Grosso, U. Stepaniak, A. Micek, M. Kozela, D. Stefler, M. Bobak and A. Pajak, Dietary polyphenol intake and risk of hypertension in the Polish arm of the HAPIEE study, Eur. J. Nutr., 2018, 57, 1535-1544.

156 M. Lajous, E. Rossignol, G. Fagherazzi, F. Perquier, A. Scalbert, F. Clavel-Chapelon and M. C. Boutron-Ruault, Flavonoid intake and incident hypertension in women, Am. J. Clin. Nutr., 2016, 103, 1091-1098.

157 Y. J. Liu, J. Zhan, X. L. Liu, Y. Wang, J. Ji and Q. Q. He, Dietary flavonoids intake and risk of type 2 diabetes: A meta-analysis of prospective cohort studies, Clin. Nutr., 2014, 33, 59-63.

158 M. L. Bertoia, E. B. Rimm, K. J. Mukamal, F. B. Hu, W. C. Willett and A. Cassidy, Dietary flavonoid intake and weight maintenance: Three prospective cohorts of 124 086 US men and women followed for up to 24 years, $B M J, 2016, \mathbf{3 5 2}$, DOI: 10.1136/bmj.i17.

159 L. A. E. Hughes, I. C. W. Arts, T. Ambergen, H. A. M. Brants, P. C. Dagnelie, R. A. Goldbohm, P. A. Van Den Brandt and M. P. Weijenberg, Higher dietary flavone, flavonol, and catechin intakes are associated with less of an increase in BMI over time in women: A longitudinal analysis from the Netherlands Cohort Study, Am. J. Clin. Nutr., 2008, 88, 1341-1352. 
160 F. Vieux, M. Maillot, C. D. Rehm and A. Drewnowski, Flavonoid Intakes in the US Diet Are Linked to Higher Socioeconomic Status and to Tea Consumption: Analyses of NHANES 2011-16 Data, J. Nutr., 2020, DOI: 10.1093/jn/nxaa145.

161 W. C. Willett, G. R. Howe and L. H. Kushi, Adjustment for total energy intake in epidemiologic studies, Am. J. Clin. Nutr., 1997, 65, 1220S-2338S. Patterns: Interconnections and Implications for Dietary Guidelines, Adv. Nutr., 2016, $7,445-454$.

163 P. C. Gaine, D. A. Balentine, J. W. Erdman, J. T. Dwyer, K. C. Ellwood, F. B. Hu and R. M. Russell, Are dietary bioactives ready for recommended intakes?, Adv. Nutr., 2013, 4, 539-541.

164 N. Bondonno, K. Murray, C. P. Bondonno, J. R. Lewis, K. D. Croft, C. Kyro, G. Gislason, A. Tjonneland, A. Scalbert, A. Cassidy, J. P. Piccini, K. Overvad, J. M. Hodgson and F. Dalgaard, Flavonoid intake and its association with atrial fibrillation, Clin. Nutr., 2020, DOI: doi.org/10.1016/j.clnu.2020.04.025.

165 G. Y. H. Lip, C. M. Brechin and D. A. Lane, The global burden of atrial fibrillation and stroke: A systematic review of the epidemiology of atrial fibrillation in regions outside North America and Europe, Chest, 2012, 142, 1489-1498.

166 J. R. Hébert, E. A. Frongillo, S. A. Adams, G. M. Turner-McGrievy, T. G. Hurley, D. R. Miller and I. S. Ockene, Perspective: Randomized controlled tials are not a panacea for diet-related research, Adv. Nutr., 2016, 7, 423-432.

167 A. Satija, M. J. Stampfer, E. B. Rimm, W. Willett and F. B. Hu, Perspective: Are large, simple trials the solution for nutrition research?, Adv. Nutr., 2018, 9, 378-387. 
and Multivitamin Outcomes Study, https://clinicaltrials.gov/ct2/show/NCT02422745 (accessed April 2020).

169 A. K. Illner, H. Freisling, H. Boeing, I. Huybrechts, S. P. Crispim and N. Slimani, Review and evaluation of innovative technologies for measuring diet in nutritional epidemiology, Int. J. Epidemiol., 2012, 41, 1187-1203.

170 J. P. E. Spencer, M. M. Abd El Mohsen, A. M. Minihane and J. C. Mathers, Biomarkers of the intake of dietary polyphenols: Strengths, limitations and application in nutrition research, Br. J. Nutr., 2008, 99, 12-22.

171 L. Brennan and F. B. Hu, Metabolomics-based dietary biomarkers in nutritional epidemiology—Current status and future opportunities, Mol. Nutr. Food Res., 2019, 63, DOI: $10.1002 / \mathrm{mnfr} .201701064$.

172 M. Jenab, N. Slimani, M. Bictash, P. Ferrari and S. A. Bingham, Biomarkers in nutritional epidemiology: Applications, needs and new horizons, Hum. Genet., 2009, $125,507-525$.

173 J. Rienks, J. Barbaresko and U. Nöthlings, Association of polyphenol biomarkers with cardiovascular disease and mortality risk: A systematic review and Meta-Analysis of observational studies, Nutrients, 2017, 9, 415.

174 J. Rienks, J. Barbaresko and U. Nöthlings, Association of isoflavone biomarkers with risk of chronic disease and mortality: A systematic review and meta-analysis of observational studies, Nutr. Rev., 2017, 75, 616-641.

175 N. Murphy, D. Achaintre, R. Zamora-Ros, M. Jenab, M. C. Boutron-Ruault, F. Carbonnel, I. Savoye, R. Kaaks, T. Kühn, H. Boeing, K. Aleksandrova, A. Tjønneland, C. Kyrø, K. Overvad, J. R. Quirós, M. J. Sánchez, J. M. Altzibar, J. María Huerta, A. Barricarte, K. T. Khaw, K. E. Bradbury, A. Perez-Cornago, A. Trichopoulou, A. 
Karakatsani, E. Peppa, D. Palli, S. Grioni, R. Tumino, C. Sacerdote, S. Panico, H. B. Bueno-de-Mesquita, P. H. Peeters, M. Rutegård, I. Johansson, H. Freisling, H. Noh, A. J. Cross, P. Vineis, K. Tsilidis, M. J. Gunter and A. Scalbert, A prospective evaluation of plasma polyphenol levels and colon cancer risk, Int. J. Cancer, 2018, 143, 1620 1631.

177 N. Hawkes, Sixty seconds on ... P-hacking, $B M J, 2018,362$, DOI: 10.1136/bmj.k4039.

178 M. L. Head, L. Holman, R. Lanfear, A. T. Kahn and M. D. Jennions, The extent and consequences of P-hacking in science, PLoS Biol., 2015, 13, DOI: 10.1371/journal.pbio.1002106.

179 R. Nuzzo, Scientific method: statistical errors, Nature, 2014, 506, 150-152.

180 A. Hörnell, C. Berg, E. Forsum, C. Larsson, E. Sonestedt, A. Åkesson, C. Lachat, D. Hawwash, P. Kolsteren, G. Byrnes, W. De Keyzer, J. Van Camp, J. E. Cade, D. C. Greenwood, N. Slimani, M. Cevallos, M. Egger, I. Huybrechts and E. Wirfält, Perspective: An extension of the STROBE statement for observational studies in nutritional epidemiology (STROBE-nut): Explanation and elaboration, Adv. Nutr. An Int. Rev. J., 2017, 8, 652-678.

81 D. A. Balentine, J. T. Dwyer, J. W. Erdman, M. G. Ferruzzi, P. C. Gaine, J. M. Harnly and C. L. Kwik-Uribe, Recommendations on reporting requirements for flavonoids in research, Am. J. Clin. Nutr., 2015, 202, 1113-1125. 
1792182 J. D. Wallach, K. W. Boyack and J. P. A. Ioannidis, Reproducible research practices,

1793 transparency, and open access data in the biomedical literature, 2015-2017, PLoS

1794 Biol., 2018, 16, DOI: 10.1371/journal.pbio.2006930. 